\title{
Braided Cartan Calculi and Submanifold Algebras
}

\author{
Thomas Weber* \\ Università di Napoli "FEDERICO II" and I.N.F.N. Sezione di Napoli, \\ Complesso MSA, Via Cintia, 80126 Napoli, Italy
}

January 17,2020

\begin{abstract}
We construct a noncommutative Cartan calculus on any braided commutative algebra and study its applications in noncommutative geometry. The braided Lie derivative, insertion and de Rham differential are introduced and related via graded braided commutators, also incorporating the braided Schouten-Nijenhuis bracket. The resulting braided Cartan calculus generalizes the Cartan calculus on smooth manifolds and the twisted Cartan calculus. While it is a necessity of derivation based Cartan calculi on noncommutative algebras to employ central bimodules our approach allows to consider bimodules over the full underlying algebra. Furthermore, equivariant covariant derivatives and metrics on braided commutative algebras are discussed. In particular, we prove the existence and uniqueness of an equivariant Levi-Civita covariant derivative for any fixed non-degenerate equivariant metric. Operating in a symmetric braided monoidal category we argue that Drinfel'd twist deformation corresponds to gauge equivalences of braided Cartan calculi. The notions of equivariant covariant derivative and metric are compatible with the Drinfel'd functor as well. Moreover, we project braided Cartan calculi to submanifold algebras and prove that this process commutes with twist deformation.
\end{abstract}

\section{Contents}

1 Introduction

2 Preliminaries on Quantum Groups 3

2.1 Triangular Hopf Algebras and their Representations . . . . . . . . . . . . . 3

2.2 Drinfel'd Twist Deformation . . . . . . . . . . . . . . . . 5

2.3 Equivariant Hopf Algebra Module Algebra Representations . . . . . . . . . . .

3 Braided Commutative Geometry 8

3.1 Braided Graßmann and Gerstenhaber Algebras . . . . . . . . . . . . . . . . . . . . 9

3.2 Braided Cartan Calculi and Gauge Equivalence . . . . . . . . . . . . . . . . . 14

3.3 Equivariant Covariant Derivatives and Metrics . . . . . . . . . . . . . . . . 18

4 Submanifolds in Braided Commutative Geometry 21

4.1 Braided Cartan Calculi on Submanifolds . . . . . . . . . . . . . . . . . . 21

4.2 Equivariant Covariant Derivatives on Submanifolds . . . . . . . . . . . . 22

\section{Introduction}

In 42 Stanisław Lech Woronowicz generalized the notion of Cartan calculus to quantum groups. The crucial ingredient is given by the de Rham differential, which is understood as a linear map d: $H \rightarrow \Gamma$ from a Hopf algebra $H$ to a bicovariant $H$-bimodule $\Gamma$, generated by $\mathcal{A}$ and $\mathrm{d}$, such that the Leibniz rule $\mathrm{d}(a b)=(\mathrm{d} a) b+a \mathrm{~d} b$ holds for all $a, b \in H$. It is proven that such a first order calculus admits an extension to the exterior algebra. Noncommutative calculi based on derivations rather than generalizations of differential forms are discussed by Michel Dubois-Violette, Peter Michor and Peter Schupp in [18, 19, 36, 37, though differential forms are included as dual objects to derivations. The latter approaches are suitable for general

*thomas.weber@unina.it 
noncommutative algebras in the setting of noncommutative geometry 13 . However, bimodules have to be considered over the center of the algebra. In these notes we are proposing an intermediate procedure, sticking to derivation based calculi while incorporating a Hopf algebra symmetry to avoid central bimodules. It is motivated by twisted Cartan calculi, a particular class of noncommutative Cartan calculi in the overlap of deformation quantization [8, 40, and quantum groups [21, 31. Drinfel'd twists 16] are tools to deform Hopf algebras as well as the representation theory of the Hopf algebra in a compatible way. They experienced a lot of attention in the field of deformation quantization since a Drinfel'd twist induces a star product if the corresponding symmetry acts on a smooth manifold by derivations (c.f. 3]). Explicit examples of star products are quite rare, so this connection was very desirable. However, this should be taken with a grain of salt since there are several situations [11, 15] in which deformation quantization can not be obtained via a twisting procedure. More generally, it was pointed out in [5] that a Drinfel'd twist leads to a noncommutative calculus, the so-called twisted Cartan calculus. The mentioned article even provides twisted covariant derivatives and metrics, generalizing classical Riemannian geometry. The additional braided symmetries appearing in this work were the main motivation for the author to consider noncommutative Cartan calculi only depending on a triangular structure rather than on the Drinfel'd twist itself. The appropriate categorical framework for this generalization is provided in [6, 7]: the category of equivariant braided symmetric bimodules with respect to a triangular Hopf algebra and a braided commutative algebra is symmetric braided and monoidal with respect to the tensor product over the algebra. Generalizing the algebraic construction of the Cartan calculus to this category we obtain the braided Cartan calculus. Vector fields are represented by the braided Lie algebra of braided derivations, multivector fields become a braided Gerstenhaber algebra, while differential forms constitute a braided Graßmann algebra. On the categorical level a Drinfel'd twist corresponds to a functor and its action can be understood as a gauge equivalence on the symmetric braided monoidal category (see 4, 28). We prove that this Drinfel'd functor respects the braided Cartan calculus in the sense that it intertwines the braided Lie derivative, insertion, de Rham differential and Schouten-Nijenhuis bracket. Note that both, the classical Cartan calculus and the twisted Cartan calculus, can be regarded as braided Cartan calculi. The first one with respect to any cocommutative Hopf algebra with trivial triangular structure and the latter with respect to the twisted Hopf algebra, triangular structure and algebra. In the same spirit we generalize covariant derivatives and metrics to the braided symmetric setting. Note however that for simplicity we regard them to be equivariant in addition, a requirement which excludes some interesting examples already in the twisted case. However, this assumption assures compatibility with the Drinfel'd functor. As yet another application of the braided Cartan calculus we study the braided Cartan calculus on submanifold algebras and prove that they are projected from the ambient algebra in accordance to gauge equivalences. It would be interesting to generalize the braided Cartan calculus to the setting of [6], to Lie-Rinehart algebras 27, and furthermore to connect the braided Cartan calculus to Hochschild cohomology and the Cartan calculus introduced by Boris Tsygan (see e.g. 38, 39]).

The paper is organized as follows: in Section 2 we recall basic properties of triangular Hopf algebras and study the symmetric braided monoidal category of equivariant braided symmetric bimodules of a braided commutative algebra. The Drinfel'd functor leads to a braided monoidal equivalence of this category and the one corresponding to the twisted algebra and triangular Hopf algebra. Our main result is developed in Section 3. we generalize the construction of the Cartan calculus of a commutative algebra to braided commutative algebras by incorporating a braided symmetry. Starting from the braided Lie algebra of braided derivations we build the braided Gerstenhaber algebra of braided multivector fields. The braided Schouten-Nijenhuis bracket is obtained by extending the braided commutator. The dual braided exterior algebra constitutes the braided differential forms. Then, the braided Lie derivative, insertion and de Rham differential are defined, resulting in the braided Cartan relations. In the special case of a commutative algebra we regain the commutation relations of the classical Cartan calculus. Connecting to Section 2 we introduce a twist deformation of the braided Cartan calculus and prove that it is isomorphic to the braided Cartan calculus on the twisted algebra corresponding to the twisted triangular structure. This shows that our construction respects gauge equivalence classes. As an application, we introduce equivariant covariant derivatives and metrics, give several constructions like extending them to braided multivector fields and differential forms and proving the existence and uniqueness of an equivariant Levi-Civita covariant derivative for every non-degenerate equivariant metric. The Drinfel'd functor respects the constructions. Finally in Section 4 we study braided Cartan calculi on submanifold algebras. We show how to project the algebraic structure and that this procedure commutes with twist deformation. An explicit example, given by twist quantization of quadric surfaces of $\mathbb{R}^{3}$, 
is elaborated in [25].

Throughout these notes every module is considered over a commutative ring $\mathbb{k}$. The category ${ }_{k} \mathcal{M}$ of $\mathbb{k}$-modules is monoidal with respect to the tensor product $\otimes$. If not stated otherwise every algebra is assumed to be unital and associative. A map $\Phi: V^{\bullet} \rightarrow W^{\bullet}$ between graded modules $V^{\bullet}=\bigoplus_{k \in \mathbb{Z}} V^{k}$ and $W^{\bullet}=\bigoplus_{k \in \mathbb{Z}} W^{k}$ is said to be homogeneous of degree $k \in \mathbb{Z}$ if $\Phi\left(V^{\ell}\right) \subseteq W^{k+\ell}$. We often write $\Phi: V^{\bullet} \rightarrow W^{\bullet+k}$ in this case. The graded commutator of two homogeneous maps $\Phi, \Psi: V^{\bullet} \rightarrow V^{\bullet}$ of degree $k$ and $\ell$ is defined by $[\Phi, \Psi]=\Phi \circ \Psi-(-1)^{k \ell} \Psi \circ \Phi$.

\section{Preliminaries on Quantum Groups}

In this introductory section we recall the notion of triangular Hopf algebra together with its braided monoidal category of representations. Afterwards we show how to twist the algebraic structure by a 2-cocycle and in which sense this induces an equivalence on the categorical level. In the last subsection we discuss equivariant algebra bimodules and their twist deformation. The previous braided monoidal equivalence can be refined to the bimodules which inherit a braided symmetry in addition if the algebra is braided commutative. For more details on (triangular) Hopf algebras we refer to the textbooks [12, 28, 31, 34. The more experienced readers are recommended to [4, 6, 7, 26, for a prompt discussion of what is covered in this section.

\subsection{Triangular Hopf Algebras and their Representations}

In a shortcut we introduce the category of algebras over a commutative ring $\mathbb{k}$ along with their representations. Dualizing the definition we obtain coalgebras, combining to the notion of bialgebra if the algebra and coalgebra structures respect each other. From the categorical perspective bialgebras are those algebras whose category of representations is monoidal with respect to the usual associativity and unit constraints. Integrating a braiding in this category induces universal $\mathcal{R}$-matrices on the bialgebra, while an additional antipode corresponds to a rigid (braided) monoidal category and accordingly to a (triangular) Hopf algebra on the algebraic level.

A $\mathbb{k}$-algebra is a $\mathbb{k}$-module $\mathcal{A}$ endowed with $\mathbb{k}$-linear maps $\mu: \mathcal{A} \otimes \mathcal{A} \rightarrow \mathcal{A}$ and $\eta: \mathbb{k} \rightarrow \mathcal{A}$, called product and unit of $\mathcal{A}$, such that the identities

$$
\mu \circ(\mu \otimes \mathrm{id})=\mu \circ(\mathrm{id} \otimes \mu): \mathcal{A}^{\otimes 3} \rightarrow \mathcal{A}
$$

and

$$
\mu \circ(\eta \otimes \mathrm{id})=\mathrm{id}=\mu \circ(\mathrm{id} \otimes \eta): \mathcal{A} \rightarrow \mathcal{A}
$$

hold, where we used the $\mathbb{k}$-module isomorphisms $\mathbb{k} \otimes \mathcal{A} \cong \mathcal{A} \cong \mathcal{A} \otimes \mathbb{k}$ in eq.(2.2). These are the well-known associativity and unit properties. A $\mathbb{k}$-algebra $\mathcal{A}$ is said to be commutative if $\mu_{21}=\mu$, where $\mu_{21}: \mathcal{A} \otimes \mathcal{A} \ni(a \otimes b) \mapsto \mu(b \otimes a) \in \mathcal{A}$. In the following we often drop the symbol $\mu$ and simply write $a \cdot b$ or $a b$ for the product of two elements $a, b \in \mathcal{A}$. The $\mathbb{k}$-algebras form a category ${ }_{\mathbb{k}} \mathcal{A}$ with morphisms being $\mathbb{k}$-algebra homomorphisms, i.e. $\mathbb{k}$-linear maps $\phi: \mathcal{A} \rightarrow \mathcal{A}^{\prime}$ between $\mathbb{k}$-algebras $(\mathcal{A}, \mu, \eta)$ and $\left(\mathcal{A}^{\prime}, \mu^{\prime}, \eta^{\prime}\right)$ such that

$$
\phi \circ \mu=\mu^{\prime} \circ(\phi \otimes \phi): \mathcal{A} \otimes \mathcal{A} \rightarrow \mathcal{A}^{\prime} \text { and } \phi \circ \eta=\eta^{\prime}: \mathbb{k} \rightarrow \mathcal{A}^{\prime} .
$$

Dualizing this concept we define a $\mathbb{k}$-coalgebra to be a $\mathbb{k}$-module $\mathcal{C}$ together with $\mathbb{k}$-linear maps $\Delta: \mathcal{C} \rightarrow \mathcal{C} \otimes \mathcal{C}$ and $\epsilon: \mathcal{C} \rightarrow \mathbb{k}$ satisfying

$$
(\Delta \otimes \text { id }) \circ \Delta=(\text { id } \otimes \Delta) \circ \Delta: \mathcal{C} \rightarrow \mathcal{C}^{\otimes 3}
$$

and

$$
(\epsilon \otimes \mathrm{id}) \circ \Delta=\mathrm{id}=(\mathrm{id} \otimes \epsilon) \circ \Delta: \mathcal{C} \rightarrow \mathcal{C} .
$$

The maps $\Delta$ and $\epsilon$ are said to be the coproduct and counit of $\mathcal{C}$ with the properties of being coassociative and counital, respectively. We frequently use Sweedler's sigma notation $c_{(1)} \otimes c_{(2)}$ to denote the coproduct $\Delta(c)$ of an element $c \in \mathcal{C}$, omitting a possibly finite sum of factorizing elements. By the coassociativity of $\Delta$ we further define

$$
c_{(1)} \otimes c_{(2)} \otimes c_{(3)}:=c_{(1)(1)} \otimes c_{(1)(2)} \otimes c_{(2)}=c_{(1)} \otimes c_{(2)(1)} \otimes c_{(2)(2)}
$$

and similarly for higher coproducts. A $\mathbb{k}$-coalgebra $\mathcal{C}$ is said to be cocommutative if $\Delta_{21}=\Delta$, where $\Delta_{21}(c)=c_{(2)} \otimes c_{(1)}$ for all $c \in \mathcal{C}$. A $\mathbb{k}$-coalgebra homomorphism is a $\mathbb{k}$-linear map $\psi: \mathcal{C} \rightarrow \mathcal{C}^{\prime}$ between $\mathbb{k}$-coalgebras $(\mathcal{C}, \Delta, \epsilon)$ and $\left(\mathcal{C}^{\prime}, \Delta^{\prime}, \epsilon^{\prime}\right)$ obeying the relations

$$
\Delta^{\prime} \circ \psi=(\psi \otimes \psi) \circ \Delta: \mathcal{C} \rightarrow \mathcal{C}^{\prime} \otimes \mathcal{C}^{\prime} \text { and } \epsilon^{\prime} \circ \psi=\epsilon: \mathcal{C} \rightarrow \mathbb{k} .
$$

The category of $\mathbb{k}$-comodules is denoted by ${ }_{\mathbb{k}} \mathcal{C}$. 
Example 2.1. We give some elementary examples and constructions of (co)algebras, focusing on the ones we need in the rest of these notes.

i.) The tensor product $\mathcal{A} \otimes \mathcal{A}^{\prime}$ of two $\mathbb{k}$-algebras $(\mathcal{A}, \mu, \eta)$ and $\left(\mathcal{A}^{\prime}, \mu^{\prime}, \eta^{\prime}\right)$ becomes a $\mathbb{k}$-algebra with product

$$
\mu_{\mathcal{A} \otimes \mathcal{A}^{\prime}}=\left(\mu \otimes \mu^{\prime}\right) \circ\left(\mathrm{id} \otimes \tau_{\mathcal{A}^{\prime}, \mathcal{A}} \otimes \mathrm{id}\right):\left(\mathcal{A} \otimes \mathcal{A}^{\prime}\right) \otimes\left(\mathcal{A} \otimes \mathcal{A}^{\prime}\right) \rightarrow \mathcal{A} \otimes \mathcal{A}^{\prime}
$$

and unit $\eta_{\mathcal{A} \otimes \mathcal{A}^{\prime}}=\eta \otimes \eta^{\prime}$, where we use the $\mathbb{k}$-module isomorphism $\mathbb{k} \otimes \mathbb{k} \cong \mathbb{k}$ in the latter definition and $\tau_{\mathcal{A}^{\prime}, \mathcal{A}}: \mathcal{A}^{\prime} \otimes \mathcal{A} \rightarrow \mathcal{A} \otimes \mathcal{A}^{\prime}$ denotes the tensor flip isomorphism. Dually, the tensor product $\mathcal{C} \otimes \mathcal{C}^{\prime}$ of two $\mathbb{k}$-coalgebras $(\mathcal{C}, \Delta, \epsilon)$ and $\left(\mathcal{C}^{\prime}, \Delta^{\prime}, \epsilon^{\prime}\right)$ can be structured as a $\mathbb{k}$-coalgebra with coproduct

$$
\Delta_{\mathcal{C} \otimes \mathcal{C}^{\prime}}=\left(\mathrm{id} \otimes \tau_{\mathcal{C}, \mathcal{C}^{\prime}} \otimes \mathrm{id}\right) \circ\left(\Delta \otimes \Delta^{\prime}\right): \mathcal{C} \otimes \mathcal{C}^{\prime} \rightarrow\left(\mathcal{C} \otimes \mathcal{C}^{\prime}\right) \otimes\left(\mathcal{C} \otimes \mathcal{C}^{\prime}\right)
$$

and counit $\epsilon_{\mathcal{C} \otimes \mathcal{C}^{\prime}}=\epsilon \otimes \epsilon^{\prime}$.

ii.) Any commutative ring $\mathbb{k}$ is a $\mathbb{k}$-(co)algebra with product and unit given by its ring multiplication and unit element, while the coproduct and counit are defined by $\Delta(\lambda)=\lambda(1 \otimes 1)$ and $\epsilon(\lambda)=\lambda$ for all $\lambda \in \mathbb{k}$.

A $\mathbb{k}$-algebra $(\mathcal{A}, \mu, \eta)$ which is also a $\mathbb{k}$-coalgebra with coproduct $\Delta$ and counit $\epsilon$ is said to be a $\mathbb{k}$-bialgebra if $\Delta$ and $\epsilon$ are $\mathbb{k}$-algebra homomorphisms and $\mu$ and $\eta$ are $\mathbb{k}$-coalgebra homomorphisms. It is clear by the symmetry in the definition of algebra and coalgebra that a $\mathbb{k}$-algebra and $\mathbb{k}$-coalgebra is a $\mathbb{k}$-bialgebra if and only if its algebra structures are $\mathbb{k}$-coalgebra homomorphisms if and only if its coalgebra structures are $\mathbb{k}$-algebra homomorphisms. A $\mathbb{k}$ bialgebra homomorphism is a $\mathbb{k}$-algebra homomorphism between $\mathbb{k}$-bialgebras which is also a $\mathbb{k}$-coalgebra homomorphism.

Definition 2.2. A $\mathbb{k}$-bialgebra $(H, \mu, \eta, \Delta, \epsilon)$ is said to be triangular if there is an invertible element $\mathcal{R} \in H \otimes H$, called universal $\mathcal{R}$-matrix or triangular structure, with inverse given by $\mathcal{R}_{21}=\tau_{H, H}(\mathcal{R})$, such that

$$
\Delta_{21}(\xi)=\mathcal{R} \Delta(\xi) \mathcal{R}^{-1} \text { for all } \xi \in H,
$$

and the hexagon relations

$$
(\Delta \otimes \mathrm{id})(\mathcal{R})=\mathcal{R}_{13} \mathcal{R}_{23} \text { and }(\mathrm{id} \otimes \Delta)(\mathcal{R})=\mathcal{R}_{13} \mathcal{R}_{12}
$$

are satisfied, where $\mathcal{R}_{12}=\mathcal{R} \otimes 1, \mathcal{R}_{23}=1 \otimes \mathcal{R}, \mathcal{R}_{13}=\left(\mathrm{id} \otimes \tau_{H, H}\right)\left(\mathcal{R}_{12}\right) \in H^{\otimes 3}$. Property (2.8) states that $H$ is quasi-cocommutative. The $\mathbb{k}$-bialgebra $H$ is said to be a $\mathbb{k}$-Hopf algebra if there is a bijective $\mathbb{k}$-linear map $S: H \rightarrow H$, called antipode, such that

$$
\mu \circ(S \otimes \mathrm{id}) \circ \Delta=\eta \circ \epsilon=\mu \circ(\mathrm{id} \otimes S) \circ \Delta: H \rightarrow H
$$

holds. A $\mathbb{k}$-bialgebra homomorphism between $\mathbb{k}$-Hopf algebras is said to be a $\mathbb{k}$-Hopf algebra homomorphism if it intertwines the antipodes. We denote the category of $\mathbb{k}$-Hopf algebras by ${ }_{\mathbb{k}} \mathcal{H}$. A $\mathbb{k}$-Hopf algebra $(H, \mu, \eta, \Delta, \epsilon, S)$ is called triangular if its underlying bialgebra structure is.

In the following we often drop the reference to the commutative ring $\mathbb{k}$ and simply refer to Hopf algebras etc. Remark that there are slightly weaker definitions of Hopf algebra, not assuming the antipode to have an inverse (see [28, 31, 34]). We follow the convention of [12], arguing that in all examples which are relevant for us the antipode is invertible and we do not want to state this as an additional condition throughout. One can show that the antipode $S$ of a bialgebra $(H, \mu, \eta, \Delta, \epsilon)$ is unique if it exists and that it is an anti-bialgebra homomorphism in the sense that

$$
S(\xi \chi)=S(\chi) S(\xi), S(1)=1, S(\xi)_{(1)} \otimes S(\xi)_{(2)}=S\left(\xi_{(2)}\right) \otimes S\left(\xi_{(1)}\right) \text { and } \epsilon \circ S=\epsilon
$$

for all $\xi, \chi \in H$. If $H$ is commutative or cocommutative it follows that $S^{2}=$ id. Moreover, any cocommutative Hopf algebra is triangular with universal $\mathcal{R}$-matrix given by $\mathcal{R}=1 \otimes 1$. Any universal $\mathcal{R}$-matrix $\mathcal{R}$ satisfies the quantum Yang-Baxter equation

$$
\mathcal{R}_{12} \mathcal{R}_{13} \mathcal{R}_{23}=\mathcal{R}_{23} \mathcal{R}_{13} \mathcal{R}_{12}
$$

Fix a triangular $\mathbb{k}$-bialgebra $(H, \mu, \eta, \Delta, \epsilon, \mathcal{R})$ for the moment. We motivate its definition by elaborating that the representation theory of $H$ has interesting categorical properties. Recall that a representation of $H$ is nothing but a left $H$-module, i.e. a $\mathbb{k}$-module $\mathcal{M}$ together with a $\mathbb{k}$-linear map $\lambda: H \otimes \mathcal{M} \rightarrow \mathcal{M}$, called left $H$-module action or left $H$-module structure, such that

$$
\lambda \circ\left(\operatorname{id}_{H} \otimes \lambda\right)=\lambda \circ\left(\mu \otimes \operatorname{id}_{\mathcal{M}}\right): H \otimes H \otimes \mathcal{M} \rightarrow \mathcal{M}
$$


and $\lambda \circ\left(\eta \otimes \operatorname{id}_{\mathcal{M}}\right)=\operatorname{id}_{\mathcal{M}}$ hold. A left $H$-module homomorphism is a $\mathbb{k}$-linear map $\Phi: \mathcal{M} \rightarrow \mathcal{M}^{\prime}$ between left $H$-modules $(\mathcal{M}, \lambda)$ and $\left(\mathcal{M}^{\prime}, \lambda^{\prime}\right)$ such that

$$
\Phi \circ \lambda=\lambda^{\prime} \circ\left(\operatorname{id}_{H} \otimes \Phi\right): H \otimes \mathcal{M} \rightarrow \mathcal{M}^{\prime} .
$$

We sometimes refer to left $H$-module homomorphisms as $H$-equivariant maps. This forms the category ${ }_{H} \mathcal{M}$ of left $H$-modules. In the following we often write $\xi \cdot m$ instead of $\lambda(\xi \otimes m)$ for a left $H$-module $(\mathcal{M}, \lambda)$, where $\xi \in H$ and $m \in \mathcal{M}$. Note that until now we only used the algebra structure of $H$ in the definition of ${ }_{H} \mathcal{M}$. In other words, we can consider the category of representations for any algebra. However, since $\Delta$ and $\epsilon$ are algebra homomorphisms we can define a left $H$-module action on the tensor product of two left $H$-modules $(\mathcal{M}, \lambda)$ and $\left(\mathcal{M}^{\prime}, \lambda^{\prime}\right)$ by

$$
\lambda_{\mathcal{M} \otimes \mathcal{M}^{\prime}}=\left(\lambda \otimes \lambda^{\prime}\right) \circ\left(\operatorname{id}_{H} \otimes \tau_{H, \mathcal{M}} \otimes \operatorname{id}_{\mathcal{M}^{\prime}}\right) \circ\left(\Delta \otimes \operatorname{id}_{\mathcal{M} \otimes \mathcal{M}^{\prime}}\right): H \otimes\left(\mathcal{M} \otimes \mathcal{M}^{\prime}\right) \rightarrow \mathcal{M} \otimes \mathcal{M}^{\prime}
$$

and a left $H$-module action on $\mathbb{k}$ by

$$
\lambda_{\mathbb{k}}=\left(\epsilon \otimes \operatorname{id}_{\mathbb{k}}\right): H \otimes \mathbb{k} \rightarrow \mathbb{k} \otimes \mathbb{k} \cong \mathbb{k} .
$$

Those actions respect the usual associativity and unit constraints of the tensor product of $\mathbb{k}$ modules because $\Delta$ is coassociative and $\epsilon$ satisfies the counit axiom. In other words, $(H \mathcal{M}, \otimes)$ is a monoidal category. The universal $\mathcal{R}$-matrix $\mathcal{R}$ induces a symmetric braiding on this category by defining

$$
c_{\mathcal{M}, \mathcal{M}^{\prime}}^{\mathcal{R}}\left(m \otimes m^{\prime}\right)=\mathcal{R}^{-1} \cdot\left(m^{\prime} \otimes m\right) \in \mathcal{M}^{\prime} \otimes \mathcal{M} \text { for all } m \in \mathcal{M}, m^{\prime} \in \mathcal{M}^{\prime} .
$$

In fact, the hexagon relations of $\mathcal{R}$ correspond to the hexagon relations of $c^{\mathcal{R}}$ and $c_{\mathcal{M}, \mathcal{M}^{\prime}}^{\mathcal{R}} \circ$ $c_{\mathcal{M}^{\prime}, \mathcal{M}}^{\mathcal{R}}=\operatorname{id}_{\mathcal{M}^{\prime} \otimes \mathcal{M}}$ since $\mathcal{R}_{21}$ is the inverse of $\mathcal{R}$. Conversely, any symmetric braiding $c$ on $\left({ }_{H} \mathcal{M}, \otimes\right)$ determines a triangular structure $\mathcal{R}=\tau_{H, H}\left(c_{H, H}(1 \otimes 1)\right) \in H \otimes H$, where $H$ acts on itself by left multiplication.

Proposition 2.3 (28 Proposition XIII.1.4.). The representation theory ${ }_{H} \mathcal{M}$ of a $\mathbb{k}$-bialgebra is a monoidal category. It is braided symmetric if and only if $H$ is triangular.

In the case of a Hopf algebra $(H, \mu, \eta, \Delta, \epsilon, S)$ we receive an additional rigidity property of its monoidal category in the sense that every left $H$-module admits a left and right dual module. However, for this we have to restrict our consideration to finitely generated projective $\mathbb{k}$-modules ${ }_{\mathbb{k}} \mathcal{M}^{f}$. The antipode of $H$ can be used to transfer the rigidity property from ${ }_{\mathbb{k}} \mathcal{M}^{f}$ to ${ }_{H} \mathcal{M}^{f}$. Denote the usual dual pairing of a finitely generated projective $\mathbb{k}$-module $\mathcal{M}$ and its dual module $\mathcal{M}^{*}$ by $\langle\cdot, \cdot\rangle: \mathcal{M}^{*} \otimes \mathcal{M} \rightarrow \mathbb{k}$.

Proposition 2.4 (12 Example 5.1.4). Let $H$ be a $\mathbb{k}-H o p f$ algebra and consider the monoidal category ${ }_{\mathrm{H}} \mathcal{M}$ of left $H$-modules. The monoidal subcategory ${ }_{H} \mathcal{M}^{f}$ of finitely generated projective left $H$-modules is rigid, where the left and right dual $\mathcal{M}^{*}$ and ${ }^{*} \mathcal{M}$ of an object $\mathcal{M}$ in ${ }_{H} \mathcal{M}^{f}$ are defined as the finitely generated projective $\mathbb{k}$-module $\mathcal{M}^{*}$ with left $H$-module action given by

$$
\langle\xi \cdot \alpha, m\rangle=\langle\alpha, S(\xi) \cdot m\rangle
$$

and

$$
\langle\xi \cdot \alpha, m\rangle=\left\langle\alpha, S^{-1}(\xi) \cdot m\right\rangle
$$

for all $\xi \in H, m \in \mathcal{M}$ and $\alpha \in \mathcal{M}^{*}$, respectively. The forgetful functor

$$
F:{ }_{H} \mathcal{M}^{f} \rightarrow{ }_{\mathbb{k}} \mathcal{M}^{f}
$$

is monoidal.

\subsection{Drinfel'd Twist Deformation}

In this subsection we introduce Drinfel'd twists as an instrument to deform (triangular) Hopf algebra structures. It turns out that the representation theory of the deformed (triangular) Hopf algebra is (braided) monoidally equivalent the representation theory of the undeformed (triangular) Hopf algebra. The definition of Drinfel'd twist originates from [16, while the monoidal equivalence was proven in [17. We further refer to 4, 26, for a discussion of this topic. Fix a Hopf algebra $(H, \mu, \eta, \Delta, \epsilon, S)$ in the following.

Definition 2.5. A (Drinfel'd) twist on $H$ is an invertible element $\mathcal{F} \in H \otimes H$ satisfying the 2-cocycle condition

$$
(\mathcal{F} \otimes 1)(\Delta \otimes \mathrm{id})(\mathcal{F})=(1 \otimes \mathcal{F})(\mathrm{id} \otimes \Delta)(\mathcal{F})
$$

and the normalization condition $(\epsilon \otimes \mathrm{id})(\mathcal{F})=1=(\mathrm{id} \otimes \epsilon)(\mathcal{F})$. 
There are several examples and constructions of Drinfel'd twists, showing that this is a rich concept. We refer the interested reader to [20, 33. It follows that the inverse $\mathcal{F}^{-1}$ of a twist $\mathcal{F}$ on $H$ is normalized, i.e. $(\epsilon \otimes \mathrm{id})\left(\mathcal{F}^{-1}\right)=1=(\mathrm{id} \otimes \epsilon)\left(\mathcal{F}^{-1}\right)$ and satisfies the so called inverse 2-cocycle condition

$$
(\Delta \otimes \mathrm{id})\left(\mathcal{F}^{-1}\right)\left(\mathcal{F}^{-1} \otimes 1\right)=(\mathrm{id} \otimes \Delta)\left(\mathcal{F}^{-1}\right)\left(1 \otimes \mathcal{F}^{-1}\right) .
$$

Any element $\mathcal{F} \in H \otimes H$ can be written as a finite sum of factorizing elements $\mathcal{F}_{1}^{i} \otimes \mathcal{F}_{2}^{i}$, $\mathcal{F}_{1}^{i}, \mathcal{F}_{2}^{i} \in H$. In the following we usually omit this finite sum and simply write $\mathcal{F}=\mathcal{F}_{1} \otimes \mathcal{F}$, which is called leg notation. Using this convention, the 2-cocycle (2.16) condition reads

$$
\mathcal{F}_{1} \mathcal{F}_{1(1)}^{\prime} \otimes \mathcal{F}_{2} \mathcal{F}_{1(2)}^{\prime} \otimes \mathcal{F}_{2}^{\prime}=\mathcal{F}_{1}^{\prime} \otimes \mathcal{F}_{1} \mathcal{F}_{2(1)}^{\prime} \otimes \mathcal{F}_{2} \mathcal{F}_{2(2)}^{\prime},
$$

where we marked the second copy of $\mathcal{F}$ by $\mathcal{F}=\mathcal{F}_{1}^{\prime} \otimes \mathcal{F}_{2}^{\prime}$ to distinguish the summations. The following proposition (c.f. 31] Theorem 2.3.4) reveals the utility of Drinfel'd twists as they provide a construction of (triangular) Hopf algebras from given ones.

Proposition 2.6. Consider a twist $\mathcal{F}$ on $H$. Then $H_{\mathcal{F}}=\left(H, \mu, \eta, \Delta_{\mathcal{F}}, \epsilon, S_{\mathcal{F}}\right)$ is a Hopf algebra with coproduct and antipode given by

$$
\Delta_{\mathcal{F}}(\xi)=\mathcal{F} \Delta(\xi) \mathcal{F}^{-1} \text { and } S_{\mathcal{F}}(\xi)=\beta S(\xi) \beta^{-1},
$$

respectively, for all $\xi \in H$, where $\beta=\mathcal{F}_{1} S\left(\mathcal{F}_{2}\right) \in H$. If $H$ is triangular with universal $\mathcal{R}$-matrix $\mathcal{R}$, so is $H_{\mathcal{F}}$ with universal $\mathcal{R}$-matrix $\mathcal{R}_{\mathcal{F}}=\mathcal{F}_{21} \mathcal{R F}^{-1}$.

Let $\mathcal{F}$ be a twist on $H$ and consider the corresponding monoidal category $\left(H_{\mathcal{F}} \mathcal{M}, \otimes_{\mathcal{F}}\right)$ of representations of $H_{\mathcal{F}}$. Since $H$ and $H_{\mathcal{F}}$ coincide as algebras every left $H$-module is automati-

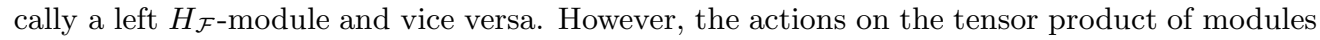
differ in general. For this reason we denote the monoidal structure of $H_{\mathcal{F}} \mathcal{M}$ by $\otimes_{\mathcal{F}}$. Namely, for two left $H$-modules (or equivalently two left $H_{\mathcal{F}}$-modules) $\mathcal{M}$ and $\mathcal{M}^{\prime}$ the tensor product $\mathcal{M} \otimes_{\mathcal{F}} \mathcal{M}^{\prime}$ coincides with $\mathcal{M} \otimes \mathcal{M}^{\prime}$ as a $\mathbb{k}$-module but $\mathcal{M} \otimes_{\mathcal{F}} \mathcal{M}^{\prime}$ is a left $H_{\mathcal{F} \text {-module via }}$

$$
\xi \cdot\left(m \otimes_{\mathcal{F}} m^{\prime}\right)=\left(\xi_{\widehat{(1)}} \cdot m\right) \otimes_{\mathcal{F}}\left(\xi_{\widehat{(2)}} \cdot m^{\prime}\right),
$$

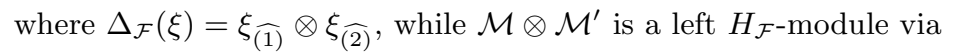

$$
\xi \cdot\left(m \otimes m^{\prime}\right)=\left(\xi_{(1)} \cdot m\right) \otimes\left(\xi_{(2)} \cdot m^{\prime}\right)
$$

for all $\xi \in H_{\mathcal{F}}, m \in \mathcal{M}$ and $m^{\prime} \in \mathcal{M}^{\prime}$. We are able to compare those pictures via a left $H_{\mathcal{F}}$-module isomorphism

$$
\varphi_{\mathcal{M}, \mathcal{M}^{\prime}}: \mathcal{M} \otimes_{\mathcal{F}} \mathcal{M}^{\prime} \ni\left(m \otimes \mathcal{F} m^{\prime}\right) \mapsto\left(\mathcal{F}_{1}^{-1} \cdot m\right) \otimes\left(\mathcal{F}_{2}^{-1} \cdot m^{\prime}\right) \in \mathcal{M} \otimes \mathcal{M}^{\prime}
$$

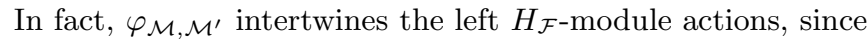

$$
\varphi_{\mathcal{M}, \mathcal{M}^{\prime}}\left(\xi \cdot\left(m \otimes_{\mathcal{F}} m^{\prime}\right)\right)=\left(\left(\xi_{(1)} \mathcal{F}_{1}^{-1}\right) \cdot m\right) \otimes\left(\left(\xi_{(2)} \mathcal{F}_{2}^{-1}\right) \cdot m\right)=\xi \cdot \varphi_{\mathcal{M}, \mathcal{M}^{\prime}}\left(m \otimes \mathcal{F} m^{\prime}\right)
$$

for all $\xi \in H, m \in \mathcal{M}$ and $m^{\prime} \in \mathcal{M}^{\prime}$ and admits an inverse left $H_{\mathcal{F} \text {-module homomorphism }}$

$$
\varphi_{\mathcal{M}, \mathcal{M}^{\prime}}^{-1}: \mathcal{M} \otimes \mathcal{M}^{\prime} \ni\left(m \otimes m^{\prime}\right) \mapsto\left(\mathcal{F}_{1} \cdot m\right) \otimes_{\mathcal{F}}\left(\mathcal{F}_{2} \cdot m^{\prime}\right) \in \mathcal{M} \otimes_{\mathcal{F}} \mathcal{M}^{\prime}
$$

The map $\varphi$ gives rise to a monoidal equivalence. We formulate this in the following theorem (c.f. 28] Lemma XV.3.7.).

Theorem 2.7. For any twist $\mathcal{F}$ on $H$ there is a monoidal equivalence of the monoidal categories $(H \mathcal{M}, \otimes)$ and $\left(H_{\mathcal{F}} \mathcal{M}, \otimes_{\mathcal{F}}\right)$. If $H$ is triangular we obtain a braided monoidal equivalence between braided monoidal categories $\left(H \mathcal{M}, \otimes, c^{\mathcal{R}}\right)$ and $\left(H_{\mathcal{F}} \mathcal{M}, \otimes_{\mathcal{F}}, c^{\mathcal{R}_{\mathcal{F}}}\right)$.

In the light of this theorem Drinfel'd twists are sometimes referred to as gauge transformations or gauge equivalences (see e.g. 28. Section XV.3). This nomenclature is affirmed by the observation that $1 \otimes 1 \in H \otimes H$ is a Drinfel'd twist on any Hopf algebra $H$ and if $\mathcal{F}$ is a twist on $H$ and $\mathcal{F}^{\prime}$ a twist on $H_{\mathcal{F}}$, the product $\mathcal{F}^{\prime} \mathcal{F}$ is a Drinfel'd twist on $H$ such that $H_{\mathcal{F}^{\prime} \mathcal{F}}=\left(H_{\mathcal{F}}\right)_{\mathcal{F}^{\prime}}$. 


\subsection{Equivariant Hopf Algebra Module Algebra Representations}

For some applications the monoidal equivalence ${ }_{H} \mathcal{M} \cong{ }_{H_{\mathcal{F}}} \mathcal{M}$ of Theorem 2.7] is too arbitrary. Motivated from differential geometry we want to study equivariant module algebra bimodules instead, which generalize equivariant vector bundles. However, the restriction of the monoidal equivalence to those bimodules fails to be braided in general. To fix this we have to restrict ourselves to braided commutative algebras and equivariant braided symmetric algebra bimodules. Nonetheless, this setting is rich enough to allow for several interesting examples, e.g. the braided multivector fields and differential forms of a braided commutative algebra, as we see in Sections 3.1

Fix a Hopf algebra $(H, \mu, \eta, \Delta, \epsilon, S)$ and consider a left $H$-module $(\mathcal{A}, \lambda)$ which is an algebra with product $\mu_{\mathcal{A}}$ and unit $\eta_{\mathcal{A}}$ in addition. It is said to be a left $H$-module algebra if the module action respects the algebra structure, i.e. if

$$
\lambda \circ\left(\operatorname{id}_{H} \otimes \mu_{\mathcal{A}}\right)=\mu_{\mathcal{A}} \circ(\lambda \otimes \lambda) \circ\left(\operatorname{id}_{H} \otimes \tau_{H, \mathcal{A}} \otimes \operatorname{id}_{\mathcal{A}}\right) \circ\left(\Delta \otimes \operatorname{id}_{\mathcal{A} \otimes \mathcal{A}}\right): H \otimes \mathcal{A} \otimes \mathcal{A} \rightarrow \mathcal{A}
$$

and $\lambda \circ\left(\operatorname{id}_{H} \otimes \eta_{\mathcal{A}}\right)=\eta_{\mathcal{A}} \circ \epsilon: H \rightarrow \mathcal{A}$ hold. In the following we often write $\mu_{\mathcal{A}}(a \otimes b)=a \cdot b$ for $a, b \in \mathcal{A}$ and $\xi \triangleright a$ for the module action of $\xi \in H$ on $a \in \mathcal{A}$. The units of $\mathcal{A}$ and $H$ are sometimes denoted by $1_{\mathcal{A}}$ and $1_{H}$, respectively or simply by 1 . In this notation the module algebra axioms read

$$
\xi \triangleright(a \cdot b)=\left(\xi_{(1)} \triangleright a\right) \cdot\left(\xi_{(2)} \triangleright b\right) \text { and } \xi \triangleright 1_{\mathcal{A}}=\epsilon(\xi) 1_{\mathcal{A}}
$$

for all $\xi \in H$ and $a, b \in \mathcal{A}$. A left $H$-module algebra homomorphism is a left $H$-module homomorphism between left $H$-module algebras which is also an algebra homomorphism. The category of left $H$-module algebras is denoted by ${ }_{H} \mathcal{A}$.

Lemma 2.8 (4 Theorem 3.4). Let $\mathcal{F}$ be a twist on $H$ and consider a left $H$-module algebra $\left(\mathcal{A}, \cdot 1_{\mathcal{A}}\right)$. Then $\mathcal{A}_{\mathcal{F}}=\left(\mathcal{A}, \cdot_{\mathcal{F}}, 1_{\mathcal{A}}\right)$ is a left $H_{\mathcal{F}}$-module algebra with respect to the same left $H$-module action, where

$$
a \cdot \mathcal{F} b=\left(\mathcal{F}_{1}^{-1} \triangleright a\right) \cdot\left(\mathcal{F}_{2}^{-1} \triangleright b\right)
$$

for all $a, b \in \mathcal{A}$.

Fix a left $H$-module algebra $\mathcal{A}$ in the following and consider the category ${ }_{\mathcal{A}} \mathcal{M}$ of left $\mathcal{A}$ modules. In order to compare it to the representation theory of the deformed algebra $\mathcal{A}_{\mathcal{F}}$ we have to incorporate an additional action of the Hopf algebra $H$ on the modules. To obtain interesting results this action has to respect the $\mathcal{A}$-module structure. Accordingly we consider the subcategory ${ }_{\mathcal{A}}^{H} \mathcal{M}$ of $H$-equivariant left $\mathcal{A}$-modules. The objects of ${ }_{\mathcal{A}}^{H} \mathcal{M}$ are left $H$-modules $\mathcal{M}$, which are left $\mathcal{A}$-modules in addition such that

$$
\xi \triangleright(a \cdot m)=\left(\xi_{(1)} \triangleright a\right) \cdot\left(\xi_{(2)} \triangleright m\right)
$$

for all $\xi \in H, a \in \mathcal{A}$ and $m \in \mathcal{M}$. Morphisms are left $H$-module homomorphisms between $H$-equivariant left $\mathcal{A}$-modules which are also left $\mathcal{A}$-module homomorphisms.

Lemma 2.9. Let $\mathcal{F}$ be a twist on $H$ and $\mathcal{A}$ a left $H$-module algebra. Then there is a functor

$$
\operatorname{Drin}_{\mathcal{F}}:{ }_{\mathcal{A}}^{H} \mathcal{M} \rightarrow{ }_{\mathcal{A}_{\mathcal{F}}}^{H_{\mathcal{F}}} \mathcal{M},
$$

called Drinfel'd functor, which is the identity on morphisms and assigns to every $H$-equivariant left $\mathcal{A}$-module $\mathcal{M}$ the same left $H$-module but with left $\mathcal{A}_{\mathcal{F}}$-module structure given by

$$
a \cdot \mathcal{F} m=\left(\mathcal{F}_{1}^{-1} \triangleright a\right) \cdot\left(\mathcal{F}_{2}^{-1} \triangleright m\right)
$$

for all $a \in \mathcal{A}$ and $m \in \mathcal{M}$.

Proof. In fact, the obtained $\mathbb{k}$-module $\mathcal{M}_{\mathcal{F}}$ is an object in ${ }_{\mathcal{A}_{\mathcal{F}}}^{H_{\mathcal{F}}} \mathcal{M}$, since

$$
(a \cdot \mathcal{F} b) \cdot \mathcal{F} m=a \cdot \mathcal{F}\left(b \cdot_{\mathcal{F}} m\right) \text { and } \xi \triangleright\left(a \cdot_{\mathcal{F}} m\right)=\left(\xi_{\widehat{(1)}} \triangleright a\right) \cdot_{\mathcal{F}}\left(\xi_{\widehat{(2)}} \triangleright m\right)
$$

follow for all $\xi \in H, a, b \in \mathcal{A}$ and $m \in \mathcal{M}$ in complete analogy to Lemma 2.8. Furthermore, any morphisms $\phi: \mathcal{M} \rightarrow \mathcal{M}^{\prime}$ in ${ }_{\mathcal{A}}^{H} \mathcal{M}$ is automatically a morphism in ${ }_{\mathcal{A}_{\mathcal{F}}}^{H_{\mathcal{F}}} \mathcal{M}$, where left $H_{\mathcal{F}}$-linearity is trivially given and left $\mathcal{A}_{\mathcal{F}}$-linearity follows since

$$
\begin{aligned}
\phi(a \cdot \mathcal{F} m) & =\phi\left(\left(\mathcal{F}_{1}^{-1} \triangleright a\right) \cdot\left(\mathcal{F}_{2}^{-1} \triangleright m\right)\right)=\left(\mathcal{F}_{1}^{-1} \triangleright a\right) \cdot \phi\left(\mathcal{F}_{2}^{-1} \triangleright m\right) \\
& =\left(\mathcal{F}_{1}^{-1} \triangleright a\right) \cdot\left(\mathcal{F}_{2}^{-1} \triangleright \phi(m)\right)=a \cdot \mathcal{F} \phi(b)
\end{aligned}
$$

for all $a \in \mathcal{A}$ and $m \in \mathcal{M}$. 
One might ask if the monoidal equivalence of Theorem 2.7 restricts to ${ }_{\mathcal{A}}^{H} \mathcal{M}$. However, ${ }_{\mathcal{A}}^{H} \mathcal{M}$ is not monoidal with respect to the usual tensor product of $\mathbb{k}$-modules, since there is no coproduct on $\mathcal{A}$ in general to distribute the left $\mathcal{A}$-module action to the tensor factors. To obtain a monoidal category we need two specifications: first we consider the subcategory of $H$-equivariant $\mathcal{A}$-bimodules ${ }_{\mathcal{A}}^{H} \mathcal{M}_{\mathcal{A}}$, i.e. there are commuting left and right $\mathcal{A}$-actions which are equivariant with respect to the left $H$-action. Secondly, we consider the tensor product $\otimes_{\mathcal{A}}$ over $\mathcal{A}$, which is defined for two objects $\mathcal{M}$ and $\mathcal{M}^{\prime}$ by the quotient

$$
\mathcal{M} \otimes \mathcal{M}^{\prime} / N_{\mathcal{M}, \mathcal{M}^{\prime}}
$$

where $N_{\mathcal{M}, \mathcal{M}}=\operatorname{im}\left(\rho_{\mathcal{M}} \otimes \operatorname{id}_{\mathcal{M}^{\prime}}-\operatorname{id}_{\mathcal{M}} \otimes \lambda_{\mathcal{M}^{\prime}}\right)$ and $\lambda_{\mathcal{M}^{\prime}}$ and $\rho_{\mathcal{M}}$ denote the left and right $\mathcal{A}$-actions on $\mathcal{M}^{\prime}$ and $\mathcal{M}$, respectively. As a consequence one has

$$
(m \cdot a) \otimes_{\mathcal{A}} m^{\prime}=m \otimes_{\mathcal{A}}\left(a \cdot m^{\prime}\right)
$$

for all $a \in \mathcal{A}, m \in \mathcal{M}$ and $m^{\prime} \in \mathcal{M}^{\prime}$. Then $\mathcal{M} \otimes_{\mathcal{A}} \mathcal{M}^{\prime}$ is an $H$-equivariant $\mathcal{A}$-bimodule, with induced left $H$-action and left and right $\mathcal{A}$-actions given by

$$
a \cdot\left(m \otimes_{\mathcal{A}} m^{\prime}\right)=(a \cdot m) \otimes_{\mathcal{A}} m^{\prime} \text { and }\left(m \otimes_{\mathcal{A}} m^{\prime}\right) \cdot a=m \otimes_{\mathcal{A}}\left(m^{\prime} \cdot a\right)
$$

for all $a \in \mathcal{A}, m \in \mathcal{M}$ and $m^{\prime} \in \mathcal{M}^{\prime}$. On morphisms $\phi: \mathcal{M} \rightarrow \mathcal{N}$ and $\psi: \mathcal{M}^{\prime} \rightarrow \mathcal{N}^{\prime}$ of ${ }_{\mathcal{A}}^{H} \mathcal{M}_{\mathcal{A}}$ one defines $\left(\phi \otimes_{\mathcal{A}} \psi\right)\left(m \otimes_{\mathcal{A}} m^{\prime}\right)=\phi(m) \otimes_{\mathcal{A}} \psi\left(m^{\prime}\right)$ for all $m \in \mathcal{M}$ and $m^{\prime} \in \mathcal{M}^{\prime}$.

Proposition 2.10. The tuple $\left({ }_{\mathcal{A}}^{H} \mathcal{M}_{\mathcal{A}}, \otimes_{\mathcal{A}}\right)$ is a monoidal category and for a twist $\mathcal{F}$ on $H$ the monoidal equivalence of Theorem 2.7 descends to a monoidal equivalence of $\left({ }_{\mathcal{A}}^{H} \mathcal{M}_{\mathcal{A}}, \otimes_{\mathcal{A}}\right)$ and $\left({ }_{\mathcal{A}_{\mathcal{F}}}^{H_{\mathcal{F}}} \mathcal{M}_{\mathcal{A}_{\mathcal{F}}}, \otimes_{\mathcal{A}_{\mathcal{F}}}\right)$.

We refer to 6 Theorem 3.13 for a proof and more information. In contrast to Theorem 2.7 we do not obtain a symmetric braided monoidal structure on ${ }_{\mathcal{A}}^{H} \mathcal{M}_{\mathcal{A}}$ if $H$ is triangular in general. The $H$-equivariant $\mathcal{A}$-bimodules are still too arbitrary. One has to demand more symmetry before. We do so by considering a braided commutative left $H$-module algebra $\mathcal{A}$ for a triangular Hopf algebra $(H, \mathcal{R})$ instead of a general left $\mathcal{A}$-module algebra. This means that $b \cdot a=\left(\mathcal{R}_{1}^{-1} \triangleright a\right) \cdot\left(\mathcal{R}_{2}^{-1} \triangleright b\right)$ holds for all $a, b \in \mathcal{A}$. On the level of $\mathcal{A}$-bimodules we want to keep this symmetry: an $H$-equivariant braided symmetric $\mathcal{A}$-bimodule $\mathcal{M}$ for a braided commutative left $H$-module algebra $\mathcal{A}$ is an $H$-equivariant $\mathcal{A}$-bimodule such that $m \cdot a=\left(\mathcal{R}_{1}^{-1} \triangleright a\right) \cdot\left(\mathcal{R}_{2}^{-1} \triangleright m\right)$ for all $a \in \mathcal{A}$ and $m \in \mathcal{M}$. In other words, the left and right $\mathcal{A}$-actions are related via the universal $\mathcal{R}$-matrix, mirroring the braided commutativity of $\mathcal{A}$. These bimodules form a category ${ }_{\mathcal{A}}^{H} \mathcal{M}_{\mathcal{A}}^{\mathcal{R}}$ with morphisms being the usual left $H$-linear and left and right $\mathcal{A}$-linear maps. A proof of the following statement can be found in $[6$ ] Theorem 5.21.

Theorem 2.11. If $H$ is triangular and $\mathcal{A}$ is braided commutative we obtain a braided monoidal equivalence

$$
\left({ }_{\mathcal{A}}^{H} \mathcal{M}_{\mathcal{A}}^{\mathcal{R}}, \otimes_{\mathcal{A}}, c^{\mathcal{R}}\right) \cong\left({ }_{\mathcal{A}_{\mathcal{F}}}^{H_{\mathcal{F}}} \mathcal{M}_{\mathcal{A}_{\mathcal{F}}}^{\mathcal{R}_{\mathcal{F}}}, \otimes_{\mathcal{A}_{\mathcal{F}}}, c^{\mathcal{R}_{\mathcal{F}}}\right)
$$

between braided monoidal categories.

\section{Braided Commutative Geometry}

We enter the main section of these notes with the aim to construct a noncommutative Cartan calculus for any braided commutative algebra. Since its development is entirely parallel to the classical Cartan calculus on a commutative algebra, with basically no choices on the way, it feels justified to call it the braided Cartan calculus on a fixed braided commutative algebra. Before proving this result we recall the notion of multivector fields and differential forms on a commutative algebra, also to indicate the naturality of the generalization. The corresponding Graßmann and Gerstenhaber structures are equivariant with respect to a cocommutative Hopf algebra if the commutative algebra is a Hopf algebra module algebra in addition. More in general we give the definitions of braided Graßmann and Gerstenhaber algebra and provide braided multivector fields and differential forms on a braided commutative algebra as examples. In the second subsection we introduce a differential on braided differential forms via a braided version of the Chevalley-Eilenberg formula. Remark that the differential is a graded braided derivation with respect to the braided wedge product, however, since it is equivariant, it resembles a graded (non-braided) derivation. Using graded braided commutators the relations between the braided Lie derivative, insertion and differential are generalizing and entirely mirror the commutation relations of the classical Cartan calculus. We end the second subsection by applying the gauge equivalence given by the Drinfel'd functor to the braided Cartan calculus and proving that the result is isomorphic to the braided Cartan calculus on the twisted algebra with respect to the twisted triangular structure. Some ramifications of 
this gauge equivalence, in particular for the interpretation of the twisted Cartan calculus on a commutative algebra, are discussed. As an application of the braided Cartan calculus the third and last subsection deals with equivariant covariant derivatives and metrics. The main results are the extension of an equivariant covariant derivative to braided multivector fields and differential forms and the existence of a unique equivariant Levi-Civita covariant derivative for a fixed non-degenerate equivariant metric. We prove that the Drinfel'd functor is compatible with all constructions.

\subsection{Braided Graßmann and Gerstenhaber Algebras}

For the Cartan calculus on a commutative algebra $\mathcal{A}$ the two most important $\mathcal{A}$-bimodules are the multivector fields $\mathfrak{X}^{\bullet}(\mathcal{A})$ and differential forms $\Omega^{\bullet}(\mathcal{A})$. They are graded and possess a Graßmann structure. If $\mathcal{A}$ is a left $H$-module algebra for a cocommutative Hopf algebra $H$, $\mathfrak{X}^{\bullet}(\mathcal{A})$ and $\Omega^{\bullet}(\mathcal{A})$ are $H$-equivariant symmetric $\mathcal{A}$-bimodules and the module actions respect the grading. Let us briefly recall the construction of those modules and then generalize them to the category ${ }_{\mathcal{A}}^{H} \mathcal{M}_{\mathcal{A}}^{\mathcal{R}}$ for a triangular Hopf algebra $(H, \mathcal{R})$ and a braided commutative left $H$-module algebra $\mathcal{A}$.

Fix a cocommutative Hopf algebra $H$ and a commutative left $H$-module algebra $\mathcal{A}$ for the moment. The derivations $\operatorname{Der}(\mathcal{A})$ of $\mathcal{A}$ are an $H$-equivariant symmetric $\mathcal{A}$-bimodule with left $H$-action given by the adjoint action

$$
(\xi \triangleright X)(a)=\xi_{(1)} \triangleright\left(X\left(S\left(\xi_{(2)}\right) \triangleright a\right)\right)
$$

and left and right $\mathcal{A}$-module actions $(a \cdot X)(b)=a \cdot X(b)=(X \cdot a)(b)$, for all $\xi \in H, X \in \operatorname{Der}(\mathcal{A})$ and $a \in \mathcal{A}$. In particular, the tensor algebra

$$
\mathrm{T}^{\bullet} \operatorname{Der}(\mathcal{A})=\mathcal{A} \oplus \operatorname{Der}(\mathcal{A}) \oplus\left(\operatorname{Der}(\mathcal{A}) \otimes_{\mathcal{A}} \operatorname{Der}(\mathcal{A})\right) \oplus \cdots
$$

of $\operatorname{Der}(\mathcal{A})$ with respect to the tensor product $\otimes_{\mathcal{A}}$ over $\mathcal{A}$ is well-defined. It is an $H$-equivariant symmetric $\mathcal{A}$-bimodule with module actions defined on factorizing elements $X_{1} \otimes_{\mathcal{A}} \cdots \otimes_{\mathcal{A}} X_{k} \in$ $\mathrm{T}^{k} \operatorname{Der}(\mathcal{A})$ by

$$
\begin{aligned}
\xi \triangleright\left(X_{1} \otimes_{\mathcal{A}} \cdots \otimes_{\mathcal{A}} X_{k}\right) & =\left(\xi_{(1)} \triangleright X_{1}\right) \otimes_{\mathcal{A}} \cdots \otimes_{\mathcal{A}}\left(\xi_{(k)} \triangleright X_{k}\right), \\
a \cdot\left(X_{1} \otimes_{\mathcal{A}} \cdots \otimes_{\mathcal{A}} X_{k}\right) & =\left(a \cdot X_{1}\right) \otimes_{\mathcal{A}} \cdots \otimes_{\mathcal{A}} X_{k}, \\
\left(X_{1} \otimes_{\mathcal{A}} \cdots \otimes_{\mathcal{A}} X_{k}\right) \cdot a & =X_{1} \otimes_{\mathcal{A}} \cdots \otimes_{\mathcal{A}}\left(X_{k} \cdot a\right)
\end{aligned}
$$

for all $\xi \in H$ and $a \in \mathcal{A}$. Furthermore, there is an ideal $I$ in $\mathrm{T}^{\bullet} \operatorname{Der}(\mathcal{A})$ generated by elements $X_{1} \otimes_{\mathcal{A}} \cdots \otimes_{\mathcal{A}} X_{k} \in \mathrm{T}^{k} \operatorname{Der}(\mathcal{A})$ such that $X_{i}=X_{j}$ for a pair $(i, j)$ such that $1 \leq i<j \leq k$. The quotient $\mathrm{T}^{\bullet} \operatorname{Der}(\mathcal{A}) / I$ is the exterior algebra. It is the Graßmann algebra $\mathfrak{X}^{\bullet}(\mathcal{A})$ of multivector fields on $\mathcal{A}$ and the induced product, the wedge product, is denoted by $\wedge$. Since $H$ is cocommutative and the $\mathcal{A}$-actions symmetric, they respect the ideal $I$. Consequently, the induced actions on $\mathfrak{X}^{\bullet}(\mathcal{A})$ are well-defined, structuring the multivector fields as an $H$-equivariant symmetric $\mathcal{A}$-bimodule with the additional property that the module actions respect the grading. Moreover, the usual commutator of endomorphisms $[\cdot, \cdot]$ is a Lie bracket for the derivations of $\mathcal{A}$. It extends uniquely to a Gerstenhaber bracket $\llbracket \cdot, \cdot \rrbracket$ on $\mathfrak{X}^{\bullet}(\mathcal{A})$ by defining $\llbracket a, b \rrbracket=0$, $\llbracket X, a \rrbracket=X(a)$ for all $a, b \in \mathcal{A}, X \in \operatorname{Der}(\mathcal{A})$ and inductively declaring the graded Leibniz rule

$$
\llbracket X, Y \wedge Z \rrbracket=\llbracket X, Y \rrbracket \wedge Z+(-1)^{(k-1) \ell} Y \wedge \llbracket X, Z \rrbracket
$$

for all $X \in \mathfrak{X}^{k}(\mathcal{A}), Y \in \mathfrak{X}^{\ell}(\mathcal{A})$ and $Z \in \mathfrak{X}^{\bullet}(\mathcal{A})$. In detail this means that $\llbracket \cdot, \rrbracket: \mathfrak{X}^{k}(\mathcal{A}) \times$ $\mathfrak{X}^{\ell}(\mathcal{A}) \rightarrow \mathfrak{X}^{k+\ell-1}(\mathcal{A})$ is a graded (with respect to the degree shifted by -1 ) Lie bracket, i.e. it is graded skew-symmetric

$$
\llbracket Y, X \rrbracket=-(-1)^{(k-1)(\ell-1)} \llbracket X, Y \rrbracket
$$

and satisfies the graded Jacobi identity

$$
\llbracket X, \llbracket Y, Z \rrbracket \rrbracket=\llbracket \llbracket X, Y \rrbracket, Z \rrbracket+(-1)^{(k-1)(\ell-1)} \llbracket Y, \llbracket X, Z \rrbracket \rrbracket,
$$

where $X \in \mathfrak{X}^{k}(\mathcal{A}), Y \in \mathfrak{X}^{\ell}(\mathcal{A})$ and $Z \in \mathfrak{X}^{\bullet}(\mathcal{A})$, such that the graded Leibniz rule (3.3) holds in addition. Using the formula

$$
\begin{aligned}
\llbracket X_{1} \wedge \cdots \wedge X_{k}, Y_{1} \wedge \cdots \wedge Y_{\ell} \rrbracket= & \sum_{i=1}^{k} \sum_{j=1}^{\ell}(-1)^{i+j}\left[X_{i}, Y_{j}\right] \wedge X_{1} \wedge \cdots \wedge \widehat{X_{i}} \wedge \cdots \wedge X_{k} \\
& \wedge Y_{1} \wedge \cdots \wedge \widehat{Y_{j}} \wedge \cdots \wedge Y_{\ell},
\end{aligned}
$$


which holds for all $X_{1}, \ldots, X_{k}, Y_{1}, \ldots, Y_{\ell} \in \mathfrak{X}^{1}(\mathcal{A})$, it is easy to prove that the Gerstenhaber bracket $\llbracket \cdot, \cdot \rrbracket$ is $H$-equivariant, i.e. that

$$
\xi \triangleright \llbracket X, Y \rrbracket=\llbracket \xi_{(1)} \triangleright X, \xi_{(2)} \triangleright Y \rrbracket
$$

for all $\xi \in H$ and $X, Y \in \mathfrak{X}^{\bullet}(\mathcal{A})$. Note that $\widehat{X}_{i}$ and $\widehat{Y_{j}}$ means that $X_{i}$ and $Y_{j}$ are left out in the wedge product of eq.(3.6).

Differential forms on $\mathcal{A}$ are defined in the following way: consider $\operatorname{Hom}_{\mathcal{A}}(\operatorname{Der}(\mathcal{A}), \mathcal{A})$, the $\mathbb{k}$-module of $\mathbb{k}$-linear and $\mathcal{A}$-linear maps $\operatorname{Der}(\mathcal{A}) \rightarrow \mathcal{A}$. It is an $H$-equivariant symmetric $\mathcal{A}$ bimodule with respect to the adjoint $H$-action and $(a \cdot \omega)(X)=a \cdot \omega(X)=(\omega \cdot a)(X)$ for all $a \in \mathcal{A}, \omega \in \operatorname{Hom}_{\mathcal{A}}(\operatorname{Der}(\mathcal{A}), \mathcal{A})$ and $X \in \operatorname{Der}(\mathcal{A})$. The corresponding exterior algebra is denoted by $\underline{\Omega}^{\bullet}(\mathcal{A})$. One can define a differential d of $\omega \in \underline{\Omega}^{k}(\mathcal{A})$ via

$$
\begin{gathered}
(\mathrm{d} \omega)\left(X_{1}, \ldots, X_{k+1}\right)=\sum_{i=1}^{k+1}(-1)^{i+1} X_{i}\left(\omega\left(X_{1}, \ldots, \widehat{X_{i}}, \ldots, X_{k+1}\right)\right) \\
+\sum_{i<j}(-1)^{i+j} \omega\left(\left[X_{i}, X_{j}\right], X_{1}, \ldots, \widehat{X_{i}}, \ldots, \widehat{X_{j}}, \ldots, X_{k+1}\right)
\end{gathered}
$$

for all $X_{1}, \ldots, X_{k+1} \in \operatorname{Der}(\mathcal{A})$. This is known as the Chevalley-Eilenberg formula. Define now the differential forms $\Omega^{\bullet}(\mathcal{A})$ on $\mathcal{A}$ to be the smallest differential graded subalgebra of $\underline{\Omega}^{\bullet}(\mathcal{A})$ such that $\mathcal{A} \subseteq \Omega^{\bullet}(\mathcal{A})$ (compare to [18, 19]). In this case every element of $\Omega^{k}(\mathcal{A})$ can be written as a finite sum of elements of the form $a_{0} \mathrm{~d} a_{1} \wedge \ldots \wedge \mathrm{d} a_{k}$, where $a_{0}, \ldots, a_{k} \in \mathcal{A}$. The induced actions structure $\left(\Omega^{\bullet}(\mathcal{A}), \wedge\right)$ as an $H$-equivariant symmetric $\mathcal{A}$-bimodule and a Graßmann algebra such that $\wedge$ is equivariant and $H \triangleright \Omega^{k}(\mathcal{A}) \subseteq \Omega^{k}(\mathcal{A})$. From the Chevalley-Eilenberg formula it follows that $\mathrm{d}$ commutes with $\triangleright$. The insertion $\mathrm{i}: \mathfrak{X}^{1}(\mathcal{A}) \otimes \Omega^{k}(\mathcal{A}) \rightarrow \Omega^{k-1}(\mathcal{A})$ of derivations $X \in \mathfrak{X}^{1}(\mathcal{A})$ into the first slot of a differential form, i.e. $\left(\mathrm{i}_{X}(\omega)\right)\left(X_{1}, \ldots, X_{k-1}\right)=$ $\omega\left(X, X_{1}, \ldots, X_{k-1}\right)$ for all $\omega \in \Omega^{k}(\mathcal{A})$ and $X_{1}, \ldots, X_{k-1} \in \mathfrak{X}^{1}(\mathcal{A})$ is $H$-equivariant.

We are ready to generalize the concepts of Graßmann and Gerstenhaber algebra to the setting of equivariant braided symmetric bimodules. This is exemplified by the example of braided multivector fields. Fix a triangular Hopf algebra $(H, \mathcal{R})$ and a braided commutative left $H$-module algebra $\mathcal{A}$. A $\mathbb{k}$-linear endomorphism $X$ of $\mathcal{A}$ is said to be a braided derivation if

$$
X(a b)=X(a) b+\left(\mathcal{R}_{1}^{-1} \triangleright a\right)\left(\left(\mathcal{R}_{2}^{-1} \triangleright X\right)(b)\right)
$$

for all $a, b \in \mathcal{A}$, where the left $H$-action on endomorphisms is given by the adjoint action.

Lemma 3.1. The braided derivations $\operatorname{Der}_{\mathcal{R}}(\mathcal{A})$ are an $H$-equivariant braided symmetric $\mathcal{A}$ bimodule. Furthermore, the braided commutator

$$
[X, Y]_{\mathcal{R}}=X Y-\left(\mathcal{R}_{1}^{-1} \triangleright Y\right)\left(\mathcal{R}_{2}^{-1} \triangleright X\right),
$$

where $X, Y \in \operatorname{Der}_{\mathcal{R}}(\mathcal{A})$, structures $\operatorname{Der}_{\mathcal{R}}(\mathcal{A})$ as a braided Lie algebra. The latter means that $[\cdot, \cdot]_{\mathcal{R}}$ is braided skew-symmetric, i.e. $[Y, X]_{\mathcal{R}}=-\left[\mathcal{R}_{1}^{-1} \triangleright X, \mathcal{R}_{2}^{-1} \triangleright Y\right]_{\mathcal{R}}$ and satisfies the braided Jacobi identity, i.e.

$$
\left[X,[Y, Z]_{\mathcal{R}}\right]_{\mathcal{R}}=\left[[X, Y]_{\mathcal{R}}, Z\right]_{\mathcal{R}}+\left[\mathcal{R}_{1}^{-1} \triangleright Y,\left[\mathcal{R}_{2}^{-1} \triangleright X, Z\right]_{\mathcal{R}}\right]_{\mathcal{R}}
$$

for all $X, Y, Z \in \operatorname{Der}_{\mathcal{R}}(\mathcal{A})$.

This is an elementary consequence of the properties of the triangular structure. In a next step we want to generalize the construction of multivector fields of a commutative algebra (compare also to [10]). Since $\operatorname{Der}_{\mathcal{R}}(\mathcal{A})$ is an $\mathcal{A}$-bimodule we can build the tensor algebra $\mathrm{T}^{\bullet} \operatorname{Der}_{\mathcal{R}}(\mathcal{A})$ with respect to $\otimes_{\mathcal{A}}$ and with module actions on factorizing elements $X_{1} \otimes_{\mathcal{A}}$ $\cdots \otimes_{\mathcal{A}} X_{k} \in \mathrm{T}^{k} \operatorname{Der}_{\mathcal{R}}(\mathcal{A})$ defined by

$$
\begin{aligned}
\xi \triangleright\left(X_{1} \otimes_{\mathcal{A}} \cdots \otimes_{\mathcal{A}} X_{k}\right) & =\left(\xi_{(1)} \triangleright X_{1}\right) \otimes_{\mathcal{A}} \cdots \otimes_{\mathcal{A}}\left(\xi_{(k)} \triangleright X_{k}\right), \\
a \cdot\left(X_{1} \otimes_{\mathcal{A}} \cdots \otimes_{\mathcal{A}} X_{k}\right) & =\left(a \cdot X_{1}\right) \otimes_{\mathcal{A}} \cdots \otimes_{\mathcal{A}} X_{k}, \\
\left(X_{1} \otimes_{\mathcal{A}} \cdots \otimes_{\mathcal{A}} X_{k}\right) \cdot a & =X_{1} \otimes_{\mathcal{A}} \cdots \otimes_{\mathcal{A}}\left(X_{k} \cdot a\right)
\end{aligned}
$$

for all $\xi \in H$ and $a \in \mathcal{A}$. There is an ideal $I$ in $\mathrm{T}^{\bullet} \operatorname{Der}_{\mathcal{R}}(\mathcal{A})$ generated by elements $X_{1} \otimes_{\mathcal{A}}$ $\cdots \otimes_{\mathcal{A}} X_{k} \in \mathrm{T}^{k} \operatorname{Der}_{\mathcal{R}}(\mathcal{A})$ which equal

$$
\begin{gathered}
X_{1} \otimes_{\mathcal{A}} \cdots \otimes_{\mathcal{A}} X_{i-1} \otimes_{\mathcal{A}}\left(\mathcal{R}_{1}^{\prime-1} \triangleright\left(\left(\mathcal{R}_{1}^{-1} \triangleright X_{j}\right) \otimes_{\mathcal{A}}\left(\mathcal{R}_{2}^{-1} \triangleright\left(X_{i+1} \otimes_{\mathcal{A}} \cdots \otimes_{\mathcal{A}} X_{j-1}\right)\right)\right)\right) \\
\otimes_{\mathcal{A}}\left(\mathcal{R}_{2}^{\prime-1} \triangleright X_{i}\right) \otimes_{\mathcal{A}} X_{j+1} \otimes_{\mathcal{A}} \cdots \otimes_{\mathcal{A}} X_{k}
\end{gathered}
$$


for a pair $(i, j)$ such that $1 \leq i<j \leq k$. One can prove that the module actions (3.12) respect $I$ (see [4]). This induces an $H$-equivariant graded associative braided commutative product $\wedge_{\mathcal{R}}$ on the quotient, declaring the braided multivector fields $\left(\mathfrak{X}_{\mathcal{R}}^{\bullet}(\mathcal{A}), \wedge_{\mathcal{R}}\right)$ on $\mathcal{A}$. In general, the associative unital graded algebra and $H$-equivariant braided symmetric $\mathcal{A}$-bimodule $\left(\Lambda^{\bullet} \mathcal{M}, \wedge_{\mathcal{R}}\right)$ associated to an $H$-equivariant braided symmetric $\mathcal{A}$-bimodule $\mathcal{M}$ in this way is said to be the braided Graßmann algebra or braided exterior algebra corresponding to $\mathcal{M}$. Coming back to the example of braided multivector fields we can use the braided commutator of vector fields to obtain additional structure on the braided Graßmann algebra. Namely, we are defining a $\mathbb{k}$-bilinear operation $\llbracket \cdot, \cdot \rrbracket_{\mathcal{R}}: \mathfrak{X}_{\mathcal{R}}^{k}(\mathcal{A}) \times \mathfrak{X}_{\mathcal{R}}^{\ell}(\mathcal{A}) \rightarrow \mathfrak{X}_{\mathcal{R}}^{k+\ell-1}(\mathcal{A})$ in the following way. If $a, b \in \mathcal{A}$ we set $\llbracket a, b \rrbracket_{\mathcal{R}}=0$. For $a \in \mathcal{A}$ and a factorizing element $X=X_{1} \wedge_{\mathcal{R}} \cdots \wedge_{\mathcal{R}} X_{k} \in \mathfrak{X}_{\mathcal{R}}^{k}(\mathcal{A})$ where $k>0$ we define

$$
\begin{aligned}
\llbracket X, a \rrbracket_{\mathcal{R}}= & \sum_{i=1}^{k}(-1)^{k-i} X_{1} \wedge_{\mathcal{R}} \cdots \wedge_{\mathcal{R}} X_{i-1} \wedge_{\mathcal{R}}\left(X_{i}\left(\mathcal{R}_{1}^{-1} \triangleright a\right)\right) \\
& \wedge_{\mathcal{R}}\left(\mathcal{R}_{2}^{-1} \triangleright\left(X_{i+1} \wedge_{\mathcal{R}} \cdots \wedge_{\mathcal{R}} X_{k}\right)\right)
\end{aligned}
$$

and

$$
\begin{aligned}
\llbracket a, X \rrbracket_{\mathcal{R}}= & \sum_{i=1}^{k}(-1)^{i}\left(\mathcal{R}_{1(1)}^{-1} \triangleright\left(X_{1} \wedge_{\mathcal{R}} \cdots \wedge_{\mathcal{R}} X_{i-1}\right)\right) \wedge_{\mathcal{R}}\left(\left(\mathcal{R}_{1(2)}^{-1} \triangleright X_{i}\right)\left(\mathcal{R}_{2}^{-1} \triangleright a\right)\right) \\
& \wedge_{\mathcal{R}} X_{i+1} \wedge_{\mathcal{R}} \cdots \wedge_{\mathcal{R}} X_{k} .
\end{aligned}
$$

Furthermore, on factorizing elements $X=X_{1} \wedge_{\mathcal{R}} \cdots \wedge_{\mathcal{R}} X_{k} \in \mathfrak{X}_{\mathcal{R}}^{k}(\mathcal{A})$ and $Y=Y_{1} \wedge_{\mathcal{R}} \cdots \wedge_{\mathcal{R}} Y_{\ell} \in$ $\mathfrak{X}_{\mathcal{R}}^{\ell}(\mathcal{A})$, where $k, \ell>0$, we define

$$
\begin{aligned}
& \llbracket X, Y \rrbracket_{\mathcal{R}}=\sum_{i=1}^{k} \sum_{j=1}^{\ell}(-1)^{i+j}\left[\mathcal{R}_{1}^{-1} \triangleright X_{i}, \mathcal{R}_{1}^{\prime-1} \triangleright Y_{j}\right]_{\mathcal{R}} \\
& \wedge_{\mathcal{R}}\left(\mathcal { R } _ { 2 } ^ { \prime - 1 } \triangleright \left(\left(\mathcal{R}_{2}^{-1} \triangleright\left(X_{1} \wedge_{\mathcal{R}} \cdots \wedge_{\mathcal{R}} X_{i-1}\right)\right) \wedge_{\mathcal{R}} \widehat{X}_{i} \wedge_{\mathcal{R}} X_{i+1} \wedge_{\mathcal{R}} \cdots \wedge_{\mathcal{R}} X_{k}\right.\right. \\
& \left.\left.\wedge_{\mathcal{R}} Y_{1} \wedge_{\mathcal{R}} \cdots \wedge_{\mathcal{R}} Y_{j-1}\right)\right) \wedge_{\mathcal{R}} \widehat{Y}_{j} \wedge_{\mathcal{R}} Y_{j+1} \wedge_{\mathcal{R}} \cdots \wedge_{\mathcal{R}} Y_{\ell}
\end{aligned}
$$

where $[\cdot, \cdot]_{\mathcal{R}}$ denotes the braided commutator and $\widehat{X}_{i}$ and $\widehat{Y_{j}}$ means that $X_{i}$ and $Y_{j}$ are omitted in above product. The operation $\llbracket \cdot, \cdot \rrbracket_{\mathcal{R}}$ is said to be the braided Schouten-Nijenhuis bracket.

Proposition 3.2. The braided multivector fields $\left(\mathfrak{X}_{\mathcal{R}}^{\bullet}(\mathcal{A}), \wedge_{\mathcal{R}}, \llbracket \cdot, \cdot \rrbracket_{\mathcal{R}}\right)$ on $\mathcal{A}$ are an associative unital graded algebra and an $H$-equivariant braided symmetric $\mathcal{A}$-bimodule equipped with an $H$ equivariant graded (with degree shifted by -1 ) braided Lie bracket $\llbracket \cdot, \cdot \rrbracket_{\mathcal{R}}: \mathfrak{X}_{\mathcal{R}}^{k}(\mathcal{A}) \otimes \mathfrak{X}_{\mathcal{R}}^{\ell}(\mathcal{A}) \rightarrow$ $\mathfrak{X}_{\mathcal{R}}^{k+\ell-1}(\mathcal{A})$, which means that $\llbracket \cdot, \cdot \rrbracket_{\mathcal{R}}$ is graded braided skewsymmetric, i.e.

$$
\llbracket Y, X \rrbracket_{\mathcal{R}}=-(-1)^{(k-1) \cdot(\ell-1)} \llbracket \mathcal{R}_{1}^{-1} \triangleright X, \mathcal{R}_{2}^{-1} \triangleright Y \rrbracket_{\mathcal{R}}
$$

and satisfies the graded braided Jacobi identity

$$
\llbracket X, \llbracket Y, Z \rrbracket_{\mathcal{R}} \rrbracket_{\mathcal{R}}=\llbracket \llbracket X, Y \rrbracket_{\mathcal{R}}, Z \rrbracket_{\mathcal{R}}+(-1)^{(k-1) \cdot(\ell-1)} \llbracket \mathcal{R}_{1}^{-1} \triangleright Y, \llbracket \mathcal{R}_{2}^{-1} \triangleright X, Z \rrbracket_{\mathcal{R}} \rrbracket_{\mathcal{R}},
$$

such that the graded braided Leibniz rule

$$
\llbracket X, Y \wedge_{\mathcal{R}} Z \rrbracket_{\mathcal{R}}=\llbracket X, Y \rrbracket_{\mathcal{R}} \wedge_{\mathcal{R}} Z+(-1)^{(k-1) \cdot \ell}\left(\mathcal{R}_{1}^{-1} \triangleright Y\right) \wedge_{\mathcal{R}} \llbracket \mathcal{R}_{2}^{-1} \triangleright X, Z \rrbracket_{\mathcal{R}}
$$

holds in addition, where $X \in \mathfrak{X}_{\mathcal{R}}^{k}(\mathcal{A}), Y \in \mathfrak{X}_{\mathcal{R}}^{\ell}(\mathcal{A})$ and $Z \in \mathfrak{X}_{\mathcal{R}}^{\bullet}(\mathcal{A})$.

More in general we make the following definition.

Definition 3.3 (Braided Gerstenhaber algebra). An associative unital graded algebra and $H$-equivariant braided symmetric $\mathcal{A}$-bimodule $\left(\mathfrak{G}^{\bullet}, \wedge_{\mathcal{R}}\right)$ is said to be a braided Gerstenhaber algebra if the module actions respect the degree and if there is an $H$-equivariant graded (with degree shifted by -1$)$ braided Lie bracket satisfying a graded braided Leibniz rule with respect to $\wedge_{\mathcal{R}}$.

Let $\mathfrak{G}^{\bullet}$ be a braided Gerstenhaber algebra. It follows that $\mathfrak{G}^{0}$ is a braided commutative left $H$-module algebra and $\mathfrak{G}^{1}$ is a braided Lie algebra. Moreover, $\mathfrak{G}^{1}$ is an $H$-equivariant braided symmetric $\mathfrak{G}^{0}$-bimodule and $\mathfrak{G}^{k}$ is an $H$-equivariant braided symmetric $\mathfrak{G}^{1}$-bimodule. This means that for any $X \in \mathfrak{G}^{1}$ we can define the braided Lie derivative $\mathscr{L}_{X}^{\mathcal{R}}=\llbracket X, \rrbracket_{\mathcal{R}}: \mathfrak{G}^{k} \rightarrow \mathfrak{G}^{k}$ which is a braided derivation, i.e.

$$
\mathscr{L}_{X}^{\mathcal{R}}\left(Y \wedge_{\mathcal{R}} Z\right)=\mathscr{L}_{X}^{\mathcal{R}} Y \wedge_{\mathcal{R}} Z+\left(\mathcal{R}_{1}^{-1} \triangleright Y\right) \wedge_{\mathcal{R}}\left(\mathcal{R}_{2}^{-1} \triangleright \mathscr{L}_{X}^{\mathcal{R}}\right) Z
$$


for all $X \in \mathfrak{G}^{1}$ and $Y, Z \in \mathfrak{G}^{\bullet}$. It furthermore satisfies $\mathscr{L}_{[X, Y]_{\mathcal{R}}}^{\mathcal{R}}=\mathscr{L}_{X}^{\mathcal{R}} \mathscr{L}_{Y}^{\mathcal{R}}-\mathscr{L}_{\mathcal{R}_{1}^{-1} \triangleright Y^{\mathcal{R}}}^{\mathscr{L}_{\mathcal{R}_{2}^{-1}}^{\mathcal{R}} \triangleright X}$ for all $X, Y \in \mathfrak{G}^{1}$. On the other hand one can start with a braided commutative left $H$-module algebra $\mathcal{A}$ and construct the braided Gerstenhaber algebra of its braided multivector fields, as discussed before. Note that the braided Schouten-Nijenhuis bracket $\llbracket \cdot, \cdot \rrbracket_{\mathcal{R}}$ is the unique braided Gerstenhaber bracket on $\left(\mathfrak{X}_{\mathcal{R}}^{\bullet}(\mathcal{A}), \wedge_{\mathcal{R}}\right)$ such that

$$
\llbracket X, a \rrbracket_{\mathcal{R}}=X(a) \text { and } \llbracket X, Y \rrbracket_{\mathcal{R}}=[X, Y]_{\mathcal{R}}
$$

hold for all $a \in \mathcal{A}$ and $X, Y \in \mathfrak{X}_{\mathcal{R}}^{1}(\mathcal{A})$.

Dually we consider $\mathbb{k}$-linear maps $\omega: \operatorname{Der}_{\mathcal{R}}(\mathcal{A}) \rightarrow \mathcal{A}$ such that $\omega(X \cdot a)=\omega(X) \cdot a$ for all $X \in \operatorname{Der}_{\mathcal{R}}(\mathcal{A})$ and $a \in \mathcal{A}$ and denote the corresponding $\mathbb{k}$-module by $\underline{\Omega}_{\mathcal{R}}^{1}(\mathcal{A})$. We structure $\underline{\Omega}_{\mathcal{R}}^{1}(\mathcal{A})$ as a braided symmetric $\mathcal{A}$-bimodule with left and right $\mathcal{A}$-actions defined by

$$
(a \cdot \omega)(X)=a \cdot \omega(X) \text { and }(\omega \cdot a)(X)=\omega\left(\mathcal{R}_{1}^{-1} \triangleright X\right) \cdot\left(\mathcal{R}_{2}^{-1} \triangleright a\right),
$$

respectively, and left $H$-action $(\xi \triangleright \omega)(X)=\xi_{(1)} \triangleright\left(\omega\left(S\left(\xi_{(2)}\right) \triangleright X\right)\right)$, the adjoint action, for all $\xi \in H, a \in \mathcal{A}, \omega \in \underline{\Omega}_{\mathcal{R}}^{1}(\mathcal{A})$ and $X \in \operatorname{Der}_{\mathcal{R}}(\mathcal{A})$. It follows that $\omega(a \cdot X)=\left(\mathcal{R}_{1}^{-1} \triangleright a\right) \cdot\left(\mathcal{R}_{2}^{-1} \triangleright \omega\right)(X)$ and $\xi \triangleright(\omega(X))=\left(\xi_{(1)} \triangleright \omega\right)\left(\xi_{(2)} \triangleright X\right)$ for all $\xi \in H, \omega \in \underline{\Omega}_{\mathcal{R}}^{1}(\mathcal{A}), a \in \mathcal{A}$ and $X \in \operatorname{Der}_{\mathcal{R}}(\mathcal{A})$. There is an $H$-equivariant insertion $\mathrm{i}^{\mathcal{R}}: \mathfrak{X}_{\mathcal{R}}^{1}(\mathcal{A}) \otimes \underline{\Omega}_{\mathcal{R}}^{1}(\mathcal{A}) \rightarrow \mathcal{A}$, defined for any $X \in \operatorname{Der}_{\mathcal{R}}(\mathcal{A})$ and $\omega \in \underline{\Omega}_{\mathcal{R}}^{1}(\mathcal{A})$ by i $i_{X}^{\mathcal{R}} \omega=\left(\mathcal{R}_{1}^{-1} \triangleright \omega\right)\left(\mathcal{R}_{2}^{-1} \triangleright X\right)$. In fact,

$$
\begin{aligned}
\xi \triangleright\left(\mathrm{i}_{X}^{\mathcal{R}} \omega\right) & =\xi \triangleright\left(\left(\mathcal{R}_{1}^{-1} \triangleright \omega\right)\left(\mathcal{R}_{2}^{-1} \triangleright X\right)\right)=\left(\left(\xi_{(1)} \mathcal{R}_{1}^{-1}\right) \triangleright \omega\right)\left(\left(\xi_{(2)} \mathcal{R}_{2}^{-1}\right) \triangleright X\right) \\
& =\left(\left(\mathcal{R}_{1}^{-1} \xi_{(2)}\right) \triangleright \omega\right)\left(\left(\mathcal{R}_{2}^{-1} \xi_{(1)}\right) \triangleright X\right)=\mathrm{i}_{\xi_{(1)}}^{\mathcal{R}} \triangleright X\left(\xi_{(2)} \triangleright \omega\right)
\end{aligned}
$$

for all $\xi \in H, X \in \operatorname{Der}_{\mathcal{R}}(\mathcal{A})$ and $\omega \in \underline{\Omega}_{\mathcal{R}}^{1}(\mathcal{A})$. It follows that the braided exterior algebra $\underline{\Omega}_{\mathcal{R}}^{\bullet}(\mathcal{A})$ of $\underline{\Omega}_{\mathcal{R}}^{1}(\mathcal{A})$ is an $H$-equivariant braided symmetric $\mathcal{A}$-bimodule. In the following lines we show that it is also compatible with the braided evaluation. For $\omega, \eta \in \underline{\Omega}_{\mathcal{R}}^{1}(\mathcal{A})$ we define a $\mathbb{k}$-bilinear map $\omega \wedge_{\mathcal{R}} \eta: \operatorname{Der}(\mathcal{A}) \times \operatorname{Der}(\mathcal{A}) \rightarrow \mathcal{A}$ by

$$
\left(\omega \wedge_{\mathcal{R}} \eta\right)(X, Y)=\left(\omega\left(\mathcal{R}_{1}^{-1} \triangleright X\right)\right)\left(\left(\mathcal{R}_{2}^{-1} \triangleright \eta\right)(Y)\right)-\left(\omega\left(\mathcal{R}_{1}^{-1} \triangleright Y\right)\right)\left(\left(\mathcal{R}_{2(1)}^{-1} \triangleright \eta\right)\left(\mathcal{R}_{2(2)}^{-1} \triangleright X\right)\right)
$$

for all $X, Y \in \operatorname{Der}_{\mathcal{R}}(\mathcal{A})$. One proves that

$$
-\left(\omega \wedge_{\mathcal{R}} \eta\right)\left(\mathcal{R}_{1}^{-1} \triangleright Y, \mathcal{R}_{2}^{-1} \triangleright X\right)=\left(\omega \wedge_{\mathcal{R}} \eta\right)(X, Y)=-\left(\left(\mathcal{R}_{1}^{-1} \triangleright \eta\right) \wedge_{\mathcal{R}}\left(\mathcal{R}_{2}^{-1} \triangleright \omega\right)\right)(X, Y)
$$

and that

$$
\begin{aligned}
\left(\omega \wedge_{\mathcal{R}} \eta\right)(X, Y \cdot a) & =\left(\left(\omega \wedge_{\mathcal{R}} \eta\right)(X, Y)\right) \cdot a, \\
\left(\omega \wedge_{\mathcal{R}} \eta\right)(a \cdot X, Y) & =\left(\mathcal{R}_{1}^{-1} \triangleright a\right) \cdot\left(\left(\mathcal{R}_{2}^{-1} \triangleright\left(\omega \wedge_{\mathcal{R}} \eta\right)\right)(X, Y)\right), \\
\xi \triangleright\left(\left(\omega \wedge_{\mathcal{R}} \eta\right)(X, Y)\right) & =\left(\left(\xi_{(1)} \triangleright \omega\right) \wedge_{\mathcal{R}}\left(\xi_{(2)} \triangleright \eta\right)\right)\left(\xi_{(3)} \triangleright X, \xi_{(4)} \triangleright Y\right)
\end{aligned}
$$

hold for all $\xi \in H, \omega, \eta \in \underline{\Omega}_{\mathcal{R}}^{1}(\mathcal{A}), a \in \mathcal{A}$ and $X, Y \in \operatorname{Der}_{\mathcal{R}}(\mathcal{A})$. The evaluations of the $H$-action and $\mathcal{A}$-module actions read

$$
\begin{aligned}
\left(\xi \triangleright\left(\omega \wedge_{\mathcal{R}} \eta\right)\right)(X, Y) & =\xi_{(1)} \triangleright\left(\left(\omega \wedge_{\mathcal{R}} \eta\right)\left(S\left(\xi_{(3)}\right) \triangleright X, S\left(\xi_{(2)}\right) \triangleright Y\right)\right), \\
\left(a \cdot\left(\omega \wedge_{\mathcal{R}} \eta\right)\right)(X, Y) & =a \cdot\left(\left(\omega \wedge_{\mathcal{R}} \eta\right)(X, Y)\right), \\
\left(\left(\omega \wedge_{\mathcal{R}} \eta\right) \cdot a\right)(X, Y) & =\left(\left(\omega \wedge_{\mathcal{R}} \eta\right)\left(\mathcal{R}_{1(1)}^{-1} \triangleright X, \mathcal{R}_{1(2)}^{-1} \triangleright Y\right)\right) \cdot\left(\mathcal{R}_{2}^{-1} \triangleright a\right) .
\end{aligned}
$$

Inductively one defines the evaluation of higher wedge products. Explicitly, the evaluated module actions on factorizing elements $\omega_{1} \wedge_{\mathcal{R}} \ldots \wedge_{\mathcal{R}} \omega_{k} \in \underline{\Omega}_{\mathcal{R}}^{k}(\mathcal{A}) \mathrm{read}$

$$
\begin{gathered}
\left(\xi \triangleright\left(\omega_{1} \wedge_{\mathcal{R}} \ldots \wedge_{\mathcal{R}} \omega_{k}\right)\right)\left(X_{1}, \ldots, X_{k}\right) \\
=\xi_{(1)} \triangleright\left(\left(\omega_{1} \wedge_{\mathcal{R}} \ldots \wedge_{\mathcal{R}} \omega_{k}\right)\left(S\left(\xi_{(k+1)}\right) \triangleright X_{1}, \ldots, S\left(\xi_{(2)}\right) \triangleright X_{k}\right)\right), \\
\left(a \cdot\left(\omega_{1} \wedge_{\mathcal{R}} \ldots \wedge_{\mathcal{R}} \omega_{k}\right)\right)\left(X_{1}, \ldots, X_{k}\right)=a \cdot\left(\left(\omega_{1} \wedge_{\mathcal{R}} \ldots \wedge_{\mathcal{R}} \omega_{k}\right)\left(X_{1}, \ldots, X_{k}\right)\right)
\end{gathered}
$$

and

$$
\begin{aligned}
& \left(\left(\omega_{1} \wedge_{\mathcal{R}} \ldots \wedge_{\mathcal{R}} \omega_{k}\right) \cdot a\right)\left(X_{1}, \ldots, X_{k}\right) \\
& \quad=\left(\left(\omega_{1} \wedge_{\mathcal{R}} \ldots \wedge_{\mathcal{R}} \omega_{k}\right)\left(\mathcal{R}_{1(1)}^{-1} \triangleright X_{1}, \ldots, \mathcal{R}_{1(k)}^{-1} \triangleright X_{k}\right)\right) \cdot\left(\mathcal{R}_{2}^{-1} \triangleright a\right) .
\end{aligned}
$$

for all $X_{1}, \ldots, X_{k} \in \operatorname{Der}_{\mathcal{R}}(\mathcal{A}), a \in \mathcal{A}$ and $\xi \in H$. It is useful to further define the insertion $\mathrm{i}_{X}^{\mathcal{R}}: \underline{\Omega}_{\mathcal{R}}^{\bullet}(\mathcal{A}) \rightarrow \underline{\Omega}_{\mathcal{R}}^{\bullet-1}(\mathcal{A})$ of an element $X \in \operatorname{Der}_{\mathcal{R}}(\mathcal{A})$ into the last slot an element $\omega \in \underline{\Omega}_{\mathcal{R}}^{k}(\mathcal{A})$ by

Inductively we set

$$
\mathrm{i}_{X}^{\mathcal{R}} \omega=(-1)^{k-1}\left(\mathcal{R}_{1}^{-1} \triangleright \omega\right)\left(\cdot, \ldots, \cdot, \mathcal{R}_{2}^{-1} \triangleright X\right) .
$$

for all $X, Y \in \mathfrak{X}_{\mathcal{R}}^{\bullet}(\mathcal{A})$.

$$
\mathrm{i}_{X \wedge_{\mathcal{R}} Y}^{\mathcal{R}}=\mathrm{i}_{X}^{\mathcal{R}} \mathrm{i}_{Y}^{\mathcal{R}}
$$


Lemma 3.4. $\left(\underline{\Omega}_{\mathcal{R}}^{\bullet}(\mathcal{A}), \wedge_{\mathcal{R}}\right)$ is a graded braided commutative associative unital algebra and an $H$-equivariant braided symmetric $\mathcal{A}$-bimodule. The insertion

$$
\mathrm{i}^{\mathcal{R}}: \mathfrak{X}_{\mathcal{R}}^{\bullet}(\mathcal{A}) \otimes \underline{\Omega}_{\mathcal{R}}^{\bullet}(\mathcal{A}) \rightarrow \underline{\Omega}_{\mathcal{R}}^{\bullet}(\mathcal{A})
$$

of braided multivector fields is $H$-equivariant such that $\mathrm{i}_{X}^{\mathcal{R}}$ is a right $\mathcal{A}$-linear and braided left $\mathcal{A}$-linear homogeneous map of degree $-k$ for all $X \in \mathfrak{X}_{\mathcal{R}}^{k}(\mathcal{A})$. Furthermore, $\mathrm{i}_{X}^{\mathcal{R}}$ is left $\mathcal{A}$-linear and braided right $\mathcal{A}$-linear in $X$. If $k=1 \mathrm{i}_{X}^{\mathcal{R}}$ is a graded braided derivation of degree -1 .

Proof. Fix $a, b \in \mathcal{A}, X \in \operatorname{Der}_{\mathcal{R}}(\mathcal{A}), \xi \in H$ and $\omega \in \underline{\Omega}_{\mathcal{R}}^{1}(\mathcal{A})$. First of all, the left and right $\mathcal{A}$ and left $H$-module actions are well-defined on $\underline{\Omega}_{\mathcal{R}}^{1}(\overline{\mathcal{A}})$, since $(b \cdot \omega)(X \cdot a)=b \cdot(\omega(X \cdot a))=$ $((b \cdot \omega)(X)) \cdot a$,

$$
\begin{aligned}
(\omega \cdot b)(X \cdot a) & =\omega\left(\left(\mathcal{R}_{1(1)}^{-1} \triangleright X\right) \cdot\left(\mathcal{R}_{1(2)}^{-1} \triangleright a\right)\right) \cdot\left(\mathcal{R}_{2}^{-1} \triangleright b\right) \\
& =\omega\left(\mathcal{R}_{1(1)}^{-1} \triangleright X\right) \cdot\left(\left(\mathcal{R}_{1}^{\prime-1} \mathcal{R}_{2}^{-1}\right) \triangleright b\right) \cdot\left(\left(\mathcal{R}_{2}^{\prime-1} \mathcal{R}_{1(2)}^{-1}\right) \triangleright a\right) \\
& =\omega\left(\mathcal{R}_{1}^{-1} \triangleright X\right) \cdot\left(\mathcal{R}_{2}^{-1} \triangleright b\right) \cdot a \\
& =((\omega \cdot b)(X)) \cdot a
\end{aligned}
$$

and

$$
\begin{aligned}
(\xi \triangleright \omega)(X \cdot a) & \left.=\xi_{(1)} \triangleright\left(\omega\left(\left(S \xi_{(2)}\right)_{(1)} \triangleright X\right) \cdot\left(S\left(\xi_{(2)}\right)_{(2)} \triangleright a\right)\right)\right) \\
& =\xi_{(1)} \triangleright\left(\omega\left(S\left(\xi_{(3)}\right) \triangleright X\right) \cdot\left(S\left(\xi_{(2)}\right) \triangleright a\right)\right) \\
& =\left(\xi_{(1)} \triangleright \omega\left(S\left(\xi_{(4)}\right) \triangleright X\right)\right) \cdot\left(\left(\xi_{(2)} S\left(\xi_{(3)}\right)\right) \triangleright a\right) \\
& =\left(\left(\xi_{(1)} \triangleright \omega\right)\left(\left(\xi_{(2)} S\left(\xi_{(3)}\right)\right) \triangleright X\right)\right) \cdot a \\
& =((\xi \triangleright \omega)(X)) \cdot a
\end{aligned}
$$

hold by the hexagon relations and the bialgebra anti-homomorphism properties of $S$. The $\mathcal{A}$-bimodule is $H$-equivariant, since

$$
\begin{aligned}
(\xi \triangleright(a \cdot \omega \cdot b))(X) & =\xi_{(1)} \triangleright\left((a \cdot \omega \cdot b)\left(S\left(\xi_{(2)}\right) \triangleright X\right)\right) \\
& =\left(\xi_{(1)} \triangleright a\right) \cdot\left(\xi_{(2)} \triangleright\left(\omega\left(\left(\mathcal{R}_{1}^{-1} S\left(\xi_{(4)}\right)\right) \triangleright X\right)\right)\right) \cdot\left(\left(\xi_{(3)} \mathcal{R}_{2}^{-1}\right) \triangleright b\right) \\
& =\left(\xi_{(1)} \triangleright a\right) \cdot\left(\left(\xi_{(2)} \triangleright \omega\right)\left(\left(\xi_{(3)} \mathcal{R}_{1}^{-1} S\left(\xi_{(5)}\right)\right) \triangleright X\right)\right) \cdot\left(\left(\xi_{(4)} \mathcal{R}_{2}^{-1}\right) \triangleright b\right) \\
& =\left(\xi_{(1)} \triangleright a\right) \cdot\left(\left(\xi_{(2)} \triangleright \omega\right)\left(\mathcal{R}_{1}^{-1} \triangleright X\right)\right) \cdot\left(\left(\mathcal{R}_{2}^{-1} \xi_{(3)}\right) \triangleright b\right) \\
& =\left(\left(\xi_{(1)} \triangleright a\right) \cdot\left(\xi_{(2)} \triangleright \omega\right) \cdot\left(\xi_{(3)} \triangleright b\right)\right)(X)
\end{aligned}
$$

and it is braided symmetric because

$$
\begin{aligned}
\left(\left(\mathcal{R}_{1}^{-1} \triangleright \omega\right) \cdot\left(\mathcal{R}_{2}^{-1} \triangleright a\right)\right)(X) & =\left(\left(\mathcal{R}_{1}^{-1} \triangleright \omega\right)\left(\mathcal{R}_{1}^{\prime-1} \triangleright X\right)\right) \cdot\left(\left(\mathcal{R}_{2}^{\prime}{ }^{-1} \mathcal{R}_{2}^{-1}\right) \triangleright a\right) \\
& =\left(\left(\mathcal{R}_{1}^{\prime \prime}-1 \mathcal{R}_{2}^{\prime-1} \mathcal{R}_{2}^{-1}\right) \triangleright a\right) \cdot\left(\mathcal{R}_{2}^{\prime \prime}{ }^{-1} \triangleright\left(\left(\mathcal{R}_{1}^{-1} \triangleright \omega\right)\left(\mathcal{R}_{1}^{\prime-1} \triangleright X\right)\right)\right) \\
& =(a \cdot \omega)(X) .
\end{aligned}
$$

These properties extend to the braided Graßmann algebra $\underline{\Omega}_{\mathcal{R}}^{\bullet}(\mathcal{A})$, giving an associative graded braided commutative product $\wedge_{\mathcal{R}}$. We further prove that $\mathrm{i}_{X}^{\mathcal{R}}$ is a graded braided derivation of the wedge product for $X \in \operatorname{Der}_{\mathcal{R}}(\mathcal{A})$. Let $\omega, \eta \in \underline{\Omega}_{\mathcal{R}}^{1}(\mathcal{A})$. Then

$$
\begin{aligned}
\mathrm{i}_{X}^{\mathcal{R}}\left(\omega \wedge_{\mathcal{R}} \eta\right)= & (-1)^{2-1}\left(\left(\mathcal{R}_{1(1)}^{-1} \triangleright \omega\right) \wedge_{\mathcal{R}}\left(\mathcal{R}_{1(2)}^{-1} \triangleright \eta\right)\right)\left(\cdot, \mathcal{R}_{2}^{-1} \triangleright X\right) \\
= & -\left(\mathcal{R}_{1(1)}^{-1} \triangleright \omega\right)\left(\mathcal{R}_{1(2)}^{-1} \triangleright \eta\right)\left(\mathcal{R}_{2}^{-1} \triangleright X\right) \\
& +\left(\mathcal{R}_{1(1)}^{-1} \triangleright \omega\right)\left(\left(\mathcal{R}_{1}^{\prime-1} \mathcal{R}_{2}^{-1}\right) \triangleright X\right)\left(\left(\mathcal{R}_{2}^{\prime-1} \mathcal{R}_{1(2)}^{-1}\right) \triangleright \eta\right) \\
= & \mathrm{i}_{X}^{\mathcal{R}}(\omega) \wedge_{\mathcal{R}} \eta+(-1)^{1 \cdot 1}\left(\mathcal{R}_{1}^{-1} \triangleright \omega\right) \wedge_{\mathcal{R}} \mathrm{i}_{\mathcal{R}_{2}^{-1}}^{\mathcal{R}} \triangleright X
\end{aligned}
$$

In particular this implies $\xi \triangleright\left(\mathrm{i}_{X}^{\mathcal{R}}\left(\omega \wedge_{\mathcal{R}} \eta\right)\right)=\mathrm{i}_{\xi_{(1)}^{\mathcal{R}} \triangleright X}\left(\left(\xi_{(2)} \triangleright \omega\right) \wedge_{\mathcal{R}}\left(\xi_{(3)} \triangleright \eta\right)\right)$ for all $\xi \in H$. Inductively, one shows

$$
\mathrm{i}_{X}^{\mathcal{R}}\left(\omega \wedge_{\mathcal{R}} \eta\right)=\left(\mathrm{i}_{X}^{\mathcal{R}} \omega\right) \wedge_{\mathcal{R}} \eta+(-1)^{k}\left(\mathcal{R}_{1}^{-1} \triangleright \omega\right) \wedge_{\mathcal{R}} \mathrm{i}_{\mathcal{R}_{2}^{-1} \triangleright X}^{\mathcal{R}} \eta
$$

and $\xi \triangleright\left(\mathrm{i}_{X}^{\mathcal{R}} \eta\right)=\mathrm{i}_{\xi_{(1)}^{\mathcal{R}} \triangleright X}\left(\xi_{(2)} \triangleright \eta\right)$ for all $\xi \in H, X \in \operatorname{Der}_{\mathcal{R}}(\mathcal{A}), \omega \in \underline{\Omega}_{\mathcal{R}}^{k}(\mathcal{A})$ and $\eta \in \underline{\Omega}_{\mathcal{R}}^{\bullet}(\mathcal{A})$. For factorizing elements $X_{1} \wedge_{\mathcal{R}} X_{2} \in \mathfrak{X}_{\mathcal{R}}^{2}(\mathcal{A})$ this implies

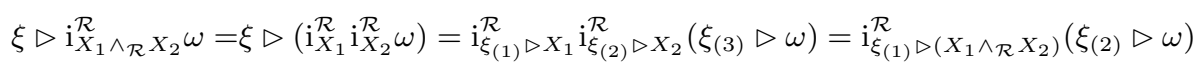


for all $\xi \in H$ and $\omega \in \underline{\Omega}_{\mathcal{R}}^{\bullet}(\mathcal{A})$ and inductively one obtains $\xi \triangleright\left(\mathrm{i}_{X}^{\mathcal{R}} \omega\right)=\mathrm{i}_{\xi_{(1)}}^{\mathcal{R}} \triangleright X\left(\xi_{(2)} \triangleright \omega\right)$ for any $X \in \underline{\Omega}_{\mathcal{R}}^{\bullet}(\mathcal{A})$. It is easy to verify that $\mathrm{i}^{\mathcal{R}}$ sarisfies the linearity properties

$$
\begin{aligned}
& \mathrm{i}_{a \cdot X}^{\mathcal{R}} \omega=a \cdot\left(\mathrm{i}_{X}^{\mathcal{R}} \omega\right), \mathrm{i}_{X \cdot a}^{\mathcal{R}} \omega=\left(\mathrm{i}_{X}^{\mathcal{R}}\left(\mathcal{R}_{1}^{-1} \triangleright \omega\right)\right) \cdot\left(\mathcal{R}_{2}^{-1} \triangleright a\right), \\
& \mathrm{i}_{X}^{\mathcal{R}}(\omega \cdot a)=\left(\mathrm{i}_{X}^{\mathcal{R}} \omega\right) \cdot a, \mathrm{i}_{X}^{\mathcal{R}}(a \cdot \omega)=\left(\mathcal{R}_{1}^{-1} \triangleright a\right) \cdot\left(\mathrm{i}_{\mathcal{R}_{2}^{-1}}^{\mathcal{R}} \triangleright X\right. \\
&
\end{aligned}
$$

for all $X \in \mathfrak{X}_{\mathcal{R}}^{\bullet}(\mathcal{A}), a \in \mathcal{A}$ and $\omega \in \underline{\Omega}_{\mathcal{R}}^{\bullet}(\mathcal{A})$. This concludes the proof of the lemma.

\subsection{Braided Cartan Calculi and Gauge Equivalence}

In the following pages we construct a noncommutative Cartan calculus for any braided symmetric algebra. The development is entirely parallel to the Cartan calculus of a commutative algebra, however in a symmetric braided monoidal category. In particular, we are not constrained to use the center of the algebra. Afterwards we define a twist deformation of any braided Cartan calculus and show that it is isomorphic to the braided Cartan calculus of the twist deformed algebra with respect to the twisted triangular structure.

One defines a differential $\mathrm{d}: \underline{\Omega}_{\mathcal{R}}^{\bullet}(\mathcal{A}) \rightarrow \underline{\Omega}_{\mathcal{R}}^{\bullet+1}(\mathcal{A})$ on $a \in \mathcal{A}$ by i $\mathrm{i}_{X}^{\mathcal{R}}(\mathrm{d} a)=X(a)$ for all $X \in \operatorname{Der}_{\mathcal{R}}(\mathcal{A})$, on $\omega \in \underline{\Omega}_{\mathcal{R}}^{1}(\mathcal{A})$ by

$$
(\mathrm{d} \omega)(X, Y)=\left(\mathcal{R}_{1}^{-1} \triangleright X\right)\left(\left(\mathcal{R}_{2}^{-1} \triangleright \omega\right)(Y)\right)-\left(\mathcal{R}_{1}^{-1} \triangleright Y\right)\left(\mathcal{R}_{2}^{-1} \triangleright(\omega(X))\right)-\omega\left([X, Y]_{\mathcal{R}}\right)
$$

for all $X, Y \in \operatorname{Der}_{\mathcal{R}}(\mathcal{A})$ and extends d to higher wedge powers by demanding it to be a graded derivation with respect to $\wedge_{\mathcal{R}}$, i.e.

$$
\mathrm{d}\left(\omega_{1} \wedge_{\mathcal{R}} \omega_{2}\right)=\left(\mathrm{d} \omega_{1}\right) \wedge_{\mathcal{R}} \omega_{2}+(-1)^{k} \omega_{1} \wedge_{\mathcal{R}}\left(\mathrm{d} \omega_{2}\right)
$$

for $\omega_{1} \in \underline{\Omega}_{\mathcal{R}}^{k}(\mathcal{A})$ and $\omega_{2} \in \underline{\Omega}_{\mathcal{R}}^{\bullet}(\mathcal{A})$. Alternatively we can directly define $\mathrm{d} \omega \in \underline{\Omega}_{\mathcal{R}}^{k+1}(\mathcal{A})$ for any $\omega \in \underline{\Omega}_{\mathcal{R}}^{k}(\overline{\mathcal{A}})$ by

$$
\begin{aligned}
(\mathrm{d} \omega)\left(X_{0}, \ldots, X_{k}\right)= & \sum_{i=0}^{k}(-1)^{i}\left(\mathcal{R}_{1}^{-1} \triangleright X_{i}\right)\left(\left(\mathcal{R}_{2(1)}^{-1} \triangleright \omega\right)(\right. \\
& \left.\left.\mathcal{R}_{2(2)}^{-1} \triangleright X_{0}, \ldots, \mathcal{R}_{2(i+1)}^{-1} \triangleright X_{i-1}, \widehat{X}_{i}, X_{i+1}, \ldots, X_{k}\right)\right) \\
& +\sum_{i<j}(-1)^{i+j} \omega\left(\left[\mathcal{R}_{1}^{-1} \triangleright X_{i}, \mathcal{R}_{1}^{\prime-1} \triangleright X_{j}\right]_{\mathcal{R}},\right. \\
& \left(\mathcal{R}_{2(1)}^{\prime}-1 \mathcal{R}_{2(1)}^{-1}\right) \triangleright X_{0}, \ldots,\left(\mathcal{R}_{2(i)}^{\prime-1} \mathcal{R}_{2(i)}^{-1}\right) \triangleright X_{i-1}, \widehat{X}_{i}, \\
& \left.\mathcal{R}_{2(i+1)}^{\prime-1} \triangleright X_{i+1}, \ldots, \mathcal{R}_{2(j-1)}^{\prime} \triangleright X_{j-1}, \widehat{X_{j}}, X_{j+1}, \ldots, X_{k}\right)
\end{aligned}
$$

for all $X_{0}, \ldots, X_{k} \in \operatorname{Der}_{\mathcal{R}}(\mathcal{A})$. It is sufficient to prove $\mathrm{d}^{2}=0$ on $\underline{\Omega}_{\mathcal{R}}^{k}(\mathcal{A})$ for $k<2$, since $\mathrm{d}^{2}$ is a graded braided derivation. The computations can be found in [41. Define now the braided differential forms $\Omega_{\mathcal{R}}^{\bullet}(\mathcal{A})$ on $\mathcal{A}$ to be the smallest differential graded subalgebra of $\underline{\Omega}_{\mathcal{R}}^{\bullet}(\mathcal{A})$ such that $\mathcal{A} \subseteq \Omega_{\mathcal{R}}(\mathcal{A})$. Every element of $\Omega_{\mathcal{R}}^{k}(\mathcal{A})$ can be written as a finite sum of elements of the form $a_{0} \mathrm{~d} a_{1} \wedge_{\mathcal{R}} \ldots \wedge_{\mathcal{R}} \mathrm{d} a_{k}$, where $a_{0}, \ldots, a_{k} \in \mathcal{A}$. Using eq.(3.34) and the fact that the braided commutator is $H$-equivariant it immediately follows that d commutes with the left $H$-module action. In other words, $\mathrm{d}$ is equivariant with respect to the adjoint action, implying

$$
(\xi \triangleright \mathrm{d}) \omega=\xi_{(1)} \triangleright\left(\mathrm{d}\left(S\left(\xi_{(2)}\right) \triangleright \omega\right)\right)=\left(\xi_{(1)} S\left(\xi_{(2)}\right)\right) \triangleright(\mathrm{d} \omega)=\epsilon(\xi) \mathrm{d} \omega
$$

for all $\xi \in H$ and $\omega \in \Omega_{\mathcal{R}}^{\bullet}(\mathcal{A})$. Recall that the graded braided commutator of two homogeneous maps $\Phi, \Psi: \mathfrak{G}^{\bullet} \rightarrow \mathfrak{G}^{\bullet}$ of degree $k$ and $\ell$ between braided Graßmann algebras is defined by

$$
[\Phi, \Psi]_{\mathcal{R}}=\Phi \circ \Psi-(-1)^{k \ell}\left(\mathcal{R}_{1}^{-1} \triangleright \Psi\right) \circ\left(\mathcal{R}_{2}^{-1} \triangleright \Phi\right) .
$$

If $\Phi$ or $\Psi$ is equivariant, the graded braided commutator coincides with the graded commutator. Furthermore, if $\Phi, \Psi: \mathfrak{X}_{\mathcal{R}}^{\bullet}(\mathcal{A}) \otimes \mathfrak{G}^{\bullet} \rightarrow \mathfrak{G}^{\bullet}$ are $H$-equivariant maps such that $\Phi_{X}, \Psi_{Y}: \mathfrak{G}^{\bullet} \rightarrow \mathfrak{G}^{\bullet}$ are homogeneous of degree $k$ and $\ell$ for any $X \in \mathfrak{X}_{\mathcal{R}}^{k}(\mathcal{A})$ and $Y \in \mathfrak{X}_{\mathcal{R}}^{\ell}(\mathcal{A})$, respectively, the graded braided commutator of $\Phi_{X}$ and $\Psi_{Y}$ reads

$$
\left[\Phi_{X}, \Psi_{Y}\right]_{\mathcal{R}}=\Phi_{X} \circ \Psi_{Y}-(-1)^{k \ell} \Psi_{\mathcal{R}_{1}^{-1} \triangleright Y} \circ \Phi_{\mathcal{R}_{2}^{-1} \triangleright X} .
$$

For any $X \in \mathfrak{X}_{\mathcal{R}}^{\bullet}(\mathcal{A})$ we define the braided Lie derivative $\mathscr{L}^{\mathcal{R}}: \mathfrak{X}_{\mathcal{R}}^{\bullet}(\mathcal{A}) \otimes \Omega_{\mathcal{R}}^{\bullet}(\mathcal{A}) \rightarrow \Omega_{\mathcal{R}}^{\bullet}(\mathcal{A})$ by $\mathscr{L}_{X}^{\mathcal{R}}=\left[\mathrm{i}_{X}^{\mathcal{R}}, \mathrm{d}\right]_{\mathcal{R}}$. It is $H$-equivariant and if $X \in \mathfrak{X}_{\mathcal{R}}^{k}(\mathcal{A}), \mathscr{L}_{X}^{\mathcal{R}}$ is a homogeneous map of degree $-(k-1)$. For $k=1$ we obtain a braided derivation $\mathscr{L}_{X}^{\mathcal{R}}$ of $\Omega_{\mathcal{R}}^{\bullet}(\mathcal{A})$. 
Lemma 3.5. One has

$$
\mathscr{L}_{a}^{\mathcal{R}} \omega=-(\mathrm{d} a) \wedge_{\mathcal{R}} \omega \text { and } \mathscr{L}_{X}^{\mathcal{R}} \wedge_{\mathcal{R}} Y=\mathrm{i}_{X}^{\mathcal{R}} \mathscr{L}_{Y}^{\mathcal{R}}+(-1)^{\ell} \mathscr{L}_{X}^{\mathcal{R}} \mathrm{i}_{Y}^{\mathcal{R}}
$$

for all $a \in \mathcal{A}, \omega \in \Omega_{\mathcal{R}}^{\bullet}(\mathcal{A}), X \in \mathfrak{X}_{\mathcal{R}}^{\bullet}(\mathcal{A})$ and $Y \in \mathfrak{X}_{\mathcal{R}}^{\ell}(\mathcal{A})$. If $X, Y \in \mathfrak{X}_{\mathcal{R}}^{1}(\mathcal{A})$

$$
\left[\mathscr{L}_{X}^{\mathcal{R}}, \mathrm{i}_{Y}^{\mathcal{R}}\right]_{\mathcal{R}}=\mathrm{i}_{[X, Y]_{\mathcal{R}}}^{\mathcal{R}}
$$

holds.

Proof. By the definition of the braided Lie derivative

$$
\mathscr{L}_{a}^{\mathcal{R}} \omega=\mathrm{i}_{a}^{\mathcal{R}} \mathrm{d} \omega-(-1)^{0 \cdot 1} \mathrm{~d}\left(\mathrm{i}_{a}^{\mathcal{R}} \omega\right)=a \wedge_{\mathcal{R}} \mathrm{d} \omega-\left((\mathrm{d} a) \wedge_{\mathcal{R}} \omega+(-1)^{0} a \wedge_{\mathcal{R}} \mathrm{d} \omega\right)=-(\mathrm{d} a) \wedge_{\mathcal{R}} \omega
$$

follows. From the graded braided Leibniz rule of the graded braided commutator we obtain

$$
\begin{aligned}
\mathscr{L}_{X}^{\mathcal{R}} \wedge_{\mathcal{R}} Y & =\left[\mathrm{i}_{X}^{\mathcal{R}} \wedge_{\mathcal{R} Y}, \mathrm{~d}\right]_{\mathcal{R}}=\left[\mathrm{i}_{X}^{\mathcal{R}} \mathrm{i}_{Y}^{\mathcal{R}}, \mathrm{d}\right]_{\mathcal{R}}=\mathrm{i}_{X}^{\mathcal{R}}\left[\mathrm{i}_{Y}^{\mathcal{R}}, \mathrm{d}\right]_{\mathcal{R}}+(-1)^{-1 \cdot \ell}\left[\mathrm{i}_{X}^{\mathcal{R}}, \mathrm{d}\right]_{\mathcal{R}} \mathrm{i}_{Y}^{\mathcal{R}} \\
& =\mathrm{i}_{X}^{\mathcal{R}} \mathscr{L}_{Y}^{\mathcal{R}}+(-1)^{\ell} \mathscr{L}_{X}^{\mathcal{R}} \mathrm{i}_{Y}^{\mathcal{R}} .
\end{aligned}
$$

The missing formula trivially holds on braided differential forms of degree 0 , while for $\omega \in$ $\Omega_{\mathcal{R}}^{1}(\mathcal{A})$ one obtains

$$
\begin{aligned}
& {\left[\mathscr{L}_{X}^{\mathcal{R}}, \mathrm{i}_{Y}^{\mathcal{R}}\right]_{\mathcal{R}} \omega=\mathscr{L}_{X}^{\mathcal{R}} \mathrm{i}_{Y}^{\mathcal{R}} \omega-(-1)^{0 \cdot 1} \mathrm{i}_{\mathcal{R}_{1}^{-1} \triangleright Y}^{\mathcal{R}} \mathscr{L}_{\mathcal{R}_{2}^{-1} \triangleright X}^{\mathcal{R}} \omega} \\
& =\left(\mathrm{i}_{X}^{\mathcal{R}} \mathrm{d}+\mathrm{di}_{X}^{\mathcal{R}}\right) \mathrm{i}_{Y}^{\mathcal{R}} \omega-\mathrm{i}_{\mathcal{R}_{1}^{-1} \triangleright Y}^{\mathcal{R}}\left(\mathrm{i}_{\mathcal{R}_{2}^{-1} \triangleright X}^{\mathcal{R}} \mathrm{d}+\mathrm{di}_{\mathcal{R}_{2}^{-1} \triangleright X}^{\mathcal{R}}\right) \omega \\
& =X\left(\mathrm{i}_{Y}^{\mathcal{R}} \omega\right)+0+\left(\mathrm{d}\left(\left(\mathcal{R}_{1}^{\prime \prime}{ }^{-1} \mathcal{R}_{1}^{\prime-1}\right) \triangleright \omega\right)\right)\left(\left(\mathcal{R}_{2}^{\prime \prime}{ }^{-1} \mathcal{R}_{1}^{-1}\right) \triangleright Y,\left(\mathcal{R}_{2}^{\prime-1} \mathcal{R}_{2}^{-1}\right) \triangleright X\right) \\
& -\left(\mathcal{R}_{1}^{-1} \triangleright Y\right)\left(\mathrm{i}_{\mathcal{R}_{2}^{-1} \triangleright X}^{\mathcal{R}} \omega\right) \\
& =\mathrm{i}_{[X, Y]}^{\mathcal{R}} \omega
\end{aligned}
$$

for all $X, Y \in \mathfrak{X}_{\mathcal{R}}^{1}(\mathcal{A})$. Since $\left[\mathscr{L}_{X}^{\mathcal{R}}, \mathrm{i}_{Y}^{\mathcal{R}}\right]_{\mathcal{R}}$ is a graded braided derivation this is all we have to prove.

We are prepared to prove the main theorem of this section. It assigns to any braided commutative left $H$-module algebra $\mathcal{A}$ a noncommutative Cartan calculus, which we call the braided Cartan calculus of $\mathcal{A}$ in the following.

Theorem 3.6 (Braided Cartan calculus). Let $\mathcal{A}$ be a braided commutative left $H$-module algebra and consider the braided differential forms $\left(\Omega_{\mathcal{R}}(\mathcal{A}), \wedge_{\mathcal{R}}, \mathrm{d}\right)$ and braided multivector fields $\left(\mathfrak{X}_{\mathcal{R}}^{\bullet}(\mathcal{A}), \wedge_{\mathcal{R}}, \llbracket \cdot, \cdot \rrbracket_{\mathcal{R}}\right)$ on $\mathcal{A}$. The homogeneous maps

$$
\mathscr{L}_{X}^{\mathcal{R}}: \Omega_{\mathcal{R}}^{\bullet}(\mathcal{A}) \rightarrow \Omega_{\mathcal{R}}^{\bullet-(k-1)}(\mathcal{A}) \text { and } \mathrm{i}_{X}^{\mathcal{R}}: \Omega_{\mathcal{R}}^{\bullet}(\mathcal{A}) \rightarrow \Omega_{\mathcal{R}}^{\bullet-k}(\mathcal{A}),
$$

where $X \in \mathfrak{X}_{\mathcal{R}}^{k}(\mathcal{A})$, and $\mathrm{d}: \Omega_{\mathcal{R}}^{\bullet}(\mathcal{A}) \rightarrow \Omega_{\mathcal{R}}^{\bullet+1}(\mathcal{A})$ satisfy

$$
\begin{aligned}
{\left[\mathscr{L}_{X}^{\mathcal{R}}, \mathscr{L}_{Y}^{\mathcal{R}}\right]_{\mathcal{R}} } & =\mathscr{L}_{\llbracket X, Y \rrbracket_{\mathcal{R}}}^{\mathcal{R}}, & {\left[\mathrm{i}_{X}^{\mathcal{R}}, \mathrm{i}_{Y}^{\mathcal{R}}\right]_{\mathcal{R}} } & =0, \\
{\left[\mathscr{L}_{X}^{\mathcal{R}}, \mathrm{i}_{Y}^{\mathcal{R}}\right]_{\mathcal{R}} } & =\mathrm{i}_{\llbracket X, Y \rrbracket_{\mathcal{R}}}^{\mathcal{R}}, & {\left[\mathrm{i}_{X}^{\mathcal{R}}, \mathrm{d}\right]_{\mathcal{R}} } & =\mathscr{L}_{X}^{\mathcal{R}}, \\
{\left[\mathscr{L}_{X}^{\mathcal{R}}, \mathrm{d}\right]_{\mathcal{R}} } & =0, & {[\mathrm{~d}, \mathrm{~d}]_{\mathcal{R}} } & =0,
\end{aligned}
$$

for all $X, Y \in \mathfrak{X}_{\mathcal{R}}^{\bullet}(\mathcal{A})$.

Proof. We are going to prove the above formulas in reversed order. Since d is a differential it follows that $[\mathrm{d}, \mathrm{d}]_{\mathcal{R}}=2 \mathrm{~d}^{2}=0$. Recall that there is no braiding appearing here since $\mathrm{d}$ is equivariant. By the definition of the braided Lie derivative $\left[\mathrm{i}_{X}^{\mathcal{R}}, \mathrm{d}\right]_{\mathcal{R}}=\mathscr{L}_{X}^{\mathcal{R}}$ holds for all $X \in \mathfrak{X}_{\mathcal{R}}^{\bullet}(\mathcal{A})$. Let $X \in \mathfrak{X}_{\mathcal{R}}^{k}(\mathcal{A})$ and $Y \in \mathfrak{X}_{\mathcal{R}}^{\ell}(\mathcal{A})$. Then

$$
\left[\mathrm{i}_{X}^{\mathcal{R}}, \mathrm{i}_{Y}^{\mathcal{R}}\right]_{\mathcal{R}}=\mathrm{i}_{X}^{\mathcal{R}} i_{Y}^{\mathcal{R}}-(-1)^{k \ell} \mathrm{i}_{\mathcal{R}_{1}^{-1} \triangleright Y}^{\mathcal{R}} \mathrm{i}_{\mathcal{R}_{2}^{-1} \triangleright X}^{\mathcal{R}}=\mathrm{i}_{X \wedge_{\mathcal{R}} Y-(-1)^{k \ell}\left(\mathcal{R}_{1}^{-1} \triangleright Y\right) \wedge_{\mathcal{R}}\left(\mathcal{R}_{2}^{-1} \triangleright X\right)}^{\mathcal{R}}=0
$$

follows from the definition of $\mathrm{i}_{X \wedge_{\mathcal{R}} Y}^{\mathcal{R}}=\mathrm{i}_{X}^{\mathcal{R}} \mathrm{i}_{Y}^{\mathcal{R}}$. Using the graded braided Jacobi identity of the graded braided commutator we obtain

$$
\left[\left[\mathrm{i}_{X}^{\mathcal{R}}, \mathrm{d}\right]_{\mathcal{R}}, \mathrm{d}\right]_{\mathcal{R}}=\left[\mathrm{i}_{X}^{\mathcal{R}},[\mathrm{d}, \mathrm{d}]_{\mathcal{R}}\right]_{\mathcal{R}}+(-1)^{1 \cdot 1}\left[\left[\mathrm{i}_{X}^{\mathcal{R}}, \mathrm{d}\right]_{\mathcal{R}}, \mathrm{d}\right]_{\mathcal{R}}=-\left[\left[\mathrm{i}_{X}^{\mathcal{R}}, \mathrm{d}\right]_{\mathcal{R}}, \mathrm{d}\right]_{\mathcal{R}}
$$

for all $X \in \mathfrak{X}_{\mathcal{R}}^{\bullet}(\mathcal{A})$, which implies $\left[\mathscr{L}_{X}^{\mathcal{R}}, \mathrm{d}\right]_{\mathcal{R}}=0$. Again, there is no braiding appearing since $\mathrm{d}$ is equivariant. Recall that the braided Schouten-Nijenhuis bracket of a homogeneous element $Y=Y_{1} \wedge_{\mathcal{R}} \cdots \wedge_{\mathcal{R}} Y_{\ell} \in \mathfrak{X}_{\mathcal{R}}^{\ell}(\mathcal{A})$ with $a \in \mathcal{A}$ and $X \in \mathfrak{X}_{\mathcal{R}}^{1}(\mathcal{A})$ read

$\llbracket a, Y \rrbracket_{\mathcal{R}}=\sum_{j=1}^{\ell}(-1)^{j+1}\left(\mathcal{R}_{1(1)}^{-1} \triangleright Y_{1}\right) \wedge_{\mathcal{R}} \cdots \wedge_{\mathcal{R}}\left(\mathcal{R}_{1(j-1)}^{-1} \triangleright Y_{j-1}\right) \wedge_{\mathcal{R}} \llbracket \mathcal{R}_{2}^{-1} \triangleright a, Y_{j} \rrbracket_{\mathcal{R}} \wedge_{\mathcal{R}} Y_{j+1} \wedge_{\mathcal{R}} \cdots \wedge_{\mathcal{R}} Y_{\ell}$ 
and

$$
\llbracket X, Y \rrbracket_{\mathcal{R}}=\sum_{j=1}^{\ell}\left(\mathcal{R}_{1(1)}^{-1} \triangleright Y_{1}\right) \wedge_{\mathcal{R}} \cdots \wedge_{\mathcal{R}}\left(\mathcal{R}_{1(j-1)}^{-1} \triangleright Y_{j-1}\right) \wedge_{\mathcal{R}}\left[\mathcal{R}_{2}^{-1} \triangleright X, Y_{j}\right]_{\mathcal{R}} \wedge_{\mathcal{R}} Y_{j+1} \wedge_{\mathcal{R}} \cdots \wedge_{\mathcal{R}} Y_{\ell}
$$

respectively. If $\ell=1$ we obtain

$$
\begin{aligned}
{\left[\mathscr{L}_{a}^{\mathcal{R}}, \mathrm{i}_{Y}^{\mathcal{R}}\right]_{\mathcal{R}} \omega } & =\left(\mathscr{L}_{a}^{\mathcal{R}} \mathrm{i}_{Y}^{\mathcal{R}}-(-1)^{(-1) \cdot 1} \mathrm{i}_{\mathcal{R}_{1}^{-1} \triangleright Y}^{\mathcal{R}} \mathscr{L}_{\mathcal{R}_{2}^{-1} \triangleright a}^{\mathcal{R}}\right) \omega \\
& =-\mathrm{d} a \wedge_{\mathcal{R}} \mathrm{i}_{Y}^{\mathcal{R}} \omega-\mathrm{i}_{\mathcal{R}_{1}^{\mathcal{R}} \triangleright Y}^{\mathcal{R}}\left(\mathrm{d}\left(\mathcal{R}_{2}^{-1} \triangleright a\right) \wedge_{\mathcal{R}} \omega\right) \\
& =-\mathrm{d} a \wedge_{\mathcal{R}} \mathrm{i}_{Y}^{\mathcal{R}} \omega-\left(\mathcal{R}_{1}^{-1} \triangleright Y\right)\left(\mathcal{R}_{2}^{-1} \triangleright a\right) \cdot \omega+\mathrm{d}\left(\left(\mathcal{R}_{1}^{\prime-1} \mathcal{R}_{2}^{-1}\right) \triangleright a\right) \wedge_{\mathcal{R}} \mathrm{i}_{\left(\mathcal{R}_{2}^{\prime-1} \mathcal{R}_{1}^{-1}\right) \triangleright Y}^{\mathcal{R}} \omega \\
& =\mathrm{i}_{\llbracket a, Y \rrbracket_{\mathcal{R}}}^{\mathcal{R}} \omega
\end{aligned}
$$

for all $\omega \in \Omega_{\mathcal{R}}^{\bullet}(\mathcal{A})$ by Lemma 3.5 Using the graded braided Leibniz rule this extends to any $\ell>1$, namely

$$
\begin{aligned}
& {\left[\mathscr{L}_{a}^{\mathcal{R}}, \mathrm{i}_{Y_{1} \wedge_{\mathcal{R}} \cdots \wedge_{\mathcal{R}} Y_{\ell}}^{\mathcal{R}}\right]_{\mathcal{R}}=\left[\mathscr{L}_{a}^{\mathcal{R}}, \mathrm{i}_{Y_{1}}^{\mathcal{R}}\right]_{\mathcal{R}} \mathrm{i}_{Y_{2} \wedge_{\mathcal{R}} \cdots \wedge_{\mathcal{R}} Y_{\ell}}^{\mathcal{R}}+(-1)^{(-1) \cdot 1^{\mathcal{R}}} \mathrm{i}_{\mathcal{R}_{1}^{-1} \triangleright Y_{1}}\left[\mathscr{L}_{\mathcal{R}_{2}^{-1} \triangleright a}^{\mathcal{R}}, \mathrm{i}_{Y_{2} \wedge_{\mathcal{R}} \cdots \wedge_{\mathcal{R}} Y_{\ell}}^{\mathcal{R}}\right]} \\
& =\mathrm{i}_{\llbracket a, Y_{1} \rrbracket_{\mathcal{R}} \wedge_{\mathcal{R}} Y_{2} \wedge_{\mathcal{R}} \cdots \wedge_{\mathcal{R}} Y_{\ell}}-\mathrm{i}_{\mathcal{R}_{1}^{-1} \triangleright Y_{1}}^{\mathcal{R}}\left[\mathscr{L}_{\mathcal{R}_{2}^{-1} \triangleright a}^{\mathcal{R}}, \mathrm{i}_{Y_{2} \wedge_{\mathcal{R}} \cdots \wedge_{\mathcal{R}} Y_{\ell}}^{\mathcal{R}}\right] \\
& =\cdots=\mathrm{i}_{\llbracket a, Y \rrbracket_{\mathcal{R}}}^{\mathcal{R}} .
\end{aligned}
$$

Again by Lemma 3.5 we know that $\left[\mathscr{L}_{X}^{\mathcal{R}}, \mathrm{i}_{Y}^{\mathcal{R}}\right]_{\mathcal{R}}=\mathrm{i}_{[X, Y]_{\mathcal{R}}}^{\mathcal{R}}$ holds for $\ell=1$ and $X \in \mathfrak{X}_{\mathcal{R}}^{1}(\mathcal{A})$. Using the graded braided Leibniz rule this extends to all $Y \in \mathfrak{X}_{\mathcal{R}}^{\bullet}(\mathcal{A})$. Assume now that $\left[\mathscr{L}_{X}^{\mathcal{R}}, \mathrm{i}_{Z}^{\mathcal{R}}\right]_{\mathcal{R}}=\mathrm{i}_{\llbracket X, Z \rrbracket_{\mathcal{R}}}^{\mathcal{R}}$ holds for all $X \in \mathfrak{X}_{\mathcal{R}}^{k}(\mathcal{A})$ and $Z \in \mathfrak{X}_{\mathcal{R}}^{\bullet}(\mathcal{A})$ for a fixed $k>0$. Then, for all $X \in \mathfrak{X}_{\mathcal{R}}^{k}(\mathcal{A}), Y \in \mathfrak{X}_{\mathcal{R}}^{1}(\mathcal{A})$ and $Z \in \mathfrak{X}_{\mathcal{R}}^{m}(\mathcal{A})$ it follows that

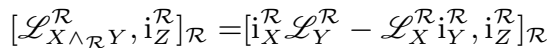

$$
\begin{aligned}
& =\mathrm{i}_{X}^{\mathcal{R}}\left[\mathscr{L}_{Y}^{\mathcal{R}}, \mathrm{i}_{Z}^{\mathcal{R}}\right]_{\mathcal{R}}+\left[\mathrm{i}_{X}^{\mathcal{R}}, \mathrm{i}_{\mathcal{R}_{1}^{-1} \triangleright Z}^{\mathcal{R}}\right]_{\mathcal{R}} \mathscr{L}_{\mathcal{R}_{2}^{-1} \triangleright Y}^{\mathcal{R}} \\
& -\mathscr{L}_{X}^{\mathcal{R}}\left[\mathrm{i}_{Y}^{\mathcal{R}}, \mathrm{i}_{Z}^{\mathcal{R}}\right]_{\mathcal{R}}-(-1)^{m}\left[\mathscr{L}_{X}^{\mathcal{R}}, \mathrm{i}_{\mathcal{R}_{1}^{-1} \triangleright Z}^{\mathcal{R}}\right]_{\mathcal{R}} \mathrm{i}_{\mathcal{R}_{2}^{-1} \triangleright Y}^{\mathcal{R}} \\
& =\mathrm{i}_{X}^{\mathcal{R}}\left[\mathscr{L}_{Y}^{\mathcal{R}}, \mathrm{i}_{Z}^{\mathcal{R}}\right]_{\mathcal{R}}-(-1)^{m}\left[\mathscr{L}_{X}^{\mathcal{R}}, \mathrm{i}_{\mathcal{R}_{1}^{-1} \triangleright Z}^{\mathcal{R}}\right]_{\mathcal{R}} \mathrm{i}_{\mathcal{R}_{2}^{-1} \triangleright Y}^{\mathcal{R}} \\
& =\mathrm{i}_{X}^{\mathcal{R}} \mathrm{i}_{\llbracket Y, Z \rrbracket_{\mathcal{R}}}^{\mathcal{R}}-(-1)^{m_{1}} \mathrm{i}_{\llbracket X, \mathcal{R}_{1}^{-1} \triangleright Z \rrbracket_{\mathcal{R}}} \mathrm{i}_{\left(\mathcal{R}_{2}^{-1} \triangleright Y\right)}^{\mathcal{R}} \\
& =\mathrm{i}_{X \wedge_{\mathcal{R}} \llbracket Y, Z \rrbracket_{\mathcal{R}}}^{\mathcal{R}}+(-1)^{m-1} \dot{i}_{\llbracket X, \mathcal{R}_{1}^{-1} \triangleright Z \rrbracket_{\mathcal{R}} \wedge_{\mathcal{R}}\left(\mathcal{R}_{2}^{-1} \triangleright Y\right)} \\
& =\mathrm{i}_{\llbracket X \wedge_{\mathcal{R}}^{\mathcal{R}} Y, Z \rrbracket_{\mathcal{R}}}
\end{aligned}
$$

for all $X \in \mathfrak{X}_{\mathcal{R}}^{k}(\mathcal{A}), Y \in \mathfrak{X}_{\mathcal{R}}^{1}(\mathcal{A})$ and $Z \in \mathfrak{X}_{\mathcal{R}}^{m}(\mathcal{A})$ using Lemma 3.5 By induction $\left[\mathscr{L}_{X}^{\mathcal{R}}, \mathrm{i}_{Y}^{\mathcal{R}}\right]_{\mathcal{R}}=$ $\mathrm{i}_{\llbracket X, Y \rrbracket_{\mathcal{R}}}^{\mathcal{R}}$ for all $X, Y \in \mathfrak{X}_{\mathcal{R}}^{\bullet}(\mathcal{A})$. The remaining formula is verified via

$$
\begin{aligned}
{\left[\mathscr{L}_{X}^{\mathcal{R}}, \mathscr{L}_{Y}^{\mathcal{R}}\right]_{\mathcal{R}} } & =\left[\mathscr{L}_{X}^{\mathcal{R}},\left[\mathrm{i}_{Y}^{\mathcal{R}}, \mathrm{d}\right]_{\mathcal{R}}\right]_{\mathcal{R}} \\
& =\left[\left[\mathscr{L}_{X}^{\mathcal{R}}, \mathrm{i}_{Y}^{\mathcal{R}}\right]_{\mathcal{R}}, \mathrm{d}\right]_{\mathcal{R}}+(-1)^{(k-1) \ell}\left[\mathrm{i}_{\mathcal{R}_{1}^{\mathcal{R}} \triangleright Y},\left[\mathscr{L}_{\left.\left.\mathcal{R}_{2}^{\mathcal{R}} \triangleright{ }^{-1}, \mathrm{~d}\right]_{\mathcal{R}}\right]_{\mathcal{R}}}\right.\right. \\
& =\left[\mathrm{i}_{\llbracket X, Y}^{\mathcal{R}} \rrbracket_{\mathcal{R}}, \mathrm{d}\right]_{\mathcal{R}}+0 \\
& =\mathscr{L}_{\llbracket X, Y}^{\mathcal{R}} \rrbracket_{\mathcal{R}}
\end{aligned}
$$

for all $X \in \mathfrak{X}_{\mathcal{R}}^{k}(\mathcal{A})$ and $Y \in \mathfrak{X}_{\mathcal{R}}^{\ell}(\mathcal{A})$. This concludes the proof of the theorem.

In particular, the Cartan calculus on a commutative algebra is a braided Cartan calculus with respect to the trivial triangular structure and a (possibly trivial) action of a cocommutative Hopf algebra. We discuss a further class of examples which is to some extent already present in the literature, see [5] for $\mathcal{R}=1 \otimes 1$ and [7] Proposition 3.22. for the first order calculus in the case of a quasi-triangular Hopf algebra and non-associative algebras. Fix a triangular Hopf algebra $(H, \mathcal{R})$, a braided commutative left $H$-module algebra $\mathcal{A}$ and a Drinfel'd twist $\mathcal{F}$ on $H$ in the following. Recall from Theorem 2.11 that the Drinfel'd functor

$$
\operatorname{Drin}_{\mathcal{F}}:\left({ }_{\mathcal{A}}^{H} \mathcal{M}_{\mathcal{A}}^{\mathcal{R}}, \otimes_{\mathcal{A}}, c^{\mathcal{R}}\right) \rightarrow\left({ }_{\mathcal{A}_{\mathcal{F}}}^{H_{\mathcal{F}}} \mathcal{M}_{\mathcal{A}_{\mathcal{F}}}^{\mathcal{R}_{\mathcal{F}}}, \otimes_{\mathcal{A}_{\mathcal{F}}}, c^{\mathcal{R}_{\mathcal{F}}}\right)
$$

is a braided monoidal equivalence of braided monoidal categories with braided monoidal natural transformation given on objects $\mathcal{M}$ and $\mathcal{M}^{\prime}$ of ${ }_{\mathcal{A}}^{H} \mathcal{M}_{\mathcal{A}}^{\mathcal{R}}$ by

$$
\varphi_{\mathcal{M}, \mathcal{M}^{\prime}}: \mathcal{M}_{\mathcal{F}} \otimes_{\mathcal{A}_{\mathcal{F}}} \mathcal{M}_{\mathcal{F}}^{\prime} \ni\left(m \otimes_{\mathcal{A}_{\mathcal{F}}} m^{\prime}\right) \mapsto\left(\mathcal{F}_{1}^{-1} \triangleright m\right) \otimes_{\mathcal{A}}\left(\mathcal{F}_{2}^{-1} \triangleright m^{\prime}\right) \in\left(\mathcal{M} \otimes_{\mathcal{A}} \mathcal{M}^{\prime}\right)_{\mathcal{F}}
$$


For $X \in \operatorname{Der}_{\mathcal{R}}(\mathcal{A})_{\mathcal{F}}$ we define a $\mathbb{k}$-linear map $X^{\mathcal{F}}: \mathcal{A} \rightarrow \mathcal{A}$ by

$$
X^{\mathcal{F}}(a)=\left(\mathcal{F}_{1}^{-1} \triangleright X\right)\left(\mathcal{F}_{2}^{-1} \triangleright a\right) \text { for all } a \in \mathcal{A} .
$$

This declares an isomorphism $\left(\mathfrak{X}_{\mathcal{R}}^{1}(\mathcal{A})\right)_{\mathcal{F}} \ni X \mapsto X^{\mathcal{F}} \in \mathfrak{X}_{\mathcal{R}_{\mathcal{F}}}^{1}\left(\mathcal{A}_{\mathcal{F}}\right)$ of $H_{\mathcal{F}}$-equivariant braided

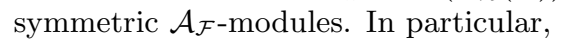

$$
\xi \triangleright X^{\mathcal{F}}=(\xi \triangleright X)^{\mathcal{F}}, a \cdot \cdot_{\mathcal{R}_{\mathcal{F}}} X^{\mathcal{F}}=(a \cdot \mathcal{F} X)^{\mathcal{F}}, X^{\mathcal{F}} \cdot{ }_{\mathcal{R}_{\mathcal{F}}} a=\left(X \cdot_{\mathcal{F}} a\right)^{\mathcal{F}}
$$

for all $\xi \in H, a \in \mathcal{A}$ and $X \in \mathfrak{X}_{\mathcal{R}}^{1}(\mathcal{A})_{\mathcal{F}}$, where we denoted the $\mathcal{A}_{\mathcal{F}}$-module actions on $\mathfrak{X}_{\mathcal{R}_{\mathcal{F}}}^{1}\left(\mathcal{A}_{\mathcal{F}}\right)$ by $\cdot_{\mathcal{F}}$. We define the twisted wedge product

$$
\wedge_{\mathcal{F}}=\operatorname{Drin}_{\mathcal{F}}\left(\wedge_{\mathcal{R}}\right) \circ \varphi_{\mathfrak{X}_{\mathcal{R}}^{1}(\mathcal{A}), \mathfrak{x}_{\mathcal{R}}^{1}(\mathcal{A})}: \mathfrak{X}_{\mathcal{R}}^{1}(\mathcal{A})_{\mathcal{F}} \otimes_{\mathcal{A}_{\mathcal{F}}} \mathfrak{X}_{\mathcal{R}}^{1}(\mathcal{A})_{\mathcal{F}} \rightarrow \mathfrak{X}_{\mathcal{R}}^{2}(\mathcal{A})_{\mathcal{F}}
$$

and extend the isomorphism (3.43) to higher wedge powers as a homomorphism of the twisted wedge product, i.e.

$$
\left(X \wedge_{\mathcal{F}} Y\right)^{\mathcal{F}}=X^{\mathcal{F}} \wedge_{\mathcal{R}_{\mathcal{F}}} Y^{\mathcal{F}}
$$

for all $X, Y \in \mathfrak{X}_{\mathcal{R}}^{\bullet}(\mathcal{A})_{\mathcal{F}}$, where $\wedge_{\mathcal{F}}=\operatorname{Drin}_{\mathcal{F}}\left(\wedge_{\mathcal{R}}\right) \circ \varphi_{\mathfrak{X}_{\mathcal{R}}(\mathcal{A}), \mathfrak{X}_{\mathcal{R}^{\prime}}(\mathcal{A})}$. Inductively this leads to an isomorphism $\mathfrak{X}_{\mathcal{R}}^{\bullet}(\mathcal{A})_{\mathcal{F}} \rightarrow \mathfrak{X}_{\mathcal{R}_{\mathcal{F}}}^{\bullet}\left(\mathcal{A}_{\mathcal{F}}\right)$ of $H_{\mathcal{F}}$-equivariant braided symmetric $\mathcal{A}_{\mathcal{F}}$-bimodules. Also the twisted Schouten-Nijenhuis bracket

$$
\llbracket \cdot, \cdot \rrbracket_{\mathcal{F}}: \operatorname{Drin}_{\mathcal{F}}\left(\llbracket \cdot, \cdot \rrbracket_{\mathcal{R}}\right) \circ \varphi_{\mathfrak{X}_{\mathcal{R}}(\mathcal{A}), \mathfrak{x}_{\mathcal{R}}^{\bullet}(\mathcal{A})}: \mathfrak{X}_{\mathcal{R}}^{\bullet}(\mathcal{A})_{\mathcal{F}} \otimes_{\mathcal{A}_{\mathcal{F}}} \mathfrak{X}_{\mathcal{R}}^{\bullet}(\mathcal{A})_{\mathcal{F}} \rightarrow \mathfrak{X}_{\mathcal{R}}^{\bullet}(\mathcal{A})_{\mathcal{F}}
$$

can be defined. On elements $X, Y \in \mathfrak{X}_{\mathcal{R}}^{\bullet}(\mathcal{A})_{\mathcal{F}}$ the twisted operations read

$$
X \wedge_{\mathcal{F}} Y=\left(\mathcal{F}_{1}^{-1} \triangleright X\right) \wedge_{\mathcal{R}}\left(\mathcal{F}_{2}^{-1} \triangleright Y\right) \text { and } \llbracket X, Y \rrbracket_{\mathcal{F}}=\llbracket \mathcal{F}_{1}^{-1} \triangleright X, \mathcal{F}_{2}^{-1} \triangleright Y \rrbracket_{\mathcal{R}},
$$

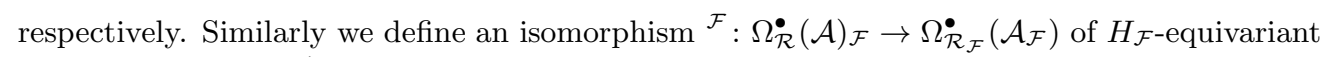
braided symmetric $\mathcal{A}_{\mathcal{F}}$-bimodules, the twisted Lie derivative and twisted insertion

$$
\begin{gathered}
\mathscr{L}^{\mathcal{F}}: \mathfrak{X}_{\mathcal{R}}^{\bullet}(\mathcal{A})_{\mathcal{F}} \otimes_{\mathcal{F}} \Omega_{\mathcal{R}}^{\bullet}(\mathcal{A})_{\mathcal{F}} \rightarrow \Omega_{\mathcal{R}}^{\bullet}(\mathcal{A})_{\mathcal{F}}, \\
\mathrm{i}^{\mathcal{F}}: \mathfrak{X}_{\mathcal{R}}^{\bullet}(\mathcal{A})_{\mathcal{F}} \otimes_{\mathcal{F}} \Omega_{\mathcal{R}}^{\bullet}(\mathcal{A})_{\mathcal{F}} \rightarrow \Omega_{\mathcal{R}}^{\bullet}(\mathcal{A})_{\mathcal{F}},
\end{gathered}
$$

while the de Rham differential becomes d: $\Omega_{\mathcal{R}}^{\bullet}(\mathcal{A})_{\mathcal{F}} \rightarrow \Omega_{\mathcal{R}}^{\bullet+1}(\mathcal{A})_{\mathcal{F}}$ after utilizing the Drinfel'd functor (see 41] for more details). On elements $X \in \mathfrak{X}_{\mathcal{R}}^{\bullet}(\mathcal{A})_{\mathcal{F}}$ and $\omega \in \Omega_{\mathcal{R}}^{\bullet}(\mathcal{A})_{\mathcal{F}}$ we obtain

$$
\mathscr{L}_{X}^{\mathcal{F}} \omega=\mathscr{L}_{\mathcal{F}_{1}^{-1} \triangleright X}^{\mathcal{R}}\left(\mathcal{F}_{2}^{-1} \triangleright \omega\right) \text { and } \mathrm{i}_{X}^{\mathcal{F}} \omega=\mathrm{i}_{\mathcal{F}_{1}^{-1} \triangleright X}^{\mathcal{R}}\left(\mathcal{F}_{2}^{-1} \triangleright \omega\right),
$$

while the de Rham differential remains undeformed. We refer to

$$
\left(\Omega_{\mathcal{R}}^{\bullet}(\mathcal{A})_{\mathcal{F}}, \wedge_{\mathcal{F}}, \mathscr{L}^{\mathcal{F}}, \mathrm{i}^{\mathcal{F}}, \mathrm{d}\right) \text { and }\left(\mathfrak{X}_{\mathcal{R}}^{\bullet}(\mathcal{A})_{\mathcal{F}}, \wedge_{\mathcal{F}}, \llbracket \cdot, \cdot \rrbracket_{\mathcal{F}}\right)
$$

as the twisted Cartan calculus with respect to $\mathcal{F}$ and $\mathcal{R}$.

Proposition 3.7. This assignment

$$
\mathcal{F}:\left(\mathfrak{X}_{\mathcal{R}}^{\bullet}(\mathcal{A})_{\mathcal{F}}, \wedge_{\mathcal{F}}, \llbracket \cdot, \cdot \rrbracket_{\mathcal{F}}\right) \rightarrow\left(\mathfrak{X}_{\mathcal{R}_{\mathcal{F}}}^{\bullet}\left(\mathcal{A}_{\mathcal{F}}\right), \wedge_{\mathcal{R}_{\mathcal{F}}}, \llbracket \cdot, \cdot \rrbracket_{\mathcal{R}_{\mathcal{F}}}\right),
$$

defined by eq. (3.43) and eq. (3.46), is an isomorphism of braided Gerstenhaber algebras and the twisted Cartan calculus with respect to $\mathcal{R}$ and $\mathcal{F}$ is isomorphic to the braided Cartan calculus on $\mathcal{A}_{\mathcal{F}}$ with respect to $\mathcal{R}_{\mathcal{F}}$ via the isomorphism ${ }^{\mathcal{F}}$. In particular

$$
\left(\llbracket X, Y \rrbracket_{\mathcal{F}}\right)^{\mathcal{F}}=\llbracket X^{\mathcal{F}}, Y^{\mathcal{F}} \rrbracket_{\mathcal{R}_{\mathcal{F}}},\left(\mathscr{L}_{X}^{\mathcal{F}} \omega\right)^{\mathcal{F}}=\mathscr{L}_{X^{\mathcal{F}}}^{\mathcal{R}_{\mathcal{F}}} \omega^{\mathcal{F}},\left(\mathrm{i}_{X}^{\mathcal{F}} \omega\right)^{\mathcal{F}}=\mathrm{i}_{X^{\mathcal{F}}}^{\mathcal{R}_{\mathcal{F}}} \omega^{\mathcal{F}},(\mathrm{d} \omega)^{\mathcal{F}}=\mathrm{d} \omega^{\mathcal{F}}
$$

for all $X, Y \in \mathfrak{X}_{\mathcal{R}}^{\bullet}(\mathcal{A})_{\mathcal{F}}$ and $\omega \in \Omega_{\mathcal{R}}^{\bullet}(\mathcal{A})_{\mathcal{F}}$.

Proof. By the inverse 2-cocycle property the twisted concatenation of $X, Y \in \operatorname{Der}(\mathcal{A})_{\mathcal{F}}$ equals

$$
\left(X \cdot_{\mathcal{F}} Y\right)^{\mathcal{F}}(a)=\left(\left(\mathcal{F}_{1(1)}^{-1} \mathcal{F}_{1}^{\prime-1}\right) \triangleright X\right)\left(\left(\mathcal{F}_{1(2)}^{-1} \mathcal{F}_{2}^{\prime-1}\right) \triangleright Y\right)\left(\mathcal{F}_{2}^{-1} \triangleright a\right)=\left(X^{\mathcal{F}} \cdot_{\mathcal{R}_{\mathcal{F}}} Y^{\mathcal{F}}\right)(a)
$$

for all $a \in \mathcal{A}$, where $\cdot_{\mathcal{R}_{\mathcal{F}}}$ denotes the concatenation of endomorphisms of $\mathcal{A}_{\mathcal{F}}$. Then

$$
\begin{aligned}
\left([X, Y]_{\mathcal{F}}\right)^{\mathcal{F}} & =\left(\left[\mathcal{F}_{1}^{-1} \triangleright X, \mathcal{F}_{2}^{-1} \triangleright Y\right]_{\mathcal{R}}\right)^{\mathcal{F}} \\
& =\left(\left(\mathcal{F}_{1}^{-1} \triangleright X\right) \cdot \mathcal{R}_{\mathcal{R}}\left(\mathcal{F}_{2}^{-1} \triangleright Y\right)\right)^{\mathcal{F}}-\left(\left(\left(\mathcal{R}_{1}^{-1} \mathcal{F}_{2}^{-1}\right) \triangleright Y\right) \cdot \mathcal{R}_{\mathcal{R}}\left(\left(\mathcal{R}_{2}^{-1} \mathcal{F}_{1}^{-1}\right) \triangleright X\right)\right)^{\mathcal{F}} \\
& =\left(X \cdot_{\mathcal{F}} Y\right)^{\mathcal{F}}-\left(\left(\mathcal{R}_{\mathcal{F} 1}^{-1} \triangleright Y\right) \cdot \mathcal{F}\left(\mathcal{R}_{\mathcal{F} 2}^{-1} \triangleright X\right)\right)^{\mathcal{F}} \\
& =X^{\mathcal{F}} \cdot_{\mathcal{R}_{\mathcal{F}}} Y^{\mathcal{F}}-\left(\mathcal{R}_{\mathcal{F} 1}^{-1} \triangleright Y^{\mathcal{F}}\right) \cdot_{\mathcal{R}_{\mathcal{F}}}\left(\mathcal{R}_{\mathcal{F} 2}^{-1} \triangleright X^{\mathcal{F}}\right) \\
& =\left[X^{\mathcal{F}}, Y^{\mathcal{F}}\right]_{\mathcal{R}_{\mathcal{F}}}
\end{aligned}
$$

where we also employed (3.44). Using formula (3.21) our previous computations together with eq.(3.46) imply $\left(\llbracket X, Y \rrbracket_{\mathcal{F}}\right)^{\mathcal{F}}=\llbracket X^{\mathcal{F}}, Y^{\mathcal{F}} \rrbracket_{\mathcal{R}_{\mathcal{F}}}$ for all $X, Y \in \mathfrak{X}_{\mathcal{R}}^{\bullet}(\mathcal{A})_{\mathcal{F}}$. The other equations follow similarly. We refer to [41] for a full proof. 
In other words, the above proposition shows that the twisted Cartan calculus is gauge equivalent to the untwisted Cartan calculus. Since the construction of the braided Cartan calculus is determined by the triangular structure and the twisted Cartan calculus is braided with respect to the twisted triangular structure our construction respects the gauge equivalence. In this light twist deformations seem trivial. On the other hand, there are situations where it is worth to distinguish the braided Cartan calculus and its twist deformations. Imagine for example a commutative left $H$-module algebra $\mathcal{A}$ for a cocommutative Hopf algebra $H$. For a nontrivial twist $\mathcal{F}$ on $H$ the twisted Cartan calculus is noncommutative while the untwisted one is commutative. In this sense one might consider the twisted Cartan calculus as a quantization of the untwisted one even if both are gauge equivalent. This might be interpreted as a quantization which is in 1-1-correspondence to its classical counterpart.

\subsection{Equivariant Covariant Derivatives and Metrics}

Having the braided Cartan calculus at hand we wonder if other concepts of differential geometry generalize to this setting. Focusing on the algebraic properties of covariant derivatives, namely function linearity in the first argument and a Leibniz rule in the second argument, we introduce equivariant covariant derivatives on equivariant braided symmetric bimodules. Note that there are several notions of covariant derivatives on noncommutative algebras (see e.g. 1, 2, 4, 5, 6, 9, 18, 25, 29, 35). In particular one has to distinguish between left and right covariant derivatives. In the spirit of these notes we demand the covariant derivative to be equivariant in addition, for which the definitions of left and right covariant derivatives coincide. Curvature and Torsion of equivariant covariant derivatives are discussed and we extend an equivariant covariant derivative on the algebra to braided multivector fields and differential forms. We furthermore give a generalization of metrics to the braided commutative setting and prove that there exists a unique equivariant Levi-Civita covariant derivative for every non-degenerate equivariant metric. Fix in the following a triangular Hopf algebra $(H, \mathcal{R})$ and a braided commutative left $H$-module algebra $\mathcal{A}$.

Definition 3.8 (Equivariant covariant derivative). Consider an $H$-equivariant braided symmetric $\mathcal{A}$-bimodule $\mathcal{M}$. An $H$-equivariant map $\nabla^{\mathcal{R}}: \mathfrak{X}_{\mathcal{R}}^{1}(\mathcal{A}) \otimes \mathcal{M} \rightarrow \mathcal{M}$ is said to be an equivariant covariant derivative on $\mathcal{M}$ with respect to $\mathcal{R}$, if for all $a \in \mathcal{A}, X \in \mathfrak{X}_{\mathcal{R}}^{1}(\mathcal{A})$ and $s \in \mathcal{M}$ one has

and

$$
\nabla_{a \cdot X}^{\mathcal{R}} s=a \cdot\left(\nabla_{X}^{\mathcal{R}} s\right)
$$

$$
\nabla_{X}^{\mathcal{R}}(a \cdot s)=\left(\mathscr{L}_{X}^{\mathcal{R}} a\right) \cdot s+\left(\mathcal{R}_{1}^{-1} \triangleright a\right) \cdot\left(\nabla_{\mathcal{R}_{2}^{-1} \triangleright X}^{\mathcal{R}} s\right) .
$$

Note that $H$-equivariance of a $\mathbb{k}$-linear map $\nabla^{\mathcal{R}}: \mathfrak{X}_{\mathcal{R}}^{1}(\mathcal{A}) \otimes \mathcal{M} \rightarrow \mathcal{M}$ reads $\xi \triangleright\left(\nabla_{X}^{\mathcal{R}} s\right)=$ $\nabla_{\xi_{(1)} \triangleright X X}^{\mathcal{R}}\left(\xi_{(2)} \triangleright s\right)$ for all $\xi \in H, X \in \mathfrak{X}_{\mathcal{R}}^{1}(\mathcal{A})$ and $s \in \mathcal{M}$. The curvature of an equivariant covariant derivative $\nabla^{\mathcal{R}}$ on $\mathcal{M}$ is defined by

$$
R^{\nabla^{\mathcal{R}}}(X, Y)=\nabla_{X}^{\mathcal{R}} \nabla_{Y}^{\mathcal{R}}-\nabla_{\mathcal{R}_{1}^{-1} \triangleright Y}^{\mathcal{R}} \nabla_{\mathcal{R}_{2}^{-1} \triangleright X}^{\mathcal{R}}-\nabla_{[X, Y]_{\mathcal{R}}}^{\mathcal{R}}
$$

for $X, Y \in \mathfrak{X}_{\mathcal{R}}^{1}(\mathcal{A})$. If $\mathcal{M}=\mathfrak{X}_{\mathcal{R}}^{1}(\mathcal{A})$ we can further define the torsion of $\nabla^{\mathcal{R}}$ by

$$
\operatorname{Tor}^{\nabla^{\mathcal{R}}}(X, Y)=\nabla_{X}^{\mathcal{R}} Y-\nabla_{\mathcal{R}_{1}^{-1} \triangleright Y}^{\mathcal{R}}\left(\mathcal{R}_{2}^{-1} \triangleright X\right)-[X, Y]_{\mathcal{R}},
$$

for all $X, Y \in \mathfrak{X}_{\mathcal{R}}^{1}(\mathcal{A})$. An equivariant covariant derivative $\nabla^{\mathcal{R}}$ is flat if $R^{\nabla^{\mathcal{R}}}=0$ and torsionfree if $\operatorname{Tor}^{\nabla^{\mathcal{R}}}=0$. While eq. (3.54) and eq.(3.55) usually only refer to a left covariant derivative we prove in the following lemma (c.f. [41) that in the equivariant setup the notions of left and right covariant derivatives are equivalent.

Lemma 3.9. Let $\nabla^{\mathcal{R}}$ be a covariant derivative on an $H$-equivariant braided symmetric $\mathcal{A}$ bimodule $\mathcal{M}$. Then for all $a \in \mathcal{A}, X \in \mathfrak{X}_{\mathcal{R}}^{1}(\mathcal{A})$ and $s \in \mathcal{M}$,

$$
\nabla_{X \cdot a}^{\mathcal{R}} s=\left(\nabla_{X}^{\mathcal{R}}\left(\mathcal{R}_{1}^{-1} \triangleright s\right)\right) \cdot\left(\mathcal{R}_{2}^{-1} \triangleright a\right)
$$

and

$$
\nabla_{X}^{\mathcal{R}}(s \cdot a)=\left(\nabla_{X}^{\mathcal{R}} s\right) \cdot a+\left(\mathcal{R}_{1}^{-1} \triangleright s\right) \cdot\left(\mathscr{L}_{\mathcal{R}_{2}^{-1} \triangleright X}^{\mathcal{R}} a\right)
$$

hold. On the other hand, every $H$-equivariant map $\nabla^{\mathcal{R}}: \mathfrak{X}_{\mathcal{R}}^{1}(\mathcal{A}) \otimes \mathcal{M} \rightarrow \mathcal{M}$ satisfying eq. (3.58) and eq. (3.59) is an equivariant covariant derivative on $\mathcal{M}$.

There are natural extensions of an equivariant covariant derivative $\nabla^{\mathcal{R}}: \mathfrak{X}_{\mathcal{R}}^{1}(\mathcal{A}) \otimes \mathfrak{X}_{\mathcal{R}}^{1}(\mathcal{A}) \rightarrow$ $\mathfrak{X}_{\mathcal{R}}^{1}(\mathcal{A})$ to braided multivector fields and differential forms in analogy to differential geometry. We define the braided dual pairing $\langle\cdot, \cdot\rangle_{\mathcal{R}}: \Omega_{\mathcal{R}}^{1}(\mathcal{A}) \otimes \mathfrak{X}_{\mathcal{R}}^{1}(\mathcal{R}) \rightarrow \mathcal{A}$ by $\langle\omega, X\rangle_{\mathcal{R}}=\omega(X)$ for all $\omega \in \Omega_{\mathcal{R}}^{1}(\mathcal{A})$ and $X \in \mathfrak{X}_{\mathcal{R}}^{1}(\mathcal{A})$. It is $H$-equivariant, left $\mathcal{A}$-linear in the first and right $\mathcal{A}$-linear in the second argument. 
Proposition 3.10. An equivariant covariant derivative $\nabla^{\mathcal{R}}$ on $\mathfrak{X}_{\mathcal{R}}^{1}(\mathcal{A})$ induces an equivariant covariant derivative $\tilde{\nabla}^{\mathcal{R}}$ on $\Omega_{\mathcal{R}}^{1}(\mathcal{A})$ via

$$
\left\langle\tilde{\nabla}_{X}^{\mathcal{R}} \omega, Y\right\rangle_{\mathcal{R}}=\mathscr{L}_{X}^{\mathcal{R}}\langle\omega, Y\rangle_{\mathcal{R}}-\left\langle\mathcal{R}_{1}^{-1} \triangleright \omega, \nabla_{\mathcal{R}_{2}^{-1} \triangleright X}^{\mathcal{R}} Y\right\rangle_{\mathcal{R}}
$$

for all $X, Y \in \mathfrak{X}_{\mathcal{R}}^{1}(\mathcal{A})$ and $\omega \in \Omega_{\mathcal{R}}^{1}(\mathcal{A})$. Moreover, $\nabla^{\mathcal{R}}$ and $\tilde{\nabla}^{\mathcal{R}}$ can be extended as braided derivations to equivariant covariant derivatives on $\mathfrak{X}_{\mathcal{R}}^{\bullet}(\mathcal{A})$ and $\Omega_{\mathcal{R}}^{\bullet}(\mathcal{A})$, respectively.

Proof. Let $X, Y \in \mathfrak{X}_{\mathcal{R}}^{1}(\mathcal{A}), \omega \in \Omega_{\mathcal{R}}^{1}(\mathcal{A})$ and $a \in \mathcal{A}$. Then $\tilde{\nabla}_{X}^{\mathcal{R}} \omega \in \Omega_{\mathcal{R}}^{1}(\mathcal{A})$ is well-defined, since

$$
\begin{aligned}
\left\langle\tilde{\nabla}_{X}^{\mathcal{R}} \omega, Y \cdot a\right\rangle_{\mathcal{R}}= & \mathscr{L}_{X}^{\mathcal{R}}\langle\omega, Y \cdot a\rangle_{\mathcal{R}}-\left\langle\mathcal{R}_{1}^{-1} \triangleright \omega, \nabla_{\mathcal{R}_{2}^{-1} \triangleright X}^{\mathcal{R}}(Y \cdot a)\right\rangle_{\mathcal{R}} \\
= & \left(\mathscr{L}_{X}^{\mathcal{R}}\langle\omega, Y\rangle_{\mathcal{R}}\right) \cdot a+\left(\mathcal{R}_{1}^{-1} \triangleright\langle\omega, Y\rangle_{\mathcal{R}}\right) \cdot \mathscr{L}_{\mathcal{R}_{2}^{-1} \triangleright X}^{\mathcal{R}} a \\
& -\left\langle\mathcal{R}_{1}^{-1} \triangleright \omega,\left(\nabla_{\mathcal{R}_{2}^{-1} \triangleright X}^{\mathcal{R}} Y\right) \cdot a+\left(\mathcal{R}_{1}^{\prime-1} \triangleright Y\right) \cdot \mathscr{L}_{\mathcal{R}_{2}^{\prime}-1}^{\mathcal{R}} \mathcal{R}_{2}^{-1} \triangleright X\right. \\
& a\rangle_{\mathcal{R}} \\
= & \left\langle\tilde{\nabla}_{X}^{\mathcal{R}} \omega, Y\right\rangle_{\mathcal{R}} \cdot a .
\end{aligned}
$$

Similarly one proves that $\tilde{\nabla}^{\mathcal{R}}$ is left $\mathcal{A}$-linear in the first argument and satisfies the braided Leibniz rule in the second argument. For another $\eta \in \Omega_{\mathcal{R}}^{1}(\mathcal{A})$ one verifies that

$$
\tilde{\nabla}_{X}^{\mathcal{R}}\left(\omega \wedge_{\mathcal{R}} \eta\right)=\tilde{\nabla}_{X}^{\mathcal{R}} \omega \wedge_{\mathcal{R}} \eta+\left(\mathcal{R}_{1}^{-1} \triangleright \omega\right) \wedge_{\mathcal{R}} \tilde{\nabla}_{\mathcal{R}_{2}^{-1} \triangleright X}^{\mathcal{R}} \eta
$$

defines an equivariant covariant derivative on $\Omega_{\mathcal{R}}^{2}(\mathcal{A})$ and inductively $\tilde{\nabla}^{\mathcal{R}}$ extends as a braided derivation of $\wedge_{\mathcal{R}}$ to $\Omega_{\mathcal{R}}^{\bullet}(\mathcal{A})$. The extension of $\nabla^{\mathcal{R}}$ to braided multivector fields is entirely similar.

Let $\nabla^{\mathcal{R}}: \mathfrak{X}_{\mathcal{R}}^{1}(\mathcal{A}) \otimes \mathcal{M} \rightarrow \mathcal{M}$ be an equivariant covariant derivative with respect to $\mathcal{R}$ on an $H$-equivariant braided symmetric $\mathcal{A}$-bimodule $\mathcal{M}$. For any twist $\mathcal{F}$ on $H$ we define the twisted equivariant covariant derivative

$$
\nabla^{\mathcal{F}}=\operatorname{Drin}_{\mathcal{F}}\left(\nabla^{\mathcal{R}}\right) \circ \varphi_{\mathfrak{X}_{\mathcal{R}}^{1}(\mathcal{A}), \mathcal{M}}: \mathfrak{X}_{\mathcal{R}}^{1}(\mathcal{A})_{\mathcal{F}} \otimes_{\mathcal{F}} \mathcal{M}_{\mathcal{F}} \rightarrow \mathcal{M}_{\mathcal{F}}
$$

which reads

$$
\nabla_{X}^{\mathcal{F}} s=\nabla_{\mathcal{F}_{1}^{-1} \triangleright X}^{\mathcal{R}}\left(\mathcal{F}_{2}^{-1} \triangleright s\right) .
$$

on elements $X \in \mathfrak{X}_{\mathcal{R}}^{1}(\mathcal{A})_{\mathcal{F}}$ and $s \in \mathcal{M}_{\mathcal{F}}$.

Proposition 3.11. The twisted equivariant covariant derivative is an equivariant covariant derivative with respect to the twisted triangular structure, where we identify $\mathfrak{X}_{\mathcal{R}}^{1}(\mathcal{A})_{\mathcal{F}}$ with $\mathfrak{X}_{\mathcal{R}_{\mathcal{F}}}^{1}\left(\mathcal{A}_{\mathcal{F}}\right)$ according to Proposition 3.7 .

Proof. Let $\xi \in H, a \in \mathcal{A}, X \in \mathfrak{X}_{\mathcal{R}}^{1}(\mathcal{A})_{\mathcal{F}}$ and $s \in \mathcal{M}_{\mathcal{F}}$. Then

$$
\xi \triangleright\left(\nabla_{X}^{\mathcal{F}} s\right)=\nabla_{\left(\xi_{(1)} \mathcal{F}_{1}^{-1}\right) \triangleright X}^{\mathcal{R}}\left(\left(\xi_{(2)} \mathcal{F}_{2}^{-1}\right) \triangleright s\right)=\nabla_{\xi_{(1)}^{\mathcal{F}} \triangleright X}^{\mathcal{F}}\left(\xi_{\widehat{(2)}} \triangleright s\right)
$$

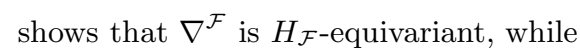

$$
\begin{aligned}
\nabla_{a \cdot \mathcal{F} X}^{\mathcal{F}} s & =\left(\left(\mathcal{F}_{1(1)}^{-1} \mathcal{F}_{1}^{\prime}-1\right) \triangleright a\right) \cdot\left(\nabla_{\left(\mathcal{F}_{1(2)}^{-1} \mathcal{F}_{2}^{\prime-1}\right) \triangleright X}^{\mathcal{R}}\left(\mathcal{F}_{2}^{-1} \triangleright s\right)\right) \\
& =\left(\mathcal{F}_{1}^{-1} \triangleright a\right) \cdot\left(\nabla_{\left(\mathcal{F}_{2(1)}^{-1} \mathcal{F}_{1}^{\prime-1}\right) \triangleright X}^{\mathcal{R}}\left(\left(\mathcal{F}_{2(2)}^{-1} \mathcal{F}_{2}^{\prime}-1\right) \triangleright s\right)\right) \\
& =\left(\mathcal{F}_{1}^{-1} \triangleright a\right) \cdot\left(\mathcal{F}_{2}^{-1} \triangleright\left(\nabla_{\mathcal{F}_{1}^{\prime-1} \triangleright X}^{\mathcal{R}}\left(\mathcal{F}_{2}^{\prime-1} \triangleright s\right)\right)\right) \\
& =a \cdot \mathcal{F}\left(\nabla_{X}^{\mathcal{F}} s\right)
\end{aligned}
$$

and

$$
\begin{aligned}
\nabla_{X}^{\mathcal{F}}(a \cdot \mathcal{F} s)= & \nabla_{\mathcal{F}_{1}^{-1} \triangleright X}^{\mathcal{R}}\left(\left(\left(\mathcal{F}_{2(1)}^{-1} \mathcal{F}_{1}^{\prime}-1\right) \triangleright a\right) \cdot\left(\left(\mathcal{F}_{2(1)}^{-1} \mathcal{F}_{1}^{\prime}-1\right) \triangleright a\right)\right) \\
= & \left(\mathscr{L}_{\mathcal{F}_{1}^{\mathcal{R}} \triangleright X}^{-1}\left(\left(\mathcal{F}_{2(1)}^{-1} \mathcal{F}_{1}^{\prime-1}\right) \triangleright a\right)\right) \cdot\left(\left(\mathcal{F}_{2(2)}^{-1} \mathcal{F}_{2}^{\prime}-1\right) \triangleright s\right) \\
& +\left(\left(\mathcal{R}_{1}^{-1} \mathcal{F}_{2(1)}^{-1} \mathcal{F}_{1}^{\prime-1}\right) \triangleright a\right) \cdot\left(\nabla_{\left(\mathcal{R}_{2}^{-1} \mathcal{F}_{1}^{-1}\right) \triangleright X}^{\mathcal{R}}\left(\left(\mathcal{F}_{2(2)}^{-1} \mathcal{F}_{2}^{\prime-1}\right) \triangleright s\right)\right) \\
= & \left(\mathcal{F}_{1}^{-1} \triangleright\left(\mathscr{L}_{\mathcal{F}_{1}^{\prime}-1}^{\mathcal{R}}\left(\mathcal{F}_{2}^{\prime-1} \triangleright a\right)\right)\right) \cdot\left(\mathcal{F}_{2}^{-1} \triangleright s\right) \\
& +\left(\left(\mathcal{F}_{2(1)}^{-1} \mathcal{R}_{1}^{-1} \mathcal{F}_{2}^{\prime}{ }^{-1}\right) \triangleright a\right) \cdot\left(\nabla_{\left(\mathcal{F}_{2(2)}^{-1} \mathcal{R}_{2}^{-1} \mathcal{F}_{1}^{\prime-1}\right) \triangleright X}^{\mathcal{R}}\left(\mathcal{F}_{2}^{-1} \triangleright s\right)\right) \\
= & \left(\mathscr{L}_{X}^{\mathcal{F}} a\right) \cdot \mathcal{F} s+\left(\left(\mathcal{F}_{1(1)}^{-1} \mathcal{F}_{1}^{-1} \mathcal{R}_{\mathcal{F} 1}^{-1}\right) \triangleright a\right) \cdot\left(\nabla_{\left(\mathcal{F}_{1(2)}^{-1} \mathcal{F}_{2}^{-1} \mathcal{R}_{\mathcal{F} 2}^{-1}\right) \triangleright X}^{\mathcal{R}}\left(\mathcal{F}_{2}^{-1} \triangleright s\right)\right) \\
= & \left(\mathscr{L}_{X}^{\mathcal{F}} a\right) \cdot \mathcal{F} s+\left(\mathcal{R}_{\mathcal{F} 1}^{-1} \triangleright a\right) \cdot \mathcal{F}\left(\nabla_{\mathcal{R}_{\mathcal{F} 2} \triangleright X}^{\mathcal{F}} s\right)
\end{aligned}
$$

are the correct linearity properties, proving that $\nabla^{\mathcal{F}}$ is an equivariant covariant derivative with respect to $\mathcal{R}_{\mathcal{F}}$. 
In Riemannian geometry covariant derivatives are always considered together with a Riemannian metric. We want to generalize them to braided commutative algebras: a $\mathbb{k}$-linear map $\mathbf{g}: \mathfrak{X}_{\mathcal{R}}^{1}(\mathcal{A}) \otimes_{\mathcal{A}} \mathfrak{X}_{\mathcal{R}}^{1}(\mathcal{A}) \rightarrow \mathcal{A}$, which is left $\mathcal{A}$-linear in the first argument and $H$-equivariant, is said to be an equivariant metric if it is braided symmetric, i.e. if $\mathbf{g}(Y, X)=\mathbf{g}\left(\mathcal{R}_{1}^{-1} \triangleright X, \mathcal{R}_{2}^{-1} \triangleright Y\right)$ for all $X, Y \in \mathfrak{X}_{\mathcal{R}}^{1}(\mathcal{A})$. It follows that $\mathbf{g}$ is braided right $\mathcal{A}$-linear in the first argument as well as right $\mathcal{A}$-linear and braided left $\mathcal{A}$-linear in the second argument. An equivariant metric is said to be non-degenerate if $\mathbf{g}(X, Y)=0$ for all $Y \in \mathfrak{X}_{\mathcal{R}}^{1}(\mathcal{A})$ implies $X=0$, it is said to be strongly non-degenerate if $\mathbf{g}(X, X) \neq 0$ for all $X \neq 0$ and it is said to be Riemannian if it is strongly non-degenerate and there is a partial order $\geq$ on $\mathcal{A}$ such that $\mathbf{g}(X, X) \geq 0$ for all $X \neq 0$ in addition. Note that strongly non-degeneracy implies non-degeneracy. An equivariant covariant derivative $\nabla^{\mathcal{R}}: \mathfrak{X}_{\mathcal{R}}^{1}(\mathcal{A}) \otimes \mathfrak{X}_{\mathcal{R}}^{1}(\mathcal{A}) \rightarrow \mathfrak{X}_{\mathcal{R}}^{1}(\mathcal{A})$ on $\mathcal{A}$ is said to be a metric equivariant covariant derivative with respect to an equivariant metric $\mathbf{g}$, if

$$
\mathscr{L}_{X}^{\mathcal{R}}(\mathbf{g}(Y, Z))=\mathbf{g}\left(\nabla_{X}^{\mathcal{R}} Y, Z\right)+\mathbf{g}\left(\mathcal{R}_{1}^{-1} \triangleright Y, \nabla_{\mathcal{R}_{2}^{-1} \triangleright X}^{\mathcal{R}} Z\right)
$$

holds for all $X, Y, Z \in \mathfrak{X}_{\mathcal{R}}^{1}(\mathcal{A})$. Note that equivariance $\xi \triangleright \mathbf{g}(X, Y)=\mathbf{g}\left(\xi_{(1)} \triangleright X, \xi_{(2)} \triangleright Y\right)$ for all $\xi \in H$ and $X, Y \in \mathfrak{X}_{\mathcal{R}}^{1}(\mathcal{A})$, of a metric is a quite strong requirement. Similar approaches which omit this condition are e.g. 2, 5, 25.

Lemma 3.12. Let $\mathbf{g}$ be a non-degenerate equivariant metric on $\mathcal{A}$. Then there is a unique torsion-free metric equivariant covariant derivative on $\mathcal{A}$.

Proof. Fix an equivariant metric g on $\mathcal{A}$. Any equivariant covariant derivative $\nabla^{\mathcal{R}}$ on $\mathcal{A}$, which is torsion free and metric with respect to $\mathbf{g}$, satisfies

$$
\begin{aligned}
2 \mathbf{g}\left(\nabla_{X}^{\mathcal{R}} Y, Z\right)= & X(\mathbf{g}(Y, Z))+\left(\mathcal{R}_{1(1)}^{-1} \triangleright Y\right)\left(\mathbf{g}\left(\mathcal{R}_{1(2)}^{-1} \triangleright Z, \mathcal{R}_{2}^{-1} \triangleright X\right)\right) \\
& -\left(\mathcal{R}_{1}^{-1} \triangleright Z\right)\left(\mathbf{g}\left(\mathcal{R}_{2(1)}^{-1} \triangleright X, \mathcal{R}_{2(2)}^{-1} \triangleright Y\right)\right) \\
& -\mathbf{g}\left(X,[Y, Z]_{\mathcal{R}}\right)+\mathbf{g}\left(\mathcal{R}_{1(1)}^{-1} \triangleright Y,\left[\mathcal{R}_{1(2)}^{-1} \triangleright Z, \mathcal{R}_{2}^{-1} \triangleright X\right]_{\mathcal{R}}\right) \\
& +\mathbf{g}\left(\mathcal{R}_{1}^{-1} \triangleright Z,\left[\mathcal{R}_{2(1)}^{-1} \triangleright X, \mathcal{R}_{2(2)}^{-1} \triangleright Y\right]_{\mathcal{R}}\right)
\end{aligned}
$$

for all $X, Y, Z \in \mathfrak{X}_{\mathcal{R}}^{1}(\mathcal{A})$. In particular, this shows the uniqueness of a torsion-free equivariant covariant derivative which is metric with respect to $\mathbf{g}$, if $\mathbf{g}$ is non-degenerate. It remains to prove that a $\mathbb{k}$-bilinear map $\nabla^{\mathcal{R}}$ determined by the above formula is a metric torsion-free equivariant covariant derivative. This follows by the (braided) linearity properties of $\mathbf{g}$ and the braided Leibniz rule. A full proof can be found in [41.

The unique torsion-free metric equivariant covariant derivative on $(\mathcal{A}, \mathbf{g})$ is said to be the equivariant Levi-Civita covariant derivative. We want to remark that Lemma 3.12 admits a generalization in the sense that for any value of the torsion there exists a unique metric equivariant covariant derivative. As a last observation of this section we prove that the twist deformation of an equivariant metric is an equivariant metric on the twisted algebra and the assignment LC: $\mathbf{g} \mapsto \nabla^{\mathrm{LC}}$, attributing to a non-degenerate equivariant metric its equivariant Levi-Civita covariant derivative, respects the Drinfel'd functor.

Corollary 3.13. Let $\mathbf{g}$ be an equivariant metric on $\mathcal{A}$. Then, the twisted equivariant metric $\mathbf{g}_{\mathcal{F}}$, which is defined by

$$
\mathbf{g}_{\mathcal{F}}(X, Y)=\mathbf{g}\left(\mathcal{F}_{1}^{-1} \triangleright X, \mathcal{F}_{2}^{-1} \triangleright Y\right)
$$

for all $X, Y \in \mathfrak{X}_{\mathcal{R}}^{1}(\mathcal{A})$, is an equivariant metric with respect to $\mathcal{R}_{\mathcal{F}}$ on $\mathcal{A}_{\mathcal{F}}$. Moreover, assuming that $\mathbf{g}$ and $\mathbf{g}_{\mathcal{F}}$ are non-degenerate, twisting the equivariant Levi-Civita covariant derivative with respect to $\mathbf{g}$ leads to the equivariant Levi-Civita covariant derivative with respect to $\mathbf{g}_{\mathcal{F}}$.

Proof. One immediately verifies that $\mathbf{g}_{\mathcal{F}}$ is an $H_{\mathcal{F}}$-equivariant left $\mathcal{A}_{\mathcal{F}}$-linear map $\mathfrak{X}_{\mathcal{R}}^{1}(\mathcal{A})_{\mathcal{F}} \otimes_{\mathcal{A}_{\mathcal{F}}}$ $\mathfrak{X}_{\mathcal{R}}^{1}(\mathcal{A})_{\mathcal{F}} \rightarrow \mathcal{A}_{\mathcal{F}}$ which is braided symmetric with respect to $\mathcal{R}_{\mathcal{F}}$. Via the identification $\mathfrak{X}_{\mathcal{R}}^{1}(\mathcal{A})_{\mathcal{F}} \cong \mathfrak{X}_{\mathcal{R}_{\mathcal{F}}}^{1}\left(\mathcal{A}_{\mathcal{F}}\right)$ of Proposition $3.7 \mathbf{g}_{\mathcal{F}}$ becomes an equivariant metric on $\mathcal{A}_{\mathcal{F}}$. Let $X, Y, Z \in \mathfrak{X}_{\mathcal{R}}^{1}(\mathcal{A})$ and denote the equivariant Levi-Civita covariant derivative of $\mathbf{g}$ by $\nabla^{\mathcal{R}}$. From eq.(3.64) it follows that the twisted equivariant covariant derivative $\nabla^{\mathcal{F}}$ satisfies

$$
\mathscr{L}_{X}^{\mathcal{F}}\left(\mathbf{g}_{\mathcal{F}}(Y, Z)\right)=\mathbf{g}_{\mathcal{F}}\left(\nabla_{X}^{\mathcal{F}} Y, Z\right)+\mathbf{g}_{\mathcal{F}}\left(\mathcal{R}_{\mathcal{F} 1}^{-1} \triangleright Y, \nabla_{\mathcal{R}_{\mathcal{F} 2}^{-1} \triangleright X}^{\mathcal{F}} Z\right) .
$$

Since $\operatorname{Tor}^{\nabla^{\mathcal{R}}}=0$ we obtain $\operatorname{Tor}^{\nabla^{\mathcal{F}}}=0$, proving that $\nabla^{\mathcal{F}}$ is the unique equivariant Levi-Civita covariant derivative corresponding to $\mathbf{g}_{\mathcal{F}}$ if the latter is non-degenerate. 


\section{Submanifolds in Braided Commutative Geometry}

In this section we show that the braided Cartan calculus is compatible with the concept of submanifold algebras if the triangular Hopf algebra respects the corresponding submanifold ideal. This can be understood as a construction of new examples of braided Cartan calculi from known ones. The projection to submanifold algebras respects Drinfel'd twist gauge equivalence classes, which is an interesting supplement to Proposition 3.7 The second subsection is devoted to the study of equivariant covariant derivatives on submanifold algebras. Depending on the choice of a strongly non-degenerate equivariant metric one is able to project equivariant covariant derivatives as well as curvature and torsion if the submanifold algebra obeys two mild axioms. Furthermore, the notion of twisted equivariant covariant derivative and metric are compatible with the projections. While the main Section 3 stands out with quite an amount of details, we are relatively short-spoken in the present section. The interested reader is relegated to 41 for a more circumstantial discussion. A different approach to Riemannian geometry on noncommutative submanifolds, based on the choice of a finite-dimensional Lie subalgebra $\mathfrak{g}$ of $\operatorname{Der}(\mathcal{A})$ and a vector space homomorphism $\mathfrak{g} \rightarrow \mathcal{M}$ into a right $\mathcal{A}$-module $\mathcal{M}$, is considered in [1. Yet another approach to noncommutative (fuzzy) submanifolds $S$ of $\mathbb{R}^{n}$, based on the imposition of an energy cutoff on a quantum particle in $\mathbb{R}^{n}$, subject to a confining potential with a very sharp minimum on $S$, has been recently proposed and applied to spheres in [24].

\subsection{Braided Cartan Calculi on Submanifolds}

In noncommutative geometry there is a well-known notion of submanifold ideal (c.f. [32]) generalizing the concept of closed embedded smooth submanifolds. We refer to [14] for a recent discussion of submanifold algebras. In braided commutative geometry the submanifold ideals have to be respected by the Hopf algebra action in order to inherit a braided symmetry on the quotient algebra. We continue by describing the braided Cartan calculus on the submanifold algebra in this situation. It is given by the projection of the braided Cartan calculus of the ambient algebra. Moreover, the Drinfel'd functor intertwines the submanifold algebra projections. The following discussion is also motivated by [22, 23 .

Fix a triangular Hopf algebra $(H, \mathcal{R})$ and a braided commutative left $H$-module algebra $\mathcal{A}$. For any algebra ideal $\mathcal{C} \subseteq \mathcal{A}$ the coset space $\mathcal{A} / \mathcal{C}$ becomes an algebra with unit and product induced from $\mathcal{A}$. The elements of $\mathcal{A} / \mathcal{C}$ are equivalence classes of elements in $\mathcal{A}$, where $a, b \in \mathcal{A}$ are identified if and only if there exists an element $c \in \mathcal{C}$ such that $a=b+c$. The corresponding surjective projection is denoted by $\operatorname{pr}: \mathcal{A} \ni a \mapsto a+\mathcal{C} \in \mathcal{A} / \mathcal{C}$. If the left $H$-module action respects $\mathcal{C}$, i.e. if $H \triangleright \mathcal{C} \subseteq \mathcal{C}$, the quotient $\mathcal{A} / \mathcal{C}$ is a braided commutative left $H$-module algebra with respect to $\mathcal{R}$ and the left $H$-action defined by $\xi \triangleright \operatorname{pr}(a)=\operatorname{pr}(\xi \triangleright a)$ for all $a \in \mathcal{A}$. Braided vector fields on the braided commutative algebra $\mathcal{A} / \mathcal{C}$ can be obtained as projections from a certain class of braided vector fields on $\mathcal{A}$. A braided derivation $X \in \operatorname{Der}_{\mathcal{R}}(\mathcal{A})$ is said to be tangent to $\mathcal{C}$ if $X(\mathcal{C}) \subseteq \mathcal{C}$. The $\mathbb{k}$-module of all braided derivations of $\mathcal{A}$ which are tangent to $\mathcal{C}$ is denoted by $\mathfrak{X}_{t}^{1}(\mathcal{A})$. It is a braided Lie subalgebra and an $H$-equivariant braided symmetric $\mathcal{A}$-sub-bimodule of $\mathfrak{X}_{\mathcal{R}}^{1}(\mathcal{A})$. Consider the $\mathbb{k}$-linear map

$$
\text { pr: } \mathfrak{X}_{t}^{1}(\mathcal{A}) \rightarrow \operatorname{Der}_{\mathcal{R}}(\mathcal{A} / \mathcal{C}),
$$

defined for any $X \in \mathfrak{X}_{t}^{1}(\mathcal{A})$ by $\operatorname{pr}(X)(\operatorname{pr}(a))=\operatorname{pr}(X(a))$ for all $a \in \mathcal{A}$. Note that there are left and right $\mathcal{A} / \mathcal{C}$-actions and an $H$-action on the image of (4.1) defined by

$$
\xi \triangleright \operatorname{pr}(X)=\operatorname{pr}(\xi \triangleright X), \operatorname{pr}(a) \cdot \operatorname{pr}(X)=\operatorname{pr}(a \cdot X), \operatorname{pr}(X) \cdot \operatorname{pr}(a)=\operatorname{pr}(X \cdot a)
$$

for all $\xi \in H, a \in \mathcal{A}$ and $X \in \mathfrak{X}_{t}^{1}(\mathcal{A})$. Those structure the image $\operatorname{pr}\left(\mathfrak{X}_{t}^{1}(\mathcal{A})\right) \subseteq \mathfrak{X}_{\mathcal{R}}^{1}(\mathcal{A})$ as an $H$-equivariant braided symmetric $\mathcal{A} / \mathcal{C}$-sub-bimodule and braided Lie subalgebra. On the other hand $\operatorname{pr}\left(\mathfrak{X}_{t}^{1}(\mathcal{A})\right)$ can be viewed as an $H$-equivariant braided symmetric $\mathcal{A}$-bimodule with $\mathcal{A}$-actions $a \cdot \operatorname{pr}(X)=\operatorname{pr}(a) \cdot \operatorname{pr}(X)$ and $\operatorname{pr}(X) \cdot a=\operatorname{pr}(X) \cdot \operatorname{pr}(a)$ for all $a \in \mathcal{A}$ and $X \in \mathfrak{X}_{t}^{1}(\mathcal{A})$. With respect to the latter structures (4.1) becomes a homomorphism of $H$-equivariant braided symmetric $\mathcal{A}$-bimodules and braided Lie algebras. It follows that the kernel $\mathfrak{X}_{0}^{1}(\mathcal{A})$ of (4.1) is an $H$-equivariant braided symmetric $\mathcal{A}$-sub-bimodule and a braided Lie ideal of $\mathfrak{X}_{t}^{1}(\mathcal{A})$.

Definition 4.1. An algebra ideal $\mathcal{C} \subseteq \mathcal{A}$ is said to be a submanifold ideal and the quotient $\mathcal{A} / \mathcal{C}$ is said to be a submanifold algebra if there is a short exact sequence

$$
0 \rightarrow \mathfrak{X}_{0}^{1}(\mathcal{A}) \rightarrow \mathfrak{X}_{t}^{1}(\mathcal{A}) \stackrel{\text { pr }}{\rightarrow} \operatorname{Der}_{\mathcal{R}}(\mathcal{A} / \mathcal{C}) \rightarrow 0
$$

of $H$-equivariant braided symmetric $\mathcal{A}$-bimodules and braided Lie algebras. 
Fix a submanifold ideal $\mathcal{C}$ of $\mathcal{A}$ in the following. The short exact sequence (4.3) extends to a short exact sequence

$$
0 \rightarrow \mathfrak{X}_{0}^{\bullet}(\mathcal{A}) \rightarrow \mathfrak{X}_{t}^{\bullet}(\mathcal{A}) \stackrel{\mathrm{pr}}{\longrightarrow} \mathfrak{X}_{\mathcal{R}}^{\bullet}(\mathcal{A} / \mathcal{C}) \rightarrow 0
$$

of braided Gerstenhaber algebras by defining inductively $\operatorname{pr}\left(X \wedge_{\mathcal{R}} Y\right)=(\operatorname{pr}(X)) \wedge_{\mathcal{R}}(\operatorname{pr}(Y))$ for all $X, Y \in \mathfrak{X}_{t}^{\bullet}(\mathcal{A})$, where $\mathfrak{X}_{0}^{\bullet}(\mathcal{A})$ and $\mathfrak{X}_{t}^{\bullet}(\mathcal{A})$ denote the braided exterior algebras of $\mathfrak{X}_{0}^{1}(\mathcal{A})$ and $\mathfrak{X}_{t}^{1}(\mathcal{A})$, respectively. In particular $\operatorname{pr}\left(\llbracket X, Y \rrbracket_{\mathcal{R}}\right)=\llbracket \operatorname{pr}(X), \operatorname{pr}(Y) \rrbracket_{\mathcal{R}}$ holds for all $X, Y \in \mathfrak{X}_{t}^{\bullet}(\mathcal{A})$. For braided differential forms $\omega=a_{0} \cdot \mathrm{d} a_{1} \wedge_{\mathcal{R}} \cdots \wedge_{\mathcal{R}} \mathrm{d} a_{n} \in \Omega_{\mathcal{R}}^{\bullet}(\mathcal{A})$ one defines

$$
\operatorname{pr}(\omega)=\operatorname{pr}\left(a_{0}\right) \mathrm{d}\left(\operatorname{pr}\left(a_{1}\right)\right) \wedge_{\mathcal{R}} \cdots \wedge_{\mathcal{R}} \mathrm{d}\left(\operatorname{pr}\left(a_{n}\right)\right),
$$

leading to a short exact sequence of differential graded algebras

$$
0 \rightarrow \operatorname{ker}(\mathrm{pr}) \rightarrow \Omega_{\mathcal{R}}^{\bullet}(\mathcal{A}) \stackrel{\mathrm{pr}}{\rightarrow} \Omega_{\mathcal{R}}^{\bullet}(\mathcal{A} / \mathcal{C}) \rightarrow 0,
$$

where $\operatorname{ker}(\mathrm{pr})=\bigoplus_{k \geq 0} \operatorname{ker}(\mathrm{pr})^{k}$ is defined recursively by $\operatorname{ker}(\mathrm{pr})^{0}=\mathcal{C}$ and

$$
\operatorname{ker}(\operatorname{pr})^{k+1}=\left\{\omega \in \Omega_{\mathcal{R}}^{k+1}(\mathcal{A}) \mid \mathbf{i}_{X}^{\mathcal{R}} \omega \in \operatorname{ker}(\operatorname{pr})^{k} \text { for all } X \in \mathfrak{X}_{t}^{1}(\mathcal{A})\right\}
$$

for $k \geq 0$. As in the case of $\mathfrak{X}_{\mathcal{R}}^{1}(\mathcal{A} / \mathcal{C})$, the projected actions, defined by intertwining the projections, structure $\Omega_{\mathcal{R}}^{\bullet}(\mathcal{A} / \mathcal{C})$ as an object in ${ }_{\mathcal{A} / \mathcal{C}}^{H} \mathcal{M}_{\mathcal{A} / \mathcal{C}}^{\mathcal{R}}$.

Theorem 4.2. The braided Cartan calculus on $\mathcal{A} / \mathcal{C}$ is the projection of the braided Cartan calculus on $\mathcal{A}$. Namely,

$$
\mathscr{L}_{\operatorname{pr}(X)}^{\mathcal{R}} \operatorname{pr}(\omega)=\operatorname{pr}\left(\mathscr{L}_{X}^{\mathcal{R}} \omega\right), \mathrm{i}_{\operatorname{pr}(X)}^{\mathcal{R}} \operatorname{pr}(\omega)=\operatorname{pr}\left(\mathrm{i}_{X}^{\mathcal{R}} \omega\right) \text { and } \mathrm{d}(\operatorname{pr}(\omega))=\operatorname{pr}(\mathrm{d} \omega)
$$

for all $X \in \mathfrak{X}_{t}^{\bullet}(\mathcal{A})$ and $\omega \in \Omega_{\mathcal{R}}^{\bullet}(\mathcal{A})$.

Proof. Equations (4.8) are easily verified on braided differential forms of order 0 and 1 . Since pr is a homomorphism of the braided wedge product the claim follows.

As a special case we recover that the Cartan calculus on a closed embedded submanifold $\iota: N \rightarrow M$ of a smooth manifold $M$ is obtained by the pullback $\iota^{*}: \Omega^{\bullet}(M) \rightarrow \Omega^{\bullet}(N)$ of differential forms and restriction $\iota^{*}: \mathfrak{X}_{t}^{\bullet}(M) \rightarrow \mathfrak{X}^{\bullet}(N)$ of tangent multivector fields to $N$. The latter is defined for any $X \in \mathfrak{X}_{t}^{1}(M)$ as the unique vector field $\left.X\right|_{N} \in \mathfrak{X}^{1}(N)$, which is $\iota$-related to $X$, i.e. $T_{q} \iota\left(\left.X\right|_{N}\right)_{q}=X_{\iota(q)}$ for all $q \in N$, where $T_{q} \iota: T_{q} N \rightarrow T_{\iota(q)} M$ denotes the tangent map (c.f. 30] Lemma 5.39). In particular,

$$
\mathscr{L}_{\iota^{*}(X)} \iota^{*}(\omega)=\iota^{*}\left(\mathscr{L}_{X} \omega\right), \quad \mathrm{i}_{\iota^{*}(X)} \iota^{*}(\omega)=\iota^{*}\left(\mathrm{i}_{X} \omega\right) \text { and } \mathrm{d} \iota^{*}(\omega)=\iota^{*}(\mathrm{~d} \omega)
$$

for all $X \in \mathfrak{X}^{\bullet}(M)$ and $\omega \in \Omega^{\bullet}(M)$.

In the next proposition we prove that the gauge equivalence given by the Drinfel'd functor is compatible with the notion of submanifold ideal, i.e. the projection to submanifold algebras and twisting commute. In the particular case of a cocommutative Hopf algebra with trivial triangular structure this means that twist quantization and projection to the submanifold algebra commute (see also [25]).

Proposition 4.3. For any twist $\mathcal{F}$ on $H$, the submanifold algebra projection of the twist deformation $\left(\mathfrak{X}_{t}^{\bullet}(\mathcal{A})_{\mathcal{F}}, \wedge_{\mathcal{F}}, \llbracket \cdot, \cdot \rrbracket_{\mathcal{F}}\right)$ of the braided Gerstenhaber algebra of tangent multivector fields on $\mathcal{A}$ coincides with the twist deformation $\left(\mathfrak{X}_{\mathcal{R}}^{\bullet}(\mathcal{A} / \mathcal{C})_{\mathcal{F}}, \wedge_{\mathcal{F}}, \llbracket \cdot, \rrbracket_{\mathcal{F}}\right)$ of the braided Gerstenhaber algebra of braided multivector fields on $\mathcal{A} / \mathcal{C}$. Moreover, the twisted Cartan calculus on $\mathcal{A} / \mathcal{C}$ is given by the projection of the twisted Cartan calculus on $\mathcal{A}$. Namely, $\Omega_{\mathcal{R}}^{\bullet}(\mathcal{A} / \mathcal{C})_{\mathcal{F}}=\operatorname{pr}\left(\Omega_{\mathcal{R}}^{\bullet}(\mathcal{A})_{\mathcal{F}}\right)$

$$
\mathscr{L}_{\operatorname{pr}(X)}^{\mathcal{F}} \operatorname{pr}(\omega)=\operatorname{pr}\left(\mathscr{L}_{X}^{\mathcal{F}} \omega\right), \mathrm{i}_{\operatorname{pr}(X)}^{\mathcal{F}} \operatorname{pr}(\omega)=\operatorname{pr}\left(\mathrm{i}_{X}^{\mathcal{F}} \omega\right) \text { and } \mathrm{d}(\operatorname{pr}(\omega))=\operatorname{pr}(\mathrm{d} \omega)
$$

for all $X \in \mathfrak{X}_{t}^{\bullet}(\mathcal{A})_{\mathcal{F}}$ and $\omega \in \Omega_{\mathcal{R}}^{\bullet}(\mathcal{A})_{\mathcal{F}}$.

Proof. Note that the twist deformation of $\mathfrak{X}_{t}^{\bullet}(\mathcal{A})$ is a braided Gerstenhaber algebra since the braided multivector fields which are tangent to $\mathcal{C}$ are an $H$-submodule and a braided symmetric $\mathcal{A}$-sub-bimodule of $\mathfrak{X}_{\mathcal{R}}^{\bullet}(\mathcal{A})$. We already noticed that pr: $\mathfrak{X}_{t}^{\bullet}(\mathcal{A}) \rightarrow \mathfrak{X}_{\mathcal{R}}^{\bullet}(\mathcal{A} / \mathcal{C})$ is surjective. Let $X, Y \in \mathfrak{X}_{t}^{\bullet}(\mathcal{A})_{\mathcal{F}}$ and $a \in \mathcal{A}$. Then

$$
\operatorname{pr}(X) \wedge_{\mathcal{F}} \operatorname{pr}(Y)=\left(\mathcal{F}_{1}^{-1} \triangleright \operatorname{pr}(X)\right) \wedge_{\mathcal{R}}\left(\mathcal{F}_{2}^{-1} \triangleright \operatorname{pr}(Y)\right)=\operatorname{pr}\left(X \wedge_{\mathcal{F}} Y\right),
$$

and similarly $\llbracket \operatorname{pr}(X), \operatorname{pr}(Y) \rrbracket_{\mathcal{F}}=\operatorname{pr}\left(\llbracket X, Y \rrbracket_{\mathcal{F}}\right)$ and $\operatorname{pr}(a) \cdot_{\mathcal{F}} \operatorname{pr}(X)=\operatorname{pr}\left(a \cdot_{\mathcal{F}} X\right)$ follow. Moreover,

and

$$
\mathscr{L}_{\operatorname{pr}(X)}^{\mathcal{F}} \operatorname{pr}(\omega)=\mathscr{L}_{\mathcal{F}_{1}^{-1} \triangleright \operatorname{pr}(X)}^{\mathcal{R}}\left(\mathcal{F}_{2}^{-1} \triangleright \operatorname{pr}(\omega)\right)=\operatorname{pr}\left(\mathscr{L}_{X}^{\mathcal{F}} \omega\right)
$$

$$
\mathrm{i}_{\operatorname{pr}(X)}^{\mathcal{F}} \operatorname{pr}(\omega)=\mathrm{i}_{\mathcal{F}_{1}^{-1} \triangleright \operatorname{pr}(X)}^{\mathcal{R}}\left(\mathcal{F}_{2}^{-1} \triangleright \operatorname{pr}(\omega)\right)=\operatorname{pr}\left(\mathrm{i}_{X}^{\mathcal{F}} \omega\right)
$$

for all $X \in \mathfrak{X}_{t}^{\bullet}(\mathcal{A})$ and $\omega \in \Omega_{\mathcal{R}}^{\bullet}(\mathcal{A})$ by Theorem 4.2 . This concludes the proof of the proposition. 


\subsection{Equivariant Covariant Derivatives on Submanifolds}

In this section we discuss equivariant covariant derivatives on submanifold algebras and study under which conditions equivariant covariant derivatives and metrics allow for projections. Accepting two mild axioms the latter is possible for a given strongly non-degenerate equivariant metric. Furthermore, the projection of the equivariant covariant derivative is compatible with the notion of curvature, torsion and twist deformation.

Fix a submanifold ideal $\mathcal{C}$ of $\mathcal{A}$ and a strongly non-degenerate equivariant metric $\mathrm{g}$ on $\mathcal{A}$. Then there is a direct sum decomposition

$$
\mathfrak{X}_{\mathcal{R}}^{1}(\mathcal{A})=\mathfrak{X}_{t}^{1}(\mathcal{A}) \oplus \mathfrak{X}_{n}^{1}(\mathcal{A}),
$$

where $\mathfrak{X}_{n}^{1}(\mathcal{A})$ are the so-called braided normal vector fields with respect to $\mathcal{C}$ and $\mathbf{g}$, defined to be the subspace orthogonal to $\mathfrak{X}_{t}^{1}(\mathcal{A})$ with respect to $\mathrm{g}$. Then, $\operatorname{pr}_{\mathbf{g}}: \mathfrak{X}_{\mathcal{R}}^{1}(\mathcal{A}) \rightarrow \mathfrak{X}_{\mathcal{R}}^{1}(\mathcal{A} / \mathcal{C})$ is the $\mathbb{k}$-linear map which first projects to the first addend in the above decomposition and applies pr: $\mathfrak{X}_{t}^{1}(\mathcal{A}) \rightarrow \mathfrak{X}_{\mathcal{R}}^{1}(\mathcal{A} / \mathcal{C})$ afterwards. In particular $\operatorname{pr}_{\mathbf{g}}(X)=\operatorname{pr}(X)$ for all $X \in \mathfrak{X}_{t}^{1}(\mathcal{A})$. In a next step we define a $\mathbb{k}$-linear map $\mathbf{g}_{\mathcal{A} / \mathcal{C}}: \mathfrak{X}_{\mathcal{R}}^{1}(\mathcal{A} / \mathcal{C}) \otimes_{\mathcal{A} / \mathcal{C}} \mathfrak{X}_{\mathcal{R}}^{1}(\mathcal{A} / \mathcal{C}) \rightarrow \mathcal{A} / \mathcal{C}$ by

$$
\mathbf{g}_{\mathcal{A} / \mathcal{C}}\left(\operatorname{pr}_{\mathbf{g}}(X), \operatorname{pr}_{\mathbf{g}}(Y)\right)=\operatorname{pr}_{\mathbf{g}}(\mathbf{g}(X, Y))
$$

for all $X, Y \in \mathfrak{X}_{\mathcal{R}}^{1}(\mathcal{A})$. It is well-defined if $\mathfrak{X}_{0}^{1}(\mathcal{A})=$ ker pr has the following property.

Axiom 1: for every $X \in \mathfrak{X}_{0}^{1}(\mathcal{A})$ there are finitely many $c_{i} \in \mathcal{C}$ and $X^{i} \in \mathfrak{X}_{t}^{1}(\mathcal{A})$ such that $X=\sum_{i} c_{i} X^{i}$.

This is for example the case if $\mathfrak{X}_{0}^{1}(\mathcal{A})$ is finitely generated as a $\mathcal{C}$-bimodule. If $\mathbf{g}$ is nondegenerate the projection $\mathbf{g}_{\mathcal{A} / \mathcal{C}}$ is not non-degenerate in general. However, if we assume the following property of $\mathbf{g}$, the projection $\mathbf{g}_{\mathcal{A} / \mathcal{C}}$ is strongly non-degenerate if $\mathbf{g}$ is.

Axiom 2: if $X \in \mathfrak{X}_{t}^{1}(\mathcal{A})$, then $\mathbf{g}(X, X) \in \mathcal{C}$ implies $X \in \mathfrak{X}_{0}^{1}(\mathcal{A})$.

Note that in the case of closed embedded smooth manifolds both axiom 1 and 2 are satisfied.

Proposition 4.4. For any strongly non-degenerate equivariant metric $\mathbf{g}$ on $\mathcal{A}$ such that the axioms 1 and 2 are satisfied, $\mathbf{g}_{\mathcal{A} / \mathcal{C}}$ is a well-defined strongly non-degenerate equivariant metric on $\mathcal{A} / \mathcal{C}$. The projection

$$
\nabla_{\operatorname{pr}(X)}^{\mathcal{A} / \mathcal{C}} \operatorname{pr}(Y)=\operatorname{pr}_{\mathbf{g}}\left(\nabla_{X}^{\mathcal{R}} Y\right)
$$

of an equivariant covariant derivative $\nabla^{\mathcal{R}}: \mathfrak{X}_{\mathcal{R}}^{1}(\mathcal{A}) \otimes \mathfrak{X}_{\mathcal{R}}^{1}(\mathcal{A}) \rightarrow \mathfrak{X}_{\mathcal{R}}^{1}(\mathcal{A})$ on $\mathcal{A}$, where $X, Y \in$ $\mathfrak{X}_{t}^{1}(\mathcal{A})$, is an equivariant covariant derivative with respect to $\mathcal{R}$ on $\mathcal{A} / \mathcal{C}$. If furthermore, $\nabla^{\mathcal{R}}$ is the equivariant Levi-Civita covariant derivative with respect to $\mathrm{g}, \nabla^{\mathcal{A} / \mathcal{C}}$ is the equivariant Levi-Civita covariant derivative on $\mathcal{A} / \mathcal{C}$ with respect to $\mathbf{g}_{\mathcal{A} / \mathcal{C}}$.

Proof. Axiom 1 assures $\mathbf{g}_{\mathcal{A} / \mathcal{C}}$ to be well-defined, since

$$
\begin{aligned}
\mathbf{g}_{\mathcal{A} / \mathcal{C}}\left(\operatorname{pr}_{\mathbf{g}}(X), \operatorname{pr}_{\mathbf{g}}(Y)\right) & =\mathbf{g}_{\mathcal{A} / \mathcal{C}}\left(\operatorname{pr}_{\mathbf{g}}\left(\sum_{i} c_{i} \cdot X^{i}\right), \operatorname{pr}_{\mathbf{g}}(Y)\right)=\operatorname{pr}\left(\mathbf{g}\left(\sum_{i} c_{i} \cdot X^{i}, Y\right)\right) \\
& =\operatorname{pr}(\underbrace{\sum_{i} c_{i} \cdot \mathbf{g}\left(X^{i}, Y\right)}_{\in \mathcal{C}})=0
\end{aligned}
$$

and similarly $\mathbf{g}_{\mathcal{A} / \mathcal{C}}\left(\operatorname{pr}_{\mathbf{g}}(Y), \operatorname{pr}_{\mathbf{g}}(X)\right)=0$ for all $X \in \mathfrak{X}_{0}^{1}(\mathcal{A})$ and $Y \in \mathfrak{X}_{\mathcal{R}}^{1}(\mathcal{A})$. Let $X \in \mathfrak{X}_{\mathcal{R}}^{1}(\mathcal{A} / \mathcal{C})$ and choose $Y \in \mathfrak{X}_{t}^{1}(\mathcal{A})$ such that $\operatorname{pr}(Y)=X$. Then

$$
0=\mathbf{g}_{\mathcal{A} / \mathcal{C}}(X, X)=\operatorname{pr}(\mathbf{g}(Y, Y))
$$

implies $\mathbf{g}(Y, Y) \in \mathcal{C}$, i.e. $Y \in \mathfrak{X}_{0}^{1}(\mathcal{A})$ by Axiom 2. In other words $\mathbf{g}_{\mathcal{A} / \mathcal{C}}(X, X)=0$ implies $X=0$, which is equivalent to the statement that $X \neq 0 \operatorname{implies} \mathbf{g}_{\mathcal{A} / \mathcal{C}}(X, X) \neq 0$, i.e. strong non-degeneracy of $\mathbf{g}_{\mathcal{A} / \mathcal{C}}$. From Axiom 1 it follows that $\nabla^{\mathcal{A} / \mathcal{C}}$ is well-defined. In fact, for $X=\sum_{i} c_{i} \cdot X^{i} \in \mathfrak{X}_{0}^{1}(\mathcal{A})$ and $Y \in \mathfrak{X}_{t}^{1}(\mathcal{A})$ we obtain

$$
\nabla_{\operatorname{pr}(X)}^{\mathcal{A} / \mathcal{C}} \operatorname{pr}(Y)=\operatorname{pr}_{\mathbf{g}}\left(\nabla_{X}^{\mathcal{R}} Y\right)=\operatorname{pr}_{\mathbf{g}}(\underbrace{\sum_{i} c_{i} \cdot \nabla_{X^{i}}^{\mathcal{R}} Y}_{\in \mathfrak{X}_{0}^{1}(\mathcal{A})})=0
$$


and

$$
\begin{aligned}
& \nabla_{\operatorname{pr}(Y)}^{\mathcal{A} / \mathcal{C}} \operatorname{pr}(X)=\operatorname{pr}_{\mathbf{g}}\left(\sum_{i} \nabla_{Y}^{\mathcal{R}}\left(c_{i} \cdot X^{i}\right)\right)
\end{aligned}
$$

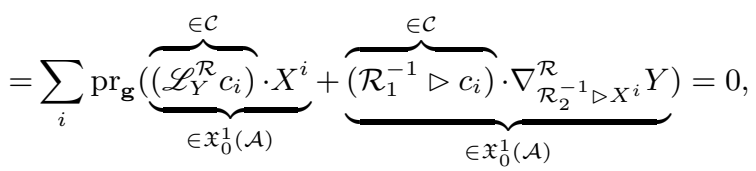

since $\nabla^{\mathcal{R}}$ is left $\mathcal{A}$-linear in the first argument and satisfies a braided Leibniz rule in the second argument. The remaining results are proven in 41 .

We would like to stress that the assumptions of Proposition 4.4 are sufficient to project strongly non-degenerate equivariant metrics and equivariant covariant derivatives to submanifold algebras. Whether those conditions are also necessary is part of further investigation. Fix an equivariant covariant derivative $\nabla^{\mathcal{R}}$ on $\mathcal{A}$ and a strongly non-degenerate equivariant metric $\mathbf{g}$ such that axiom 1 and 2 hold. The curvature and torsion of a projected equivariant covariant derivative coincide with the projection of the curvature and torsion of $\nabla^{\mathcal{R}}$.

Corollary 4.5. The curvature $R^{\nabla^{\mathcal{A} / \mathcal{C}}}$ and the torsion $\operatorname{Tor}^{\nabla^{\mathcal{A} / \mathcal{C}}}$ of the projected equivariant covariant derivative $\nabla^{\mathcal{A} / \mathcal{C}}$ are given by

$$
R^{\nabla^{\mathcal{A} / \mathcal{C}}}(\operatorname{pr}(X), \operatorname{pr}(Y))(\operatorname{pr}(Z))=\operatorname{pr}_{\mathbf{g}}\left(R^{\nabla^{\mathcal{R}}}(X, Y) Z\right)
$$

and

for all $X, Y, Z \in \mathfrak{X}_{t}^{1}(\mathcal{A})$.

$$
\operatorname{Tor}^{\nabla^{\mathcal{A} / \mathcal{C}}}(\operatorname{pr}(X), \operatorname{pr}(Y))=\operatorname{pr}_{\mathbf{g}}\left(\operatorname{Tor}^{\nabla^{\mathcal{R}}}(X, Y)\right)
$$

One extends the projection $\operatorname{pr}_{\mathrm{g}}: \mathfrak{X}_{\mathcal{R}}^{\bullet}(\mathcal{A}) \rightarrow \mathfrak{X}_{\mathcal{R}}^{\bullet}(\mathcal{A} / \mathcal{R})$ to braided multivector fields by defining it to coincide with pr on $\mathcal{A}$ and to be a homomorphism of the braided wedge product on higher wedge powers. On braided differential forms we set $\operatorname{pr}_{\mathbf{g}}=\mathrm{pr}$.

Corollary 4.6. The equivariant covariant derivatives

$$
\nabla^{\mathcal{A} / \mathcal{C}}: \mathfrak{X}_{\mathcal{R}}^{1}(\mathcal{A} / \mathcal{C}) \otimes \mathfrak{X}_{\mathcal{R}}^{\bullet}(\mathcal{A} / \mathcal{C}) \rightarrow \mathfrak{X}_{\mathcal{R}}^{\bullet}(\mathcal{A} / \mathcal{C}) \text { and } \tilde{\nabla}^{\mathcal{A} / \mathcal{C}}: \mathfrak{X}_{\mathcal{R}}^{1}(\mathcal{A} / \mathcal{C}) \otimes \Omega_{\mathcal{R}}^{\bullet}(\mathcal{A} / \mathcal{C}) \rightarrow \Omega_{\mathcal{R}}^{\bullet}(\mathcal{A} / \mathcal{C}),
$$

induced by the projected equivariant covariant derivative $\nabla^{\mathcal{A} / \mathcal{C}}$ on $\mathcal{A} / \mathcal{C}$ according to Proposition 3.10 , are projected from the covariant derivatives induced by $\nabla^{\mathcal{R}}$. Namely,

$$
\nabla_{\operatorname{pr}(X)}^{\mathcal{A} / \mathcal{C}} \operatorname{pr}(Y)=\operatorname{pr}_{\mathbf{g}}\left(\nabla_{X}^{\mathcal{R}} Y\right) \text { and } \tilde{\nabla}_{\operatorname{pr}(X)}^{\mathcal{A} / \mathcal{C}} \operatorname{pr}(\omega)=\operatorname{pr}_{\mathbf{g}}\left(\tilde{\nabla}_{X}^{\mathcal{R}} \omega\right)
$$

for all $X \in \mathfrak{X}_{t}^{1}(\mathcal{A}), Y \in \mathfrak{X}_{t}^{\bullet}(\mathcal{A})$ and $\omega \in \Omega_{\mathcal{R}}^{\bullet}(\mathcal{A})$.

Furthermore, twisted equivariant covariant derivatives behave well under projection.

Proposition 4.7. For any twist $\mathcal{F}$ on $H$, the projection of the twisted equivariant covariant derivative coincides with the twist deformation of the projected equivariant covariant derivative, i.e. $\left(\nabla^{\mathcal{A} / \mathcal{C}}\right)_{\operatorname{pr}(X)}^{\mathcal{F}} \operatorname{pr}(Y)=\operatorname{pr}_{\mathbf{g}}\left(\nabla_{X}^{\mathcal{F}} Y\right)$ for all $X, Y \in \mathfrak{X}_{t}^{1}(\mathcal{A})_{\mathcal{F}}$. Its curvature and torsion are given by

and

$$
\begin{aligned}
R^{\left(\nabla^{\mathcal{A} / \mathcal{C}}\right)^{\mathcal{F}}}(\operatorname{pr}(X), \operatorname{pr}(Y))(\operatorname{pr}(Z)) \\
=R^{\nabla^{\mathcal{A} / \mathcal{C}}}\left(\left(\mathcal{F}_{1(1)}^{-1} \mathcal{F}_{1}^{\prime}-1\right) \triangleright \operatorname{pr}(X),\left(\mathcal{F}_{1(2)}^{-1} \mathcal{F}_{2}^{\prime}-1\right) \triangleright \operatorname{pr}(Y)\right)\left(\mathcal{F}_{2}^{-1} \triangleright \operatorname{pr}(Z)\right) \\
=\operatorname{pr}\left(R^{\nabla^{\mathcal{R}}}\left(\left(\mathcal{F}_{1(1)}^{-1} \mathcal{F}_{1}^{\prime}-1\right) \triangleright X,\left(\mathcal{F}_{1(2)}^{-1} \mathcal{F}_{2}^{\prime}-1\right) \triangleright Y\right)\left(\mathcal{F}_{2}^{-1} \triangleright Z\right)\right)
\end{aligned}
$$

$$
\begin{aligned}
& \operatorname{Tor}^{\left(\nabla^{\mathcal{A} / \mathcal{C}}\right)^{\mathcal{F}}}(\operatorname{pr}(X), \operatorname{pr}(Y))=\operatorname{Tor}^{\nabla^{\mathcal{A} / \mathcal{C}}}\left(\mathcal{F}_{1}^{-1} \triangleright \operatorname{pr}(X), \mathcal{F}_{2}^{-1} \triangleright \operatorname{pr}(Y)\right) \\
& =\operatorname{pr}\left(\operatorname{Tor}^{\nabla^{\mathcal{R}}}\left(\mathcal{F}_{1}^{-1} \triangleright X, \mathcal{F}_{2}^{-1} \triangleright Y\right)\right)
\end{aligned}
$$

for all $X, Y, Z \in \mathfrak{X}_{t}^{1}(\mathcal{A})_{\mathcal{F}}$, respectively. Similar statements hold for the induced (twisted) equivariant covariant derivatives on braided differential forms and braided multivector fields.

Proof. For all $X, Y \in \mathfrak{X}_{t}^{1}(\mathcal{A})_{\mathcal{F}}$ one obtains

$$
\begin{aligned}
& \operatorname{pr}_{\mathbf{g}}\left(\nabla_{X}^{\mathcal{F}} Y\right)=\operatorname{pr}_{\mathbf{g}}\left(\nabla_{\mathcal{F}_{1}^{-1} \triangleright X}^{\mathcal{R}}\left(\mathcal{F}_{2}^{-1} \triangleright Y\right)\right)=\nabla_{\operatorname{pr}\left(\mathcal{F}_{1}^{-1} \triangleright X\right)}^{\mathcal{A} / \mathcal{C}}\left(\operatorname{pr}\left(\mathcal{F}_{2}^{-1} \triangleright Y\right)\right) \\
& =\nabla_{\mathcal{F}_{1}^{-1} \triangleright \operatorname{pr}(X)}^{\mathcal{A} / \mathcal{C}}\left(\mathcal{F}_{2}^{-1} \triangleright \operatorname{pr}(Y)\right)=\left(\nabla^{\mathcal{A} / \mathcal{C}}\right)_{\operatorname{pr}(X)}^{\mathcal{F}} \operatorname{pr}(Y)
\end{aligned}
$$

and similarly one proves the statements about the induced equivariant covariant derivatives. 


\section{Acknowledgments}

The author is grateful to Francesco D'Andrea and Gaetano Fiore for their constant support. In particular, he wants to thank the latter for introducing him to the concept of twisted Cartan calculus and proposing to prove compatibility with projections to submanifold algebras. Special thanks go to Paolo Aschieri and the referee for their valuable comments on the first version of this paper. Furthermore, the author wants to thank Stefan Waldmann for posing a question at the DQ seminar about the existence of a braided Cartan calculus for every triangular structure.

\section{References}

[1] Arnlind, J. And Norkvist, A. T.: Noncommutative minimal embeddings and morphisms of pseudo-Riemannian calculi. Preprint arXiv:1906.03885, 2019.

[2] Aschieri, P. And Castellani, L.: Noncommutative gravity solutions. J. Geom. Phys., 60(3):375-393, 2010.

[3] Aschieri, P. And Lizzi, F. And Vitale, P.: Twisting all the way: from Classical Mechanics to Quantum Fields. Phys. Rev. D77, 2008.

[4] Aschieri, P. And Schenkel, A.: Noncommutative connections on bimodules and Drinfeld twist deformation. Adv. Theor. Math. Phys., 18(3):513-612, 2014.

[5] Aschieri, P. And Dimitrijevic, M. And Meyer, F. And Wess, J.: Noncommutative geometry and gravity. Class. Quantum Gravity, 23:1883-1911, 2006.

[6] Barnes, G. E. And Schenkel, A. And Szabo, R. J.: Nonassociative geometry in quasiHopf representation categories I: Bimodules and their internal homomorphisms. J. Geom. Phys., 89:111-152, 2015.

[7] Barnes, G. E. And Schenkel, A. And Szabo, R. J.: Nonassociative geometry in quasiHopf representation categories II: Connections and curvature, J. Geom. Phys., 106:234-255, 2016.

[8] Bayen, F. and Flato, M. and Fronsdal, C. and Lichnerowicz, A. and SternHEIMER, D.: Deformation theory and quantization. I. Deformations of symplectic structures. Ann. Phys., 111(1):61-110, 1978.

[9] Bhowmick, J. And Goswami, D. And Landi, G.: On the Koszul formula in noncommutative geometry. Preprint arXiv:1910.09306, 2019.

[10] Berenstein, A. And Zwicknagl, S.: Braided Symmetric and Exterior Algebras. Trans. Amer. Math. Soc., 360(7):3429-3472, 2008.

[11] Bieliavsky, P. and Esposito, C. and Waldmann, S. and Weber, T.: Obstructions for twist star products. Lett. Math. Phys., 108(5):1341-1350, 2018.

[12] Chari, V. And Pressley, A.: A Guide to Quantum Groups. Cambridge University Press, 1994.

[13] Connes, A.: Noncommutative Geometry. Academic Press, 1995.

[14] D'Andrea, F.: Submanifold Algebras. Preprint arXiv:1912.01225, 2019.

[15] D'Andrea, F. And Weber, T.: Twist star products and Morita equivalence. C. R. Acad. Sci. Paris, 355(11):1178-1184, 2017.

[16] Drinfel'D, V. G.: Hopf algebras and the quantum Yang-Baxter equation. Soviet Math. Dokl., 28(3):667-671, 1983.

[17] Drinfel'D, V. G.: Quasi Hopf algebras. Alg. Anal., 1989.

[18] Dubois-Violette, M. And Michor, P. W.: Connections on central bimodules in noncommutative differential geometry. J. Geom. Phys., 20(2-3):218-232, 1996.

[19] Dubois-Violette, M. And Michor, P. W.: The Frolicher-Nijenhuis bracket for derivation based noncommutative differential forms. LPTHE-ORSAY-94-41, ESI-133-1994.

[20] Esposito, C. And Schnitzer, J. And Waldmann, S.: A universal construction of universal deformation formulas, Drinfeld twists and their positivity. Pac. J. Math., 291(2):319-358, 2017.

[21] Etingof, P. And Schiffmann, O.: Lectures on Quantum Groups. Lectures in mathematical physics, International Press, 2010. 
[22] Fiore, G.: Drinfeld twist and $q$ - deforming maps for Lie group covariant Heisenberg algebrae. Rev. Math. Phys., 12:327-359, 2000.

[23] Fiore, G.: On second quantization on noncommutative spaces with twisted symmetries. J. Phys. A, 43(15), 2010.

[24] Fiore, G. And Pisacane, F.: Fuzzy circle and new fuzzy sphere through confining potentials and energy cutoffs. J. Geom. Phys., 132:423-451, 2018.

[25] Fiore, G. and Weber, T.: Twisted Submanifolds of $\mathbb{R}^{n}$. In preparation, 2020.

[26] Giaquinto, A. And Zhang, J. J.: Bialgebra actions, twists, and universal deformation formulas. J. Pure Appl. Algebra, 128(2):133-151, 1998.

[27] Huebschmann, J.: Lie-Rinehart algebras, Gerstenhaber algebras and Batalin-Vilkovisky algebras. Ann. Inst. Fourier, 48(2):425-440, 1998.

[28] Kassel, C.: Quantum Groups. Springer Science+Buiseness Media New York, 1995.

[29] Lazzarini, S. And Masson, T.: Connections on Lie algebroids and on derivation-based noncommutative geometry. J. Geom. Phys., 62:387-402, 2012.

[30] LeE, J. M.: Introduction to Smooth Manifolds. Graduate Texts in Mathematics, 218, Springer-Verlag New York, 2003.

[31] MaJid, S.: Foundations of Quantum Group Theory. Cambridge University Press, 1995.

[32] Masson, T.: Submanifolds and Quotient Manifolds in Noncommutative Geometry. J. Math. Phys., 37(5), 1996.

[33] Meljanac, S. And Meljanac, D. And Pachol, A. And Pikutić, D.: Remarks on simple interpolation between Jordanian twists. J. Phys. A: Math. Theor., 50, 2017.

[34] Montgomery, S.: Hopf Algebras and Their Actions on Rings. CBMS Reg. Conf. Ser. Math., 82, 1993.

[35] Peterka, M. A. And Jeu-Liang Sheu, A.: On noncommutative Levi-Civita connections. Int. J. Geom. Methods Mod. Phys., 14(5), 2017.

[36] Schupp, P.: Cartan calculus: Differential geometry for quantum groups. Como Quantum Groups, 507-524, 1994.

[37] Schupp, P.: From quantum planes to quantum groups and back: Cartan calculus. arXiv eprint hep-th/9312076, 1993.

[38] Tamarkin, D. And Tsygan, B. L.: Noncommutative differential calculus, homotopy BV algebras and formality conjectures. Methods Funct. Anal. Topology, 6(2):85-100, 2000.

[39] Tsygan, B. L.: Noncommutative Calculus and Operads. Topics in Noncommutative Geometry, Clay Mathematics Proceedings, 16:19-66, 2012.

[40] Waldmann, S.: Recent Developments in Deformation Quantization. Quantum Mathematical Physics, 421-439, 2016.

[41] Weber, T.: Braided Commutative Geometry and Drinfel'd Twist Deformations. PhD Thesis, 2019

[42] Woronowicz, S. L.: Differential calculus on compact matrix pseudogroups (quantum groups). Comm. Math. Phys., 122(1):125-170, 1989. 